\title{
A Silyl-Nickel Moiety as a Metal-Ligand Cooperative Site
}

\author{
Jin Kim, Yeong-Eun Kim, Koeun Park and Yunho Lee* \\ Department of Chemistry, Korea Advanced Institute of Science and Technology, \\ Daejeon 34141, Republic of Korea
}

\section{Contents}

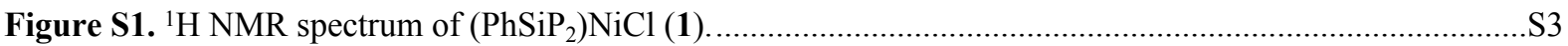

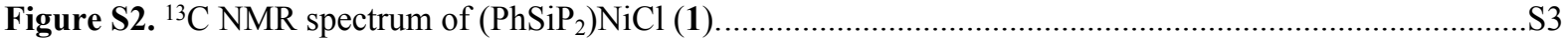

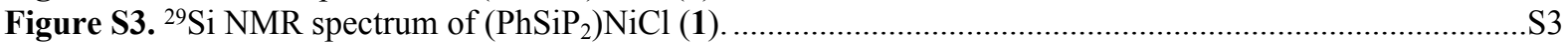

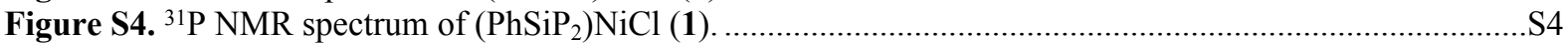

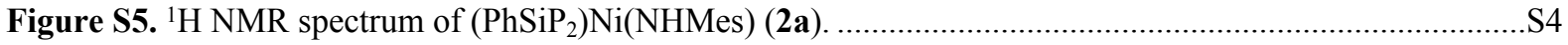

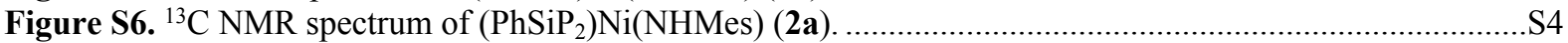

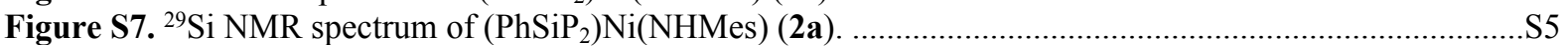

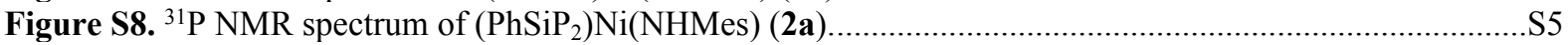

Figure S9. ${ }^{31} \mathrm{P}$ NMR spectrum of in situ reaction mixture of 1, 2,4,6-triisopropylaniline and NaHMDS...........S5

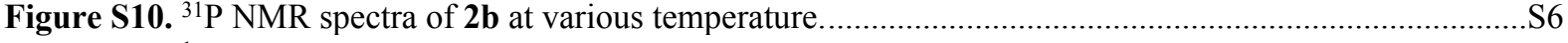

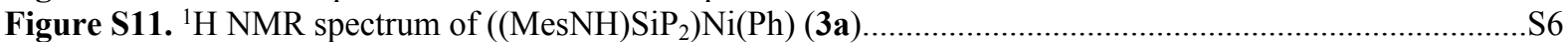

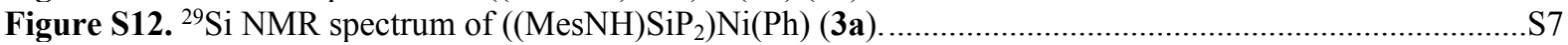

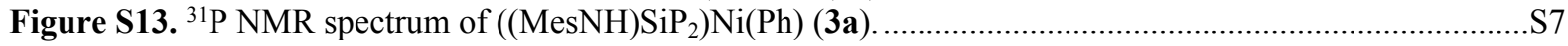

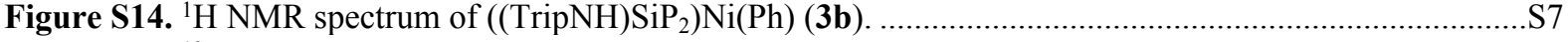

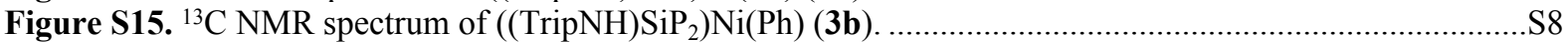

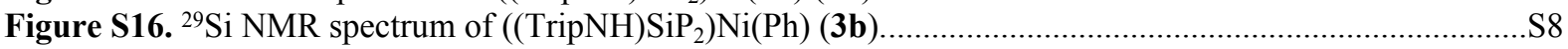

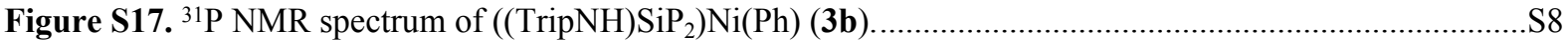

Figure S18. ${ }^{31} \mathrm{P}$ NMR spectrum of an unknown species, obtained from reaction of $\mathbf{2 a}$ and CO.......................S9

Figure S19. ${ }^{31} \mathrm{P}$ NMR spectrum of an unknown species, obtained from reaction of $\mathbf{2 b}$ and CO......................S9

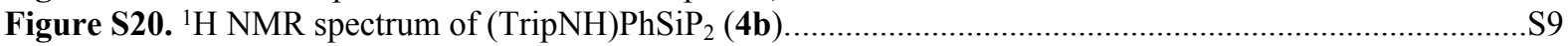

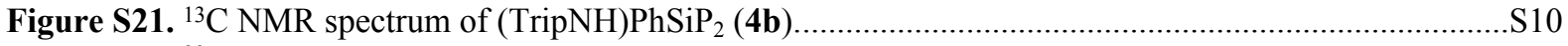

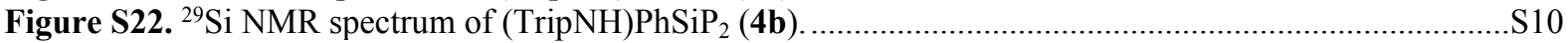

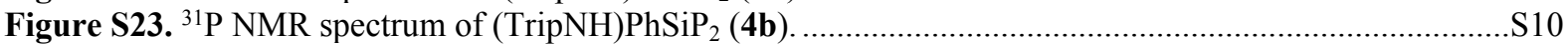

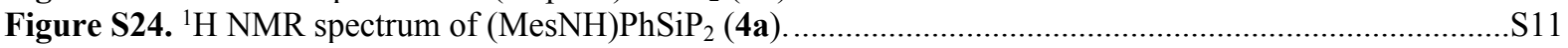

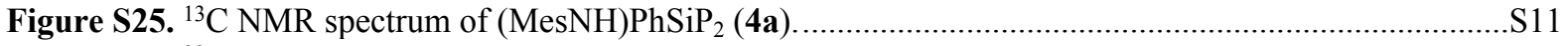

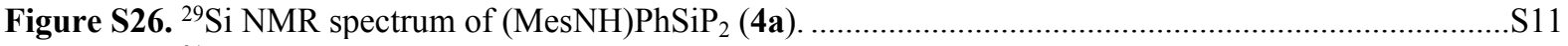

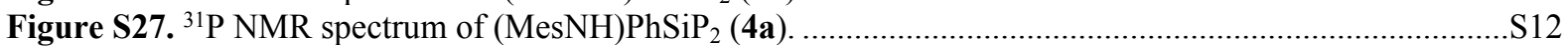

Figure S28. ${ }^{31} \mathrm{P}$ NMR spectrum of in situ reaction mixture of 3a and tert-butyl isocyanide...........................S12

Figure S29. ${ }^{31} \mathrm{P}$ NMR spectrum of in situ reaction mixture of $\mathbf{3 b}$ and tert-butyl isocyanide...........................S12

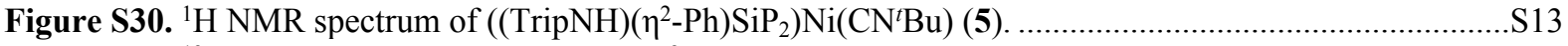

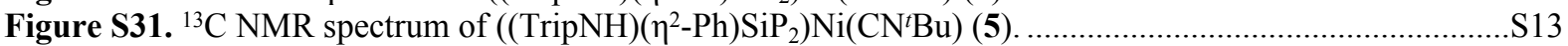

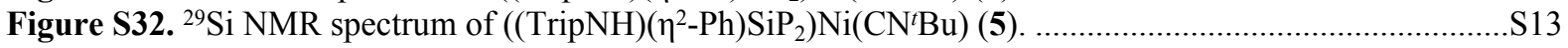

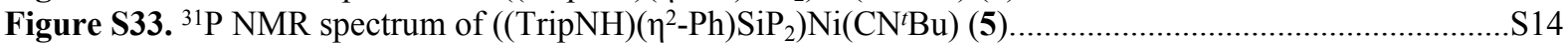

Figure S34. ${ }^{31} \mathrm{P}$ NMR spectra of the reaction of $\mathbf{2 a}$ with 0.1 equivalent of isocyanides...............................S14

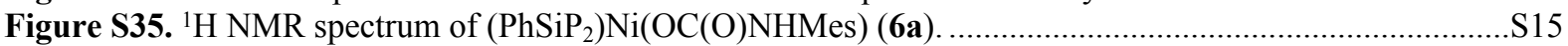

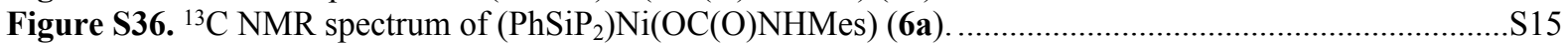

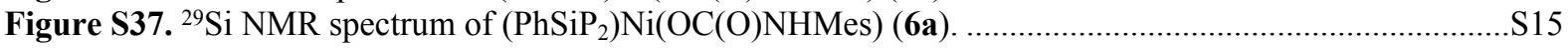

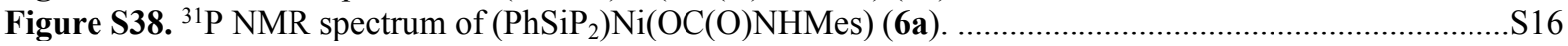

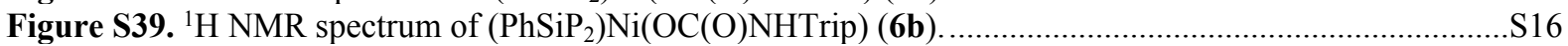

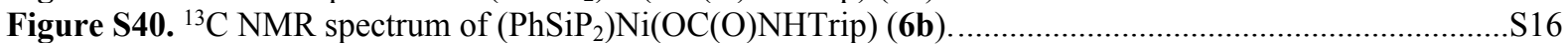

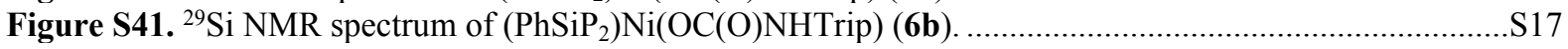

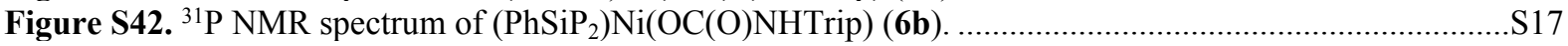

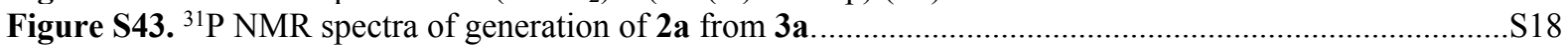

Figure S44. ${ }^{31} \mathrm{P}$ NMR spectra of generation of 2a and 6a from 3a under $\mathrm{N}_{2}$ and $\mathrm{CO}_{2}$ atmosphere.................S18

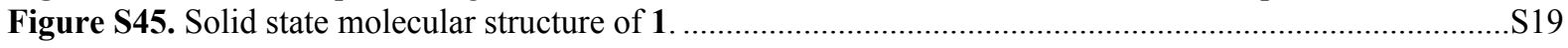




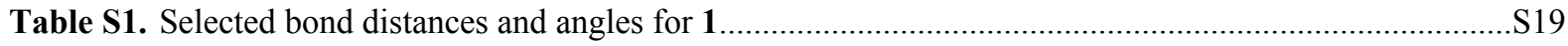

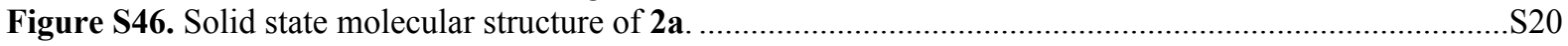

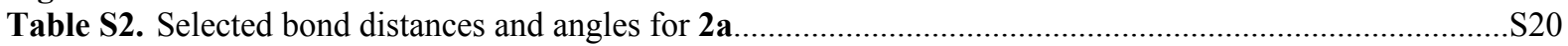

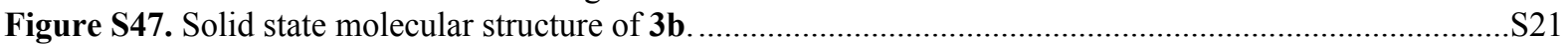

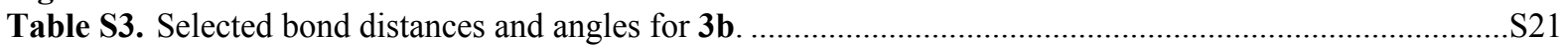

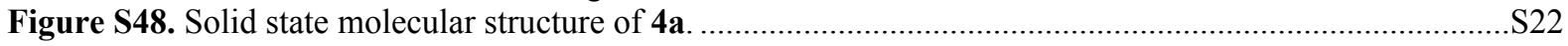

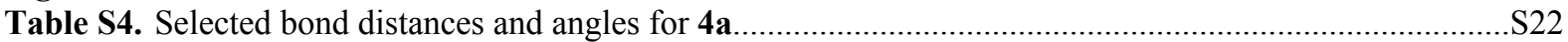

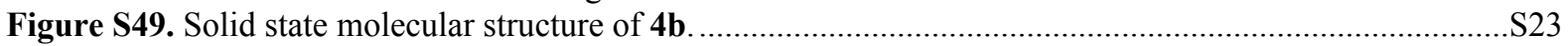

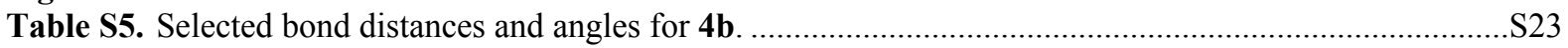

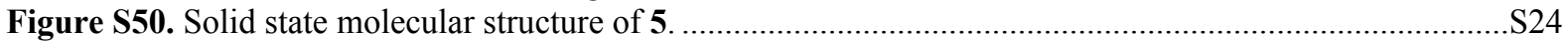

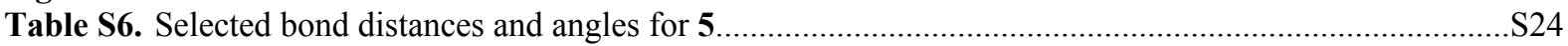

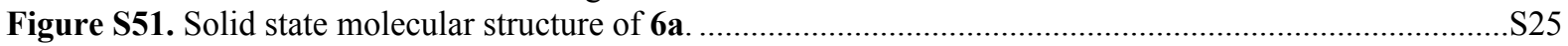

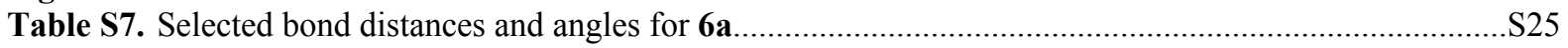

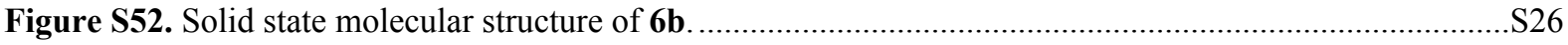

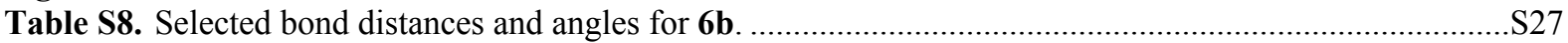

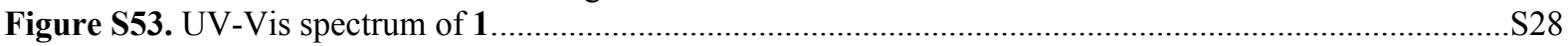

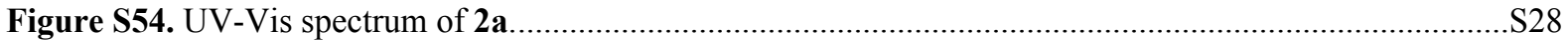

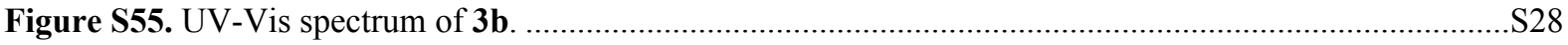

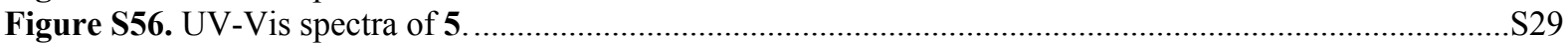

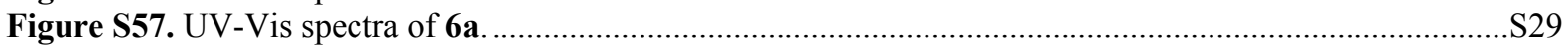

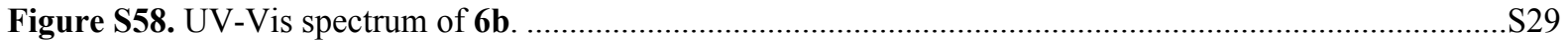

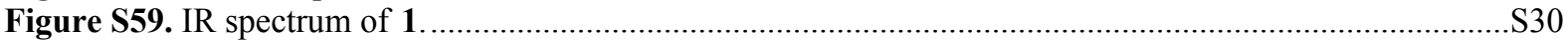

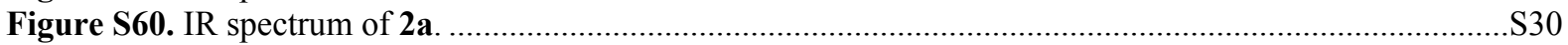

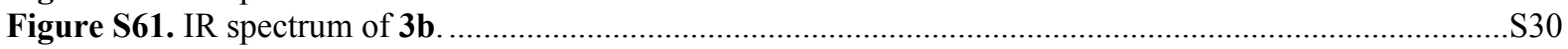

Figure S62. IR spectrum of an unknown species, obtained from reaction of $\mathbf{2 b}$ and CO.............................S31

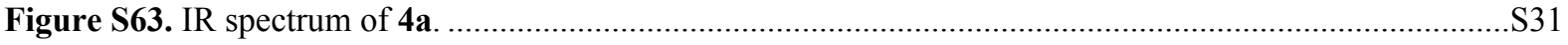

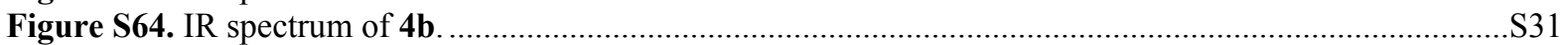

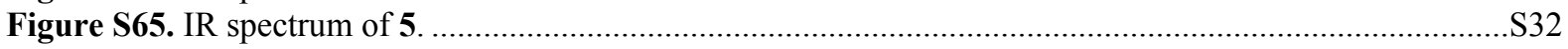

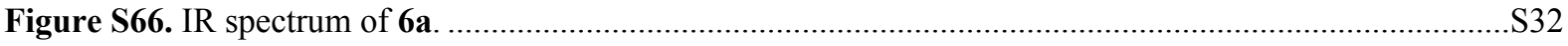

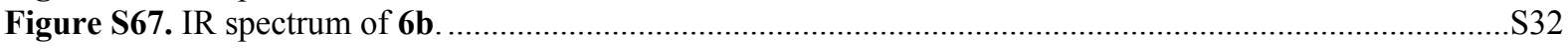

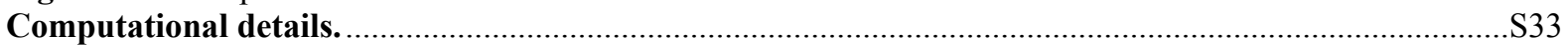

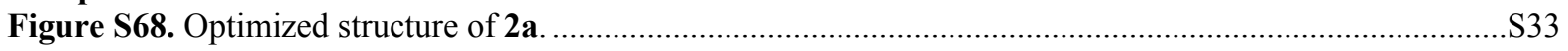

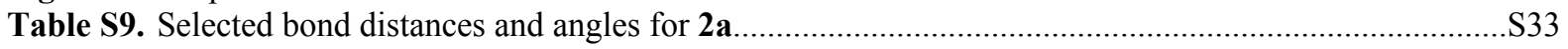

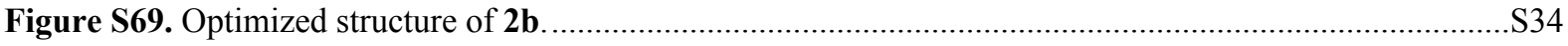

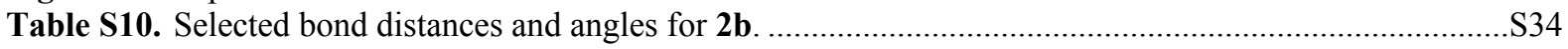

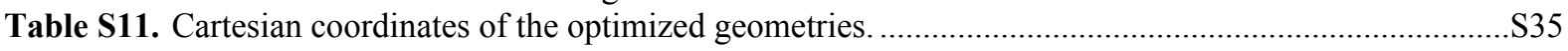

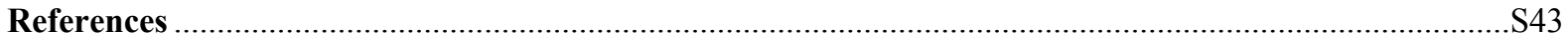


Figure S1. ${ }^{1} \mathrm{H}$ NMR spectrum $\left(\mathrm{C}_{6} \mathrm{D}_{6}, 400 \mathrm{MHz}\right)$ of $\left(\mathrm{PhSiP}_{2}\right) \mathrm{NiCl}(\mathbf{1})$ at room temperature.

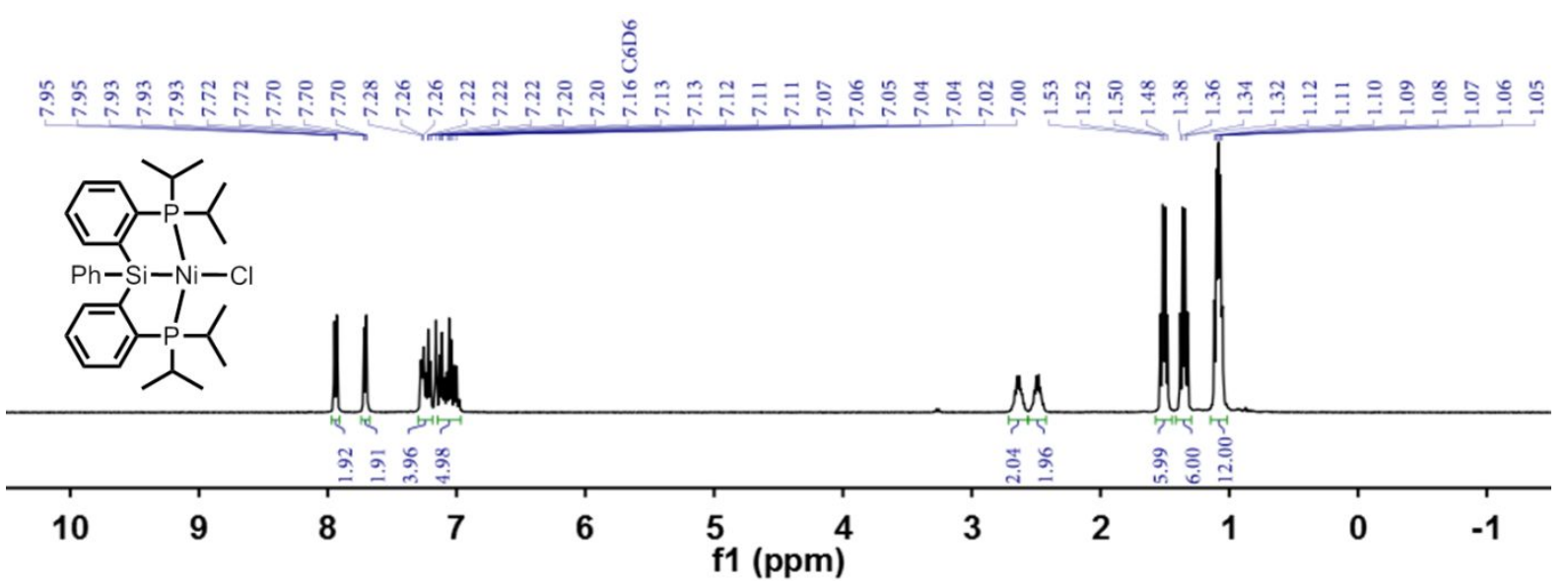

Figure S2. ${ }^{13} \mathrm{C}$ NMR spectrum $\left(\mathrm{C}_{6} \mathrm{D}_{6}, 101 \mathrm{MHz}\right)$ of $\left(\mathrm{PhSiP}_{2}\right) \mathrm{NiCl}(\mathbf{1})$ at room temperature.

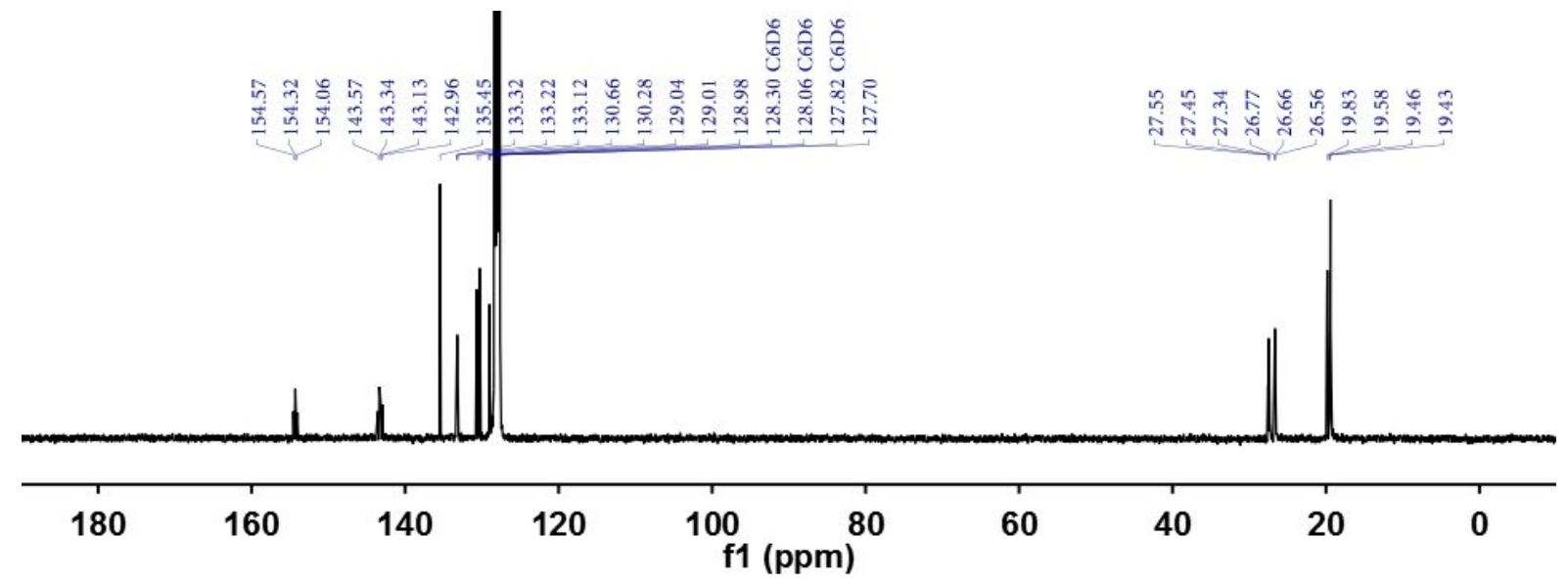

Figure S3. ${ }^{29} \mathrm{Si} \mathrm{NMR}$ spectrum $\left(\mathrm{C}_{6} \mathrm{D}_{6}, 79 \mathrm{MHz}\right)$ of $\left(\mathrm{PhSiP}_{2}\right) \mathrm{NiCl}(\mathbf{1})$ at room temperature.

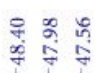

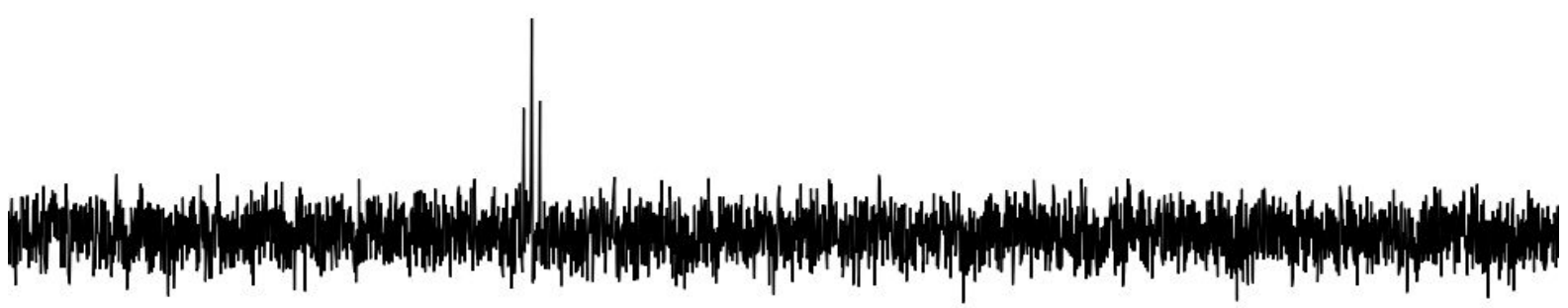

\begin{tabular}{|c|c|c|c|c|c|c|c|}
\hline 70 & 60 & 50 & 40 & 30 & 20 & 10 & 0 \\
\hline
\end{tabular}


Figure S4. ${ }^{31} \mathrm{P} \mathrm{NMR}$ spectrum $\left(\mathrm{C}_{6} \mathrm{D}_{6}, 162 \mathrm{MHz}\right)$ of $\left(\mathrm{PhSiP}_{2}\right) \mathrm{NiCl}(\mathbf{1})$ at room temperature.

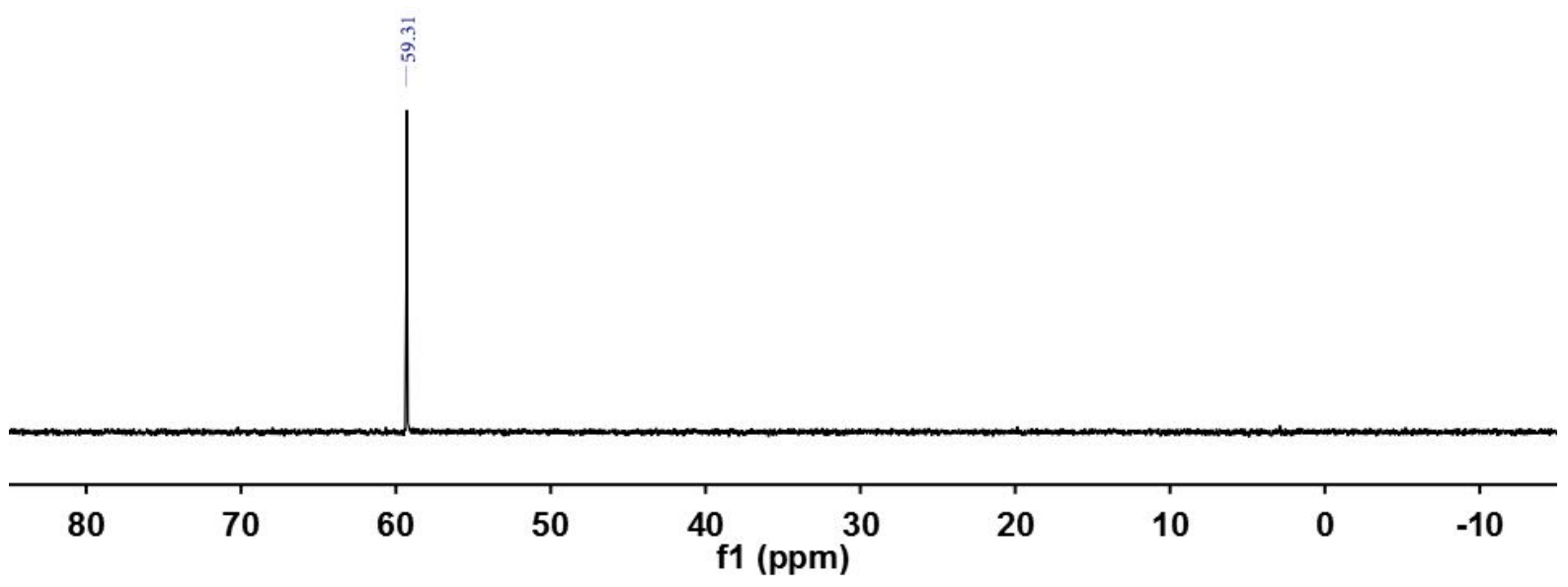

Figure S5. ${ }^{1} \mathrm{H}$ NMR spectrum $\left(\mathrm{C}_{6} \mathrm{D}_{6}, 400 \mathrm{MHz}\right)$ of $\left(\mathrm{PhSiP}_{2}\right) \mathrm{Ni}(\mathrm{NHMes})(\mathbf{2 a})$ at room temperature.

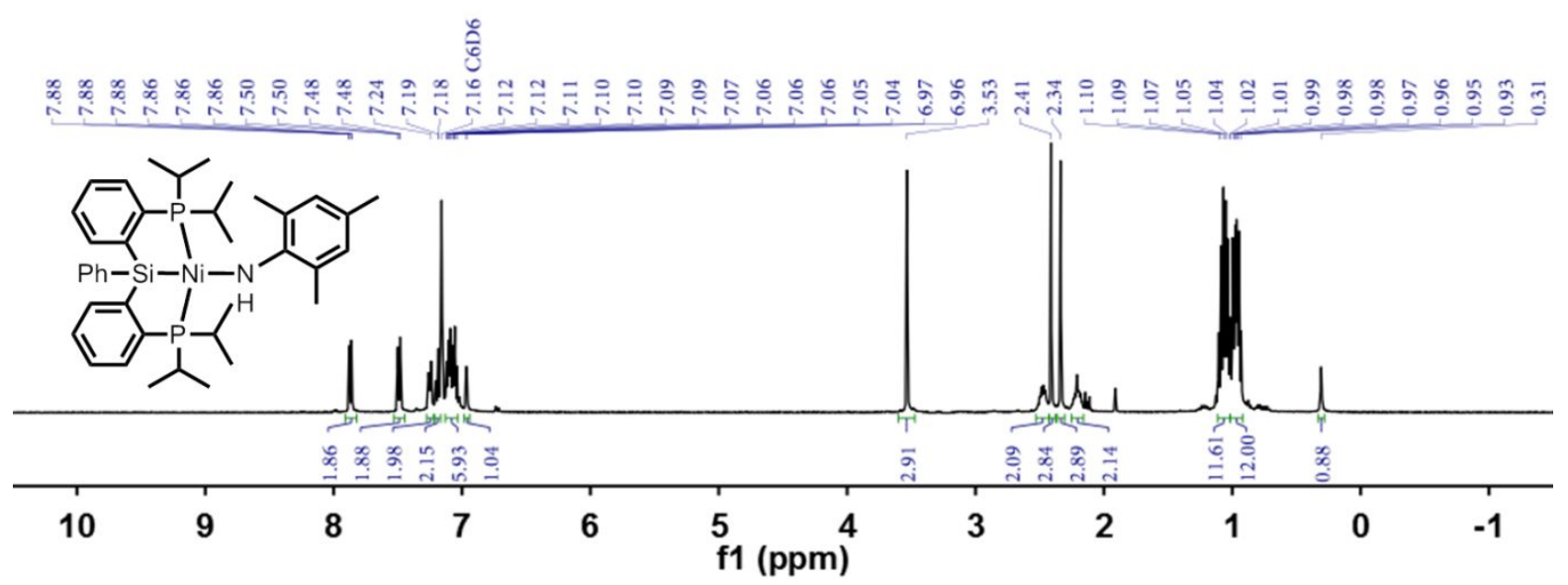

Figure S6. ${ }^{13} \mathrm{C}$ NMR spectrum $\left(\mathrm{C}_{6} \mathrm{D}_{6}, 101 \mathrm{MHz}\right)$ of $\left(\mathrm{PhSiP}_{2}\right) \mathrm{Ni}(\mathrm{NHMes})(\mathbf{2 a})$ at room temperature.

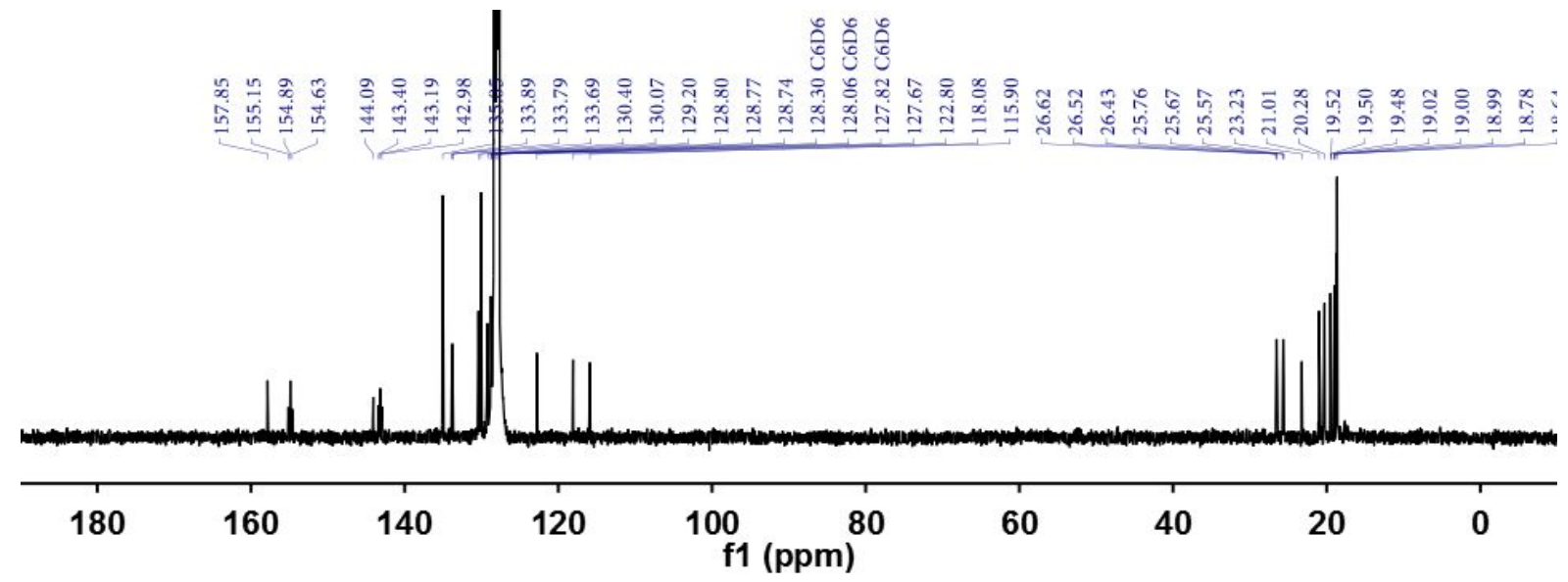


Figure S7. ${ }^{29} \mathrm{Si} \mathrm{NMR}$ spectrum $\left(\mathrm{C}_{6} \mathrm{D}_{6}, 79 \mathrm{MHz}\right)$ of $\left(\mathrm{PhSiP}_{2}\right) \mathrm{Ni}(\mathrm{NHMes})(\mathbf{2 a})$ at room temperature.

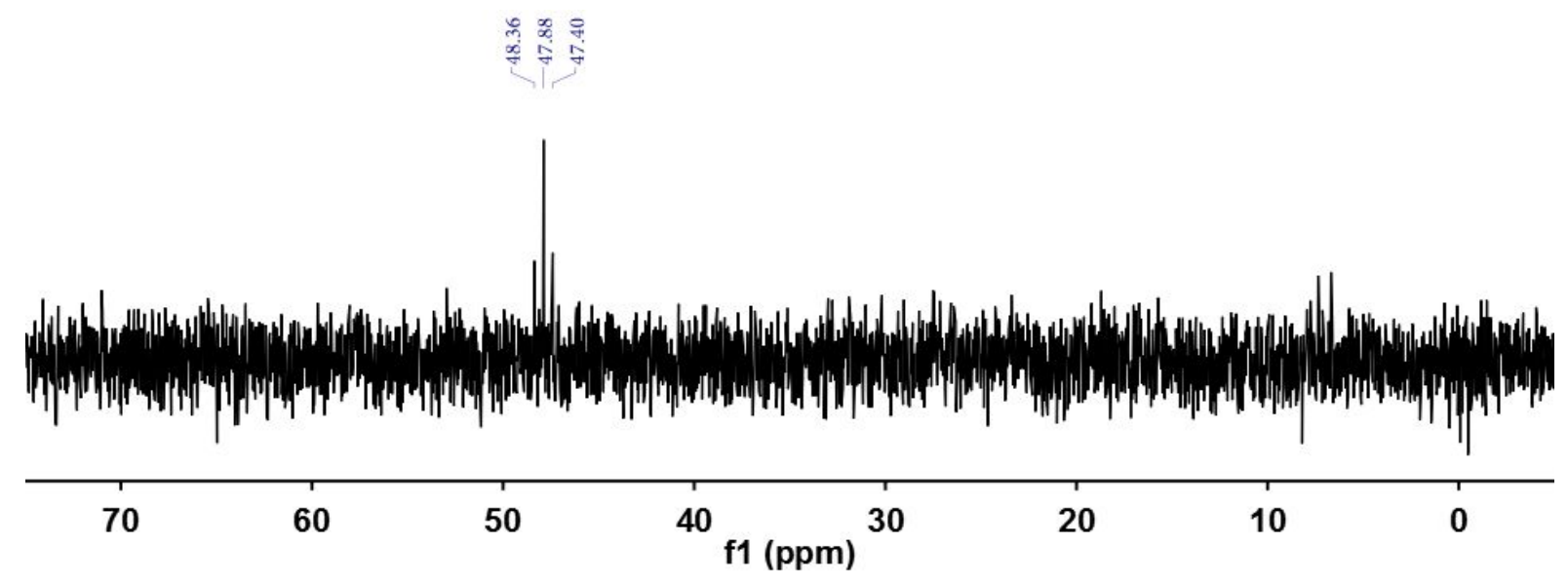

Figure S8. ${ }^{31} \mathrm{P}$ NMR spectrum $\left(\mathrm{C}_{6} \mathrm{D}_{6}, 162 \mathrm{MHz}\right)$ of $\left(\mathrm{PhSiP}_{2}\right) \mathrm{Ni}(\mathrm{NHMes})$ (2a) at room temperature.

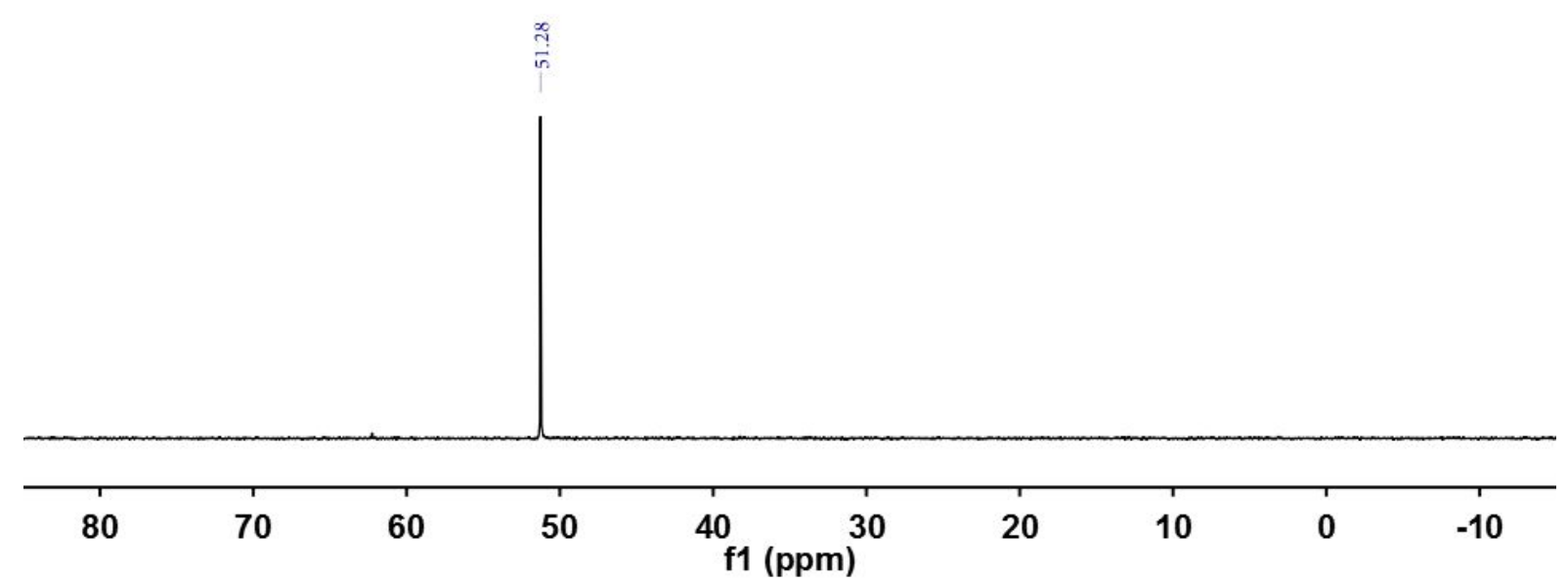

Figure S9. ${ }^{31} \mathrm{P}$ NMR spectrum $\left(\mathrm{C}_{6} \mathrm{D}_{6}, 162 \mathrm{MHz}\right)$ of in situ reaction mixture of 1, 2,4,6-triisopropylaniline and NaHMDS at room temperature. The spectrum was obtained within 10 minutes after the addition of NaHMDS.

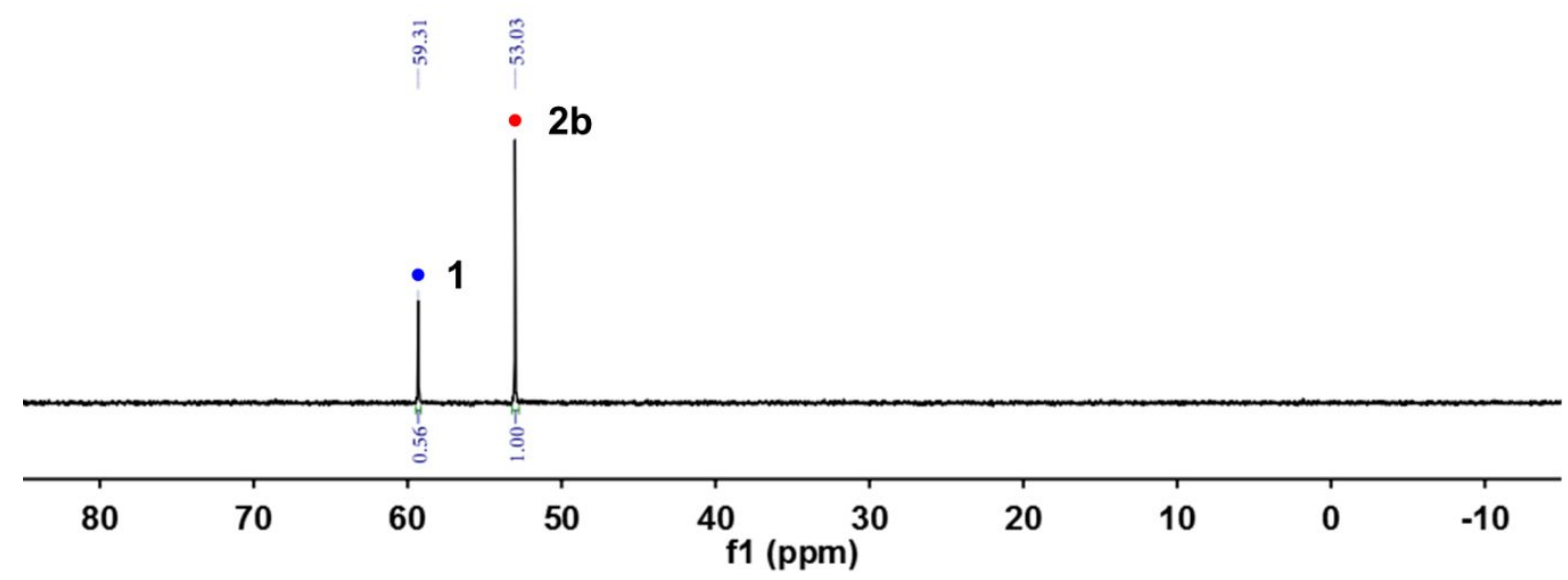


Figure S10. ${ }^{31} \mathrm{P}$ NMR spectra $\left(\mathrm{C}_{6} \mathrm{D}_{6}, 162 \mathrm{MHz}\right)$ of in situ reaction mixture of 1, 2,4,6-triisopropylaniline and NaHMDS at various temperature.

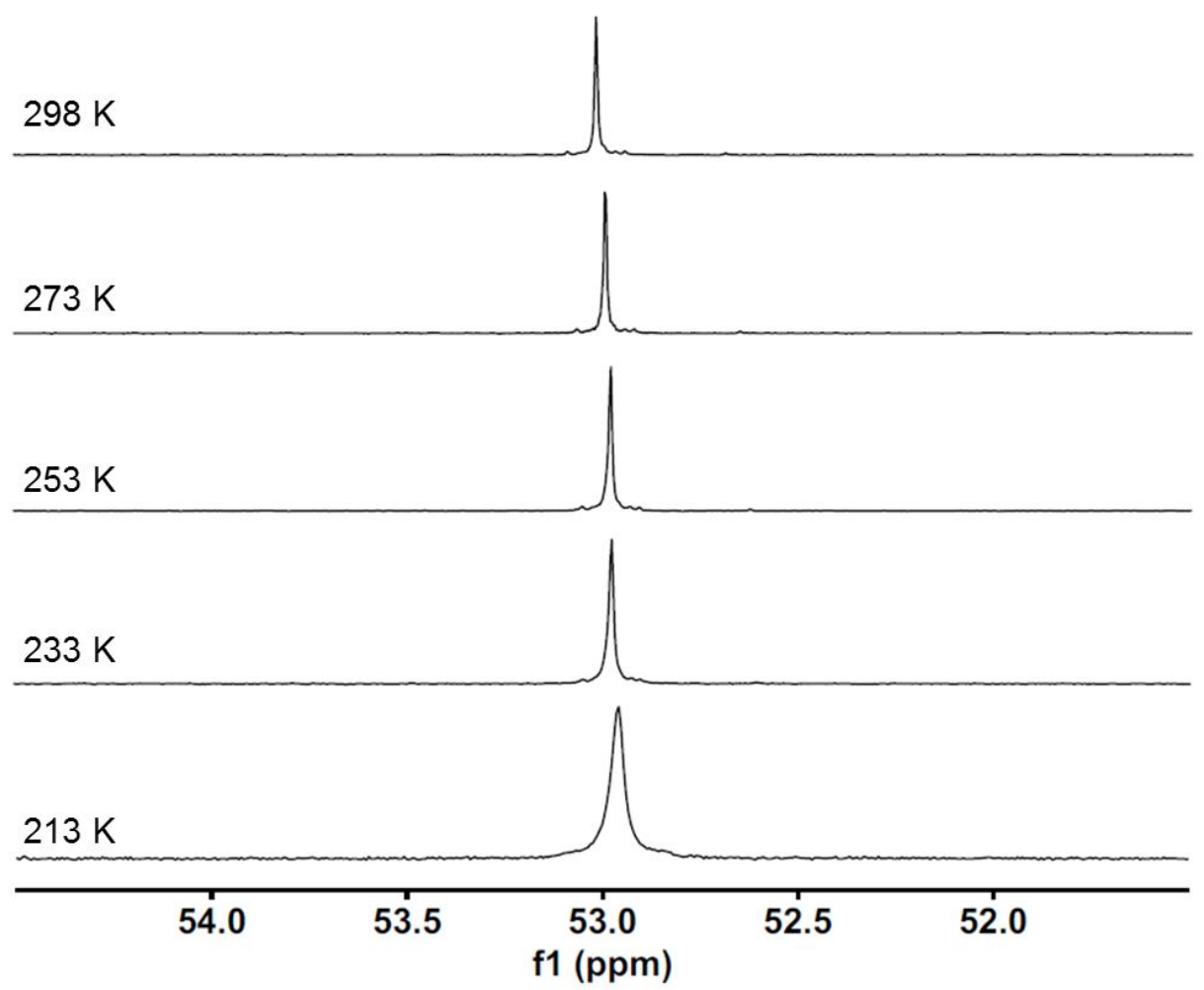

Figure S11. ${ }^{1} \mathrm{H}$ NMR spectrum $\left(\mathrm{C}_{6} \mathrm{D}_{6}, 400 \mathrm{MHz}\right)$ of $\left((\operatorname{TripNH}) \mathrm{SiP}_{2}\right) \mathrm{Ni}(\mathrm{Ph})(\mathbf{3 b})$ at room temperature.

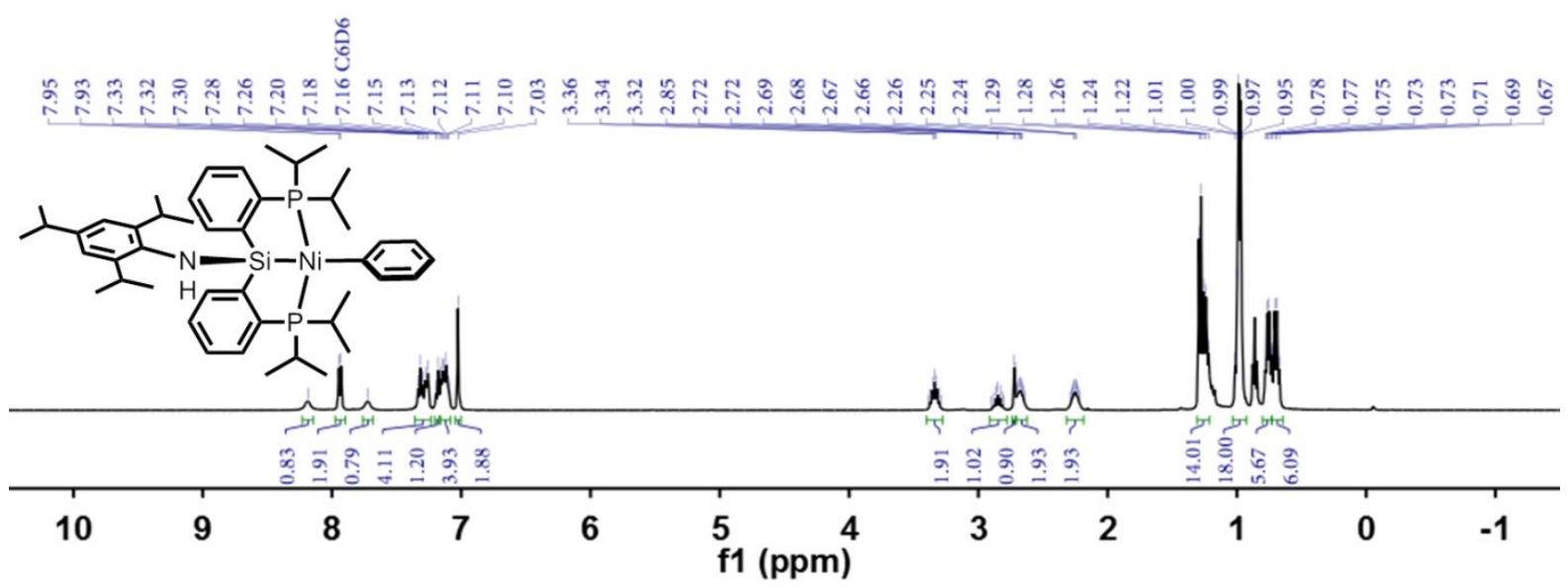


Figure S12. ${ }^{13} \mathrm{C}$ NMR spectrum $\left(\mathrm{C}_{6} \mathrm{D}_{6}, 101 \mathrm{MHz}\right)$ of $\left((\right.$ TripNH$\left.) \mathrm{SiP}_{2}\right) \mathrm{Ni}(\mathrm{Ph})(\mathbf{3 b})$ at room temperature.

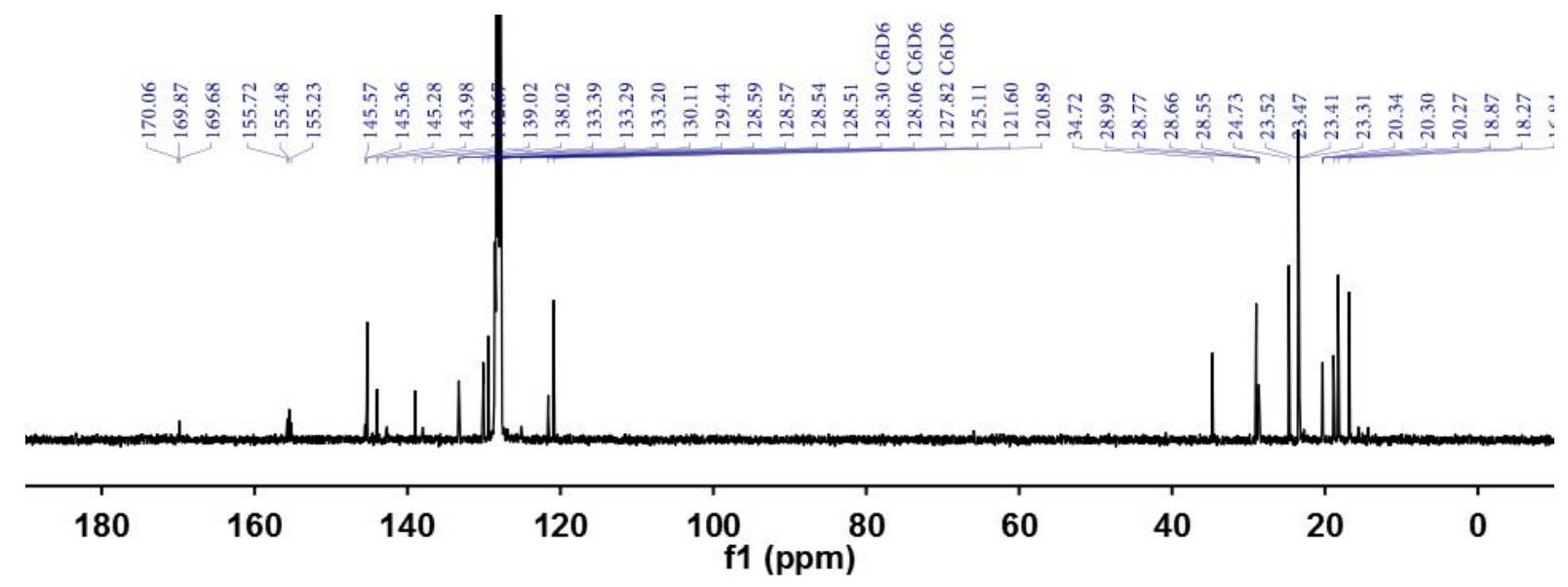

Figure S13. ${ }^{29} \mathrm{Si} \mathrm{NMR}$ spectrum $\left(\mathrm{C}_{6} \mathrm{D}_{6}, 79 \mathrm{MHz}\right)$ of $\left((\mathrm{TripNH}) \mathrm{SiP}_{2}\right) \mathrm{Ni}(\mathrm{Ph})(\mathbf{3 b})$ at room temperature.

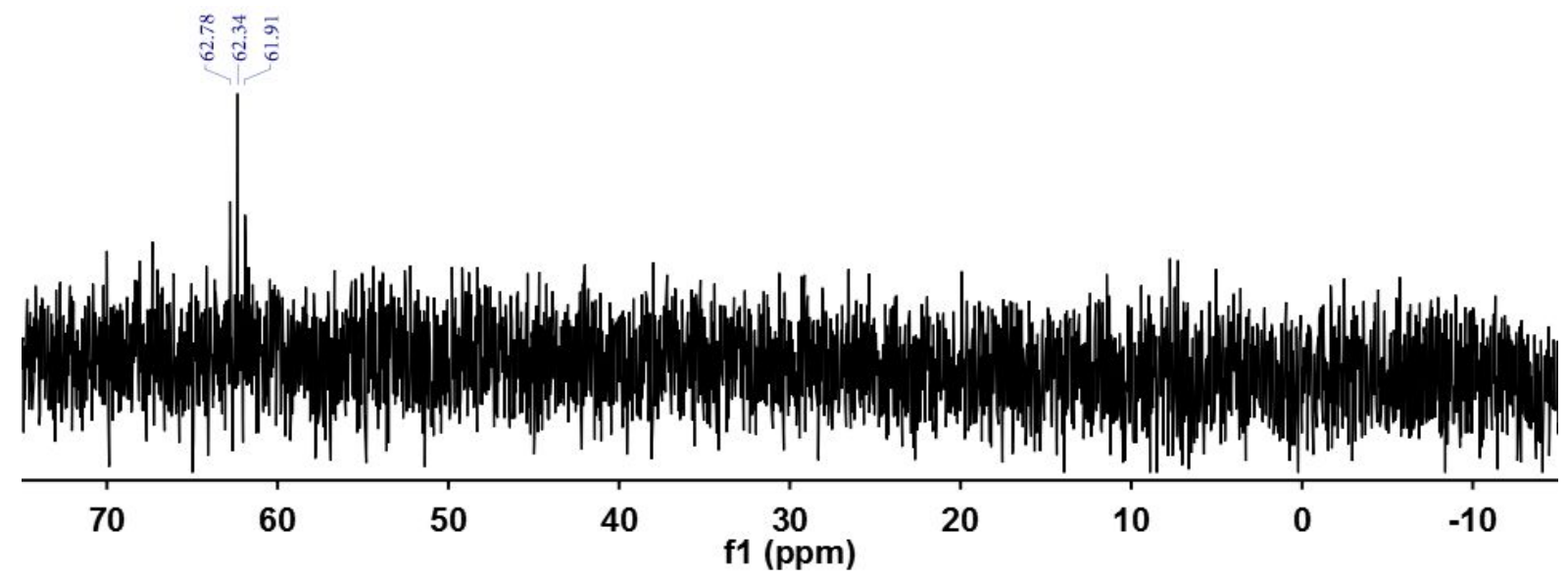

Figure S14. ${ }^{31} \mathrm{P}$ NMR spectrum $\left(\mathrm{C}_{6} \mathrm{D}_{6}, 162 \mathrm{MHz}\right)$ of $\left(\left(\right.\right.$ TripNH) $\left.\mathrm{SiP}_{2}\right) \mathrm{Ni}(\mathrm{Ph})(\mathbf{3 b})$ at room temperature.

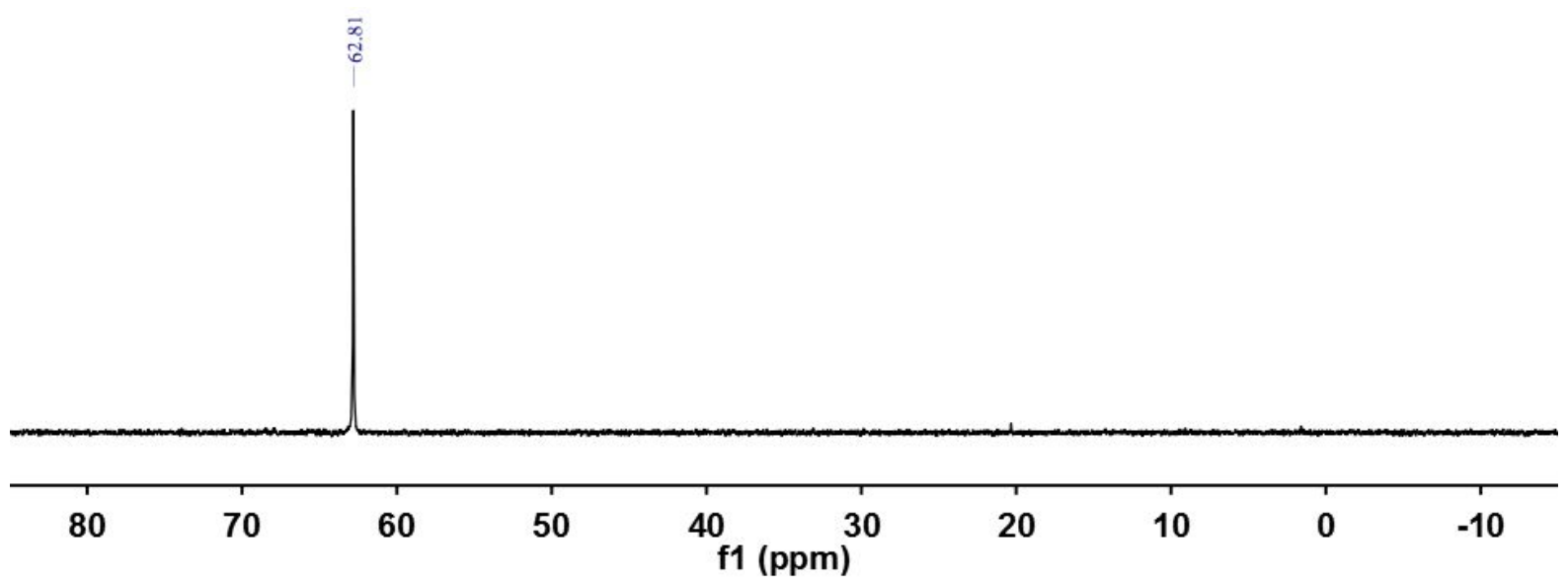


Figure S15. ${ }^{1} \mathrm{H}$ NMR spectrum $\left(\mathrm{C}_{6} \mathrm{D}_{6}, 400 \mathrm{MHz}\right)$ of $\left((\mathrm{MesNH}) \mathrm{SiP}_{2}\right) \mathrm{Ni}(\mathrm{Ph})(\mathbf{3 a})$ at room temperature.

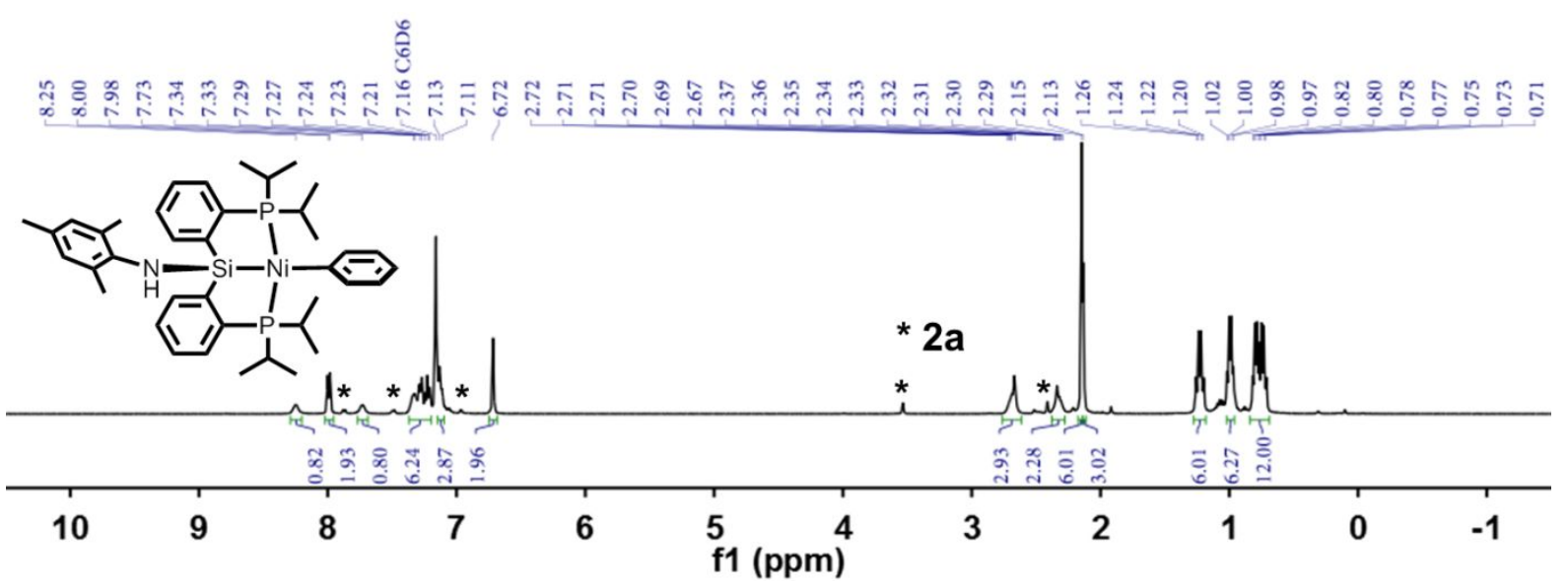

Figure S16. ${ }^{29} \mathrm{Si} \mathrm{NMR}$ spectrum $\left(\mathrm{C}_{6} \mathrm{D}_{6}, 79 \mathrm{MHz}\right)$ of $\left((\mathrm{MesNH}) \mathrm{SiP}_{2}\right) \mathrm{Ni}(\mathrm{Ph})(\mathbf{3 a})$ at room temperature.

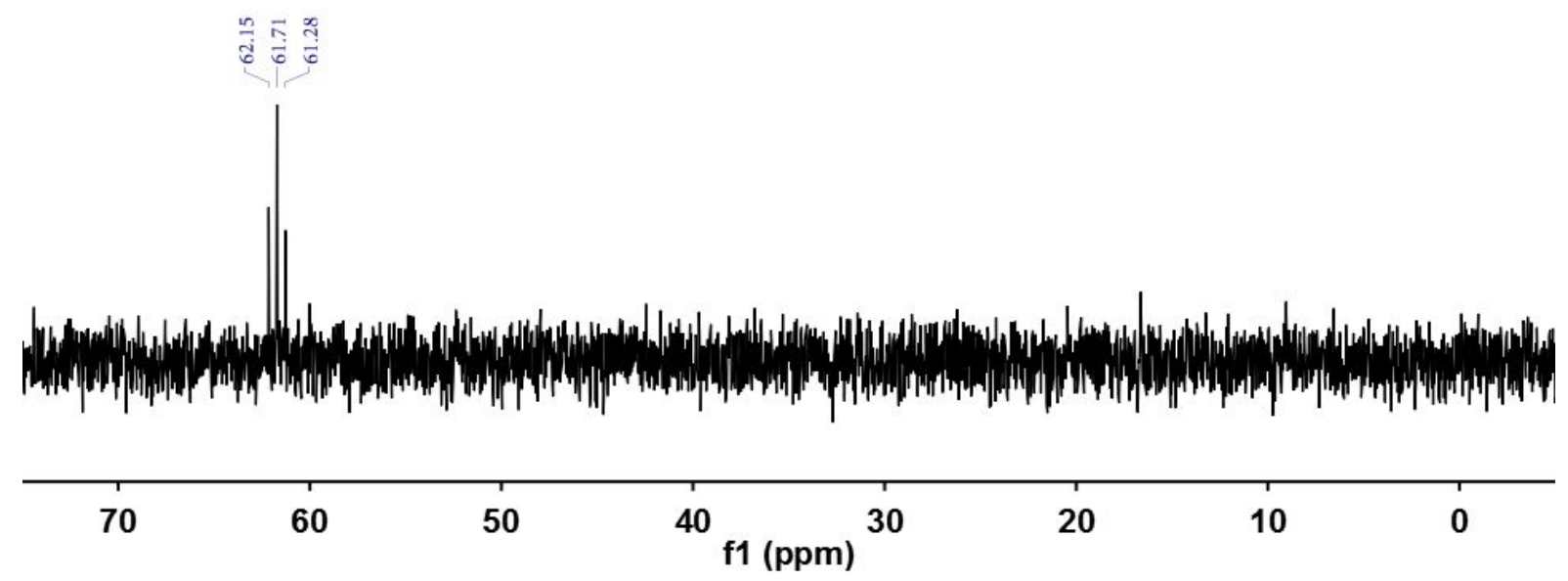

Figure S17. ${ }^{31} \mathrm{P}$ NMR spectrum $\left(\mathrm{C}_{6} \mathrm{D}_{6}, 162 \mathrm{MHz}\right)$ of $\left((\mathrm{MesNH}) \mathrm{SiP}_{2}\right) \mathrm{Ni}(\mathrm{Ph})(3 \mathrm{a})$ at room temperature.

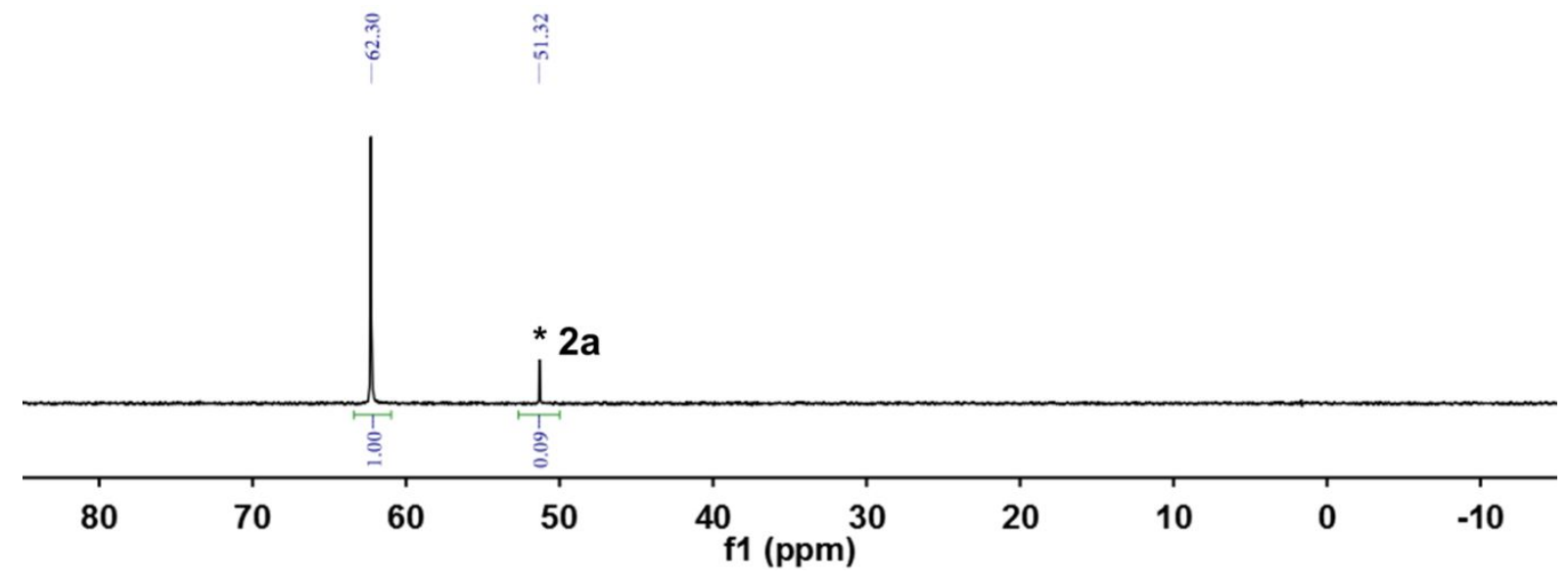


Figure S18. ${ }^{31} \mathrm{P}$ NMR spectrum $\left(\mathrm{C}_{6} \mathrm{D}_{6}, 162 \mathrm{MHz}\right)$ of in situ reaction mixture of $\mathbf{2 a}$ and $\mathrm{CO}(\mathrm{g})$ at room temperature.

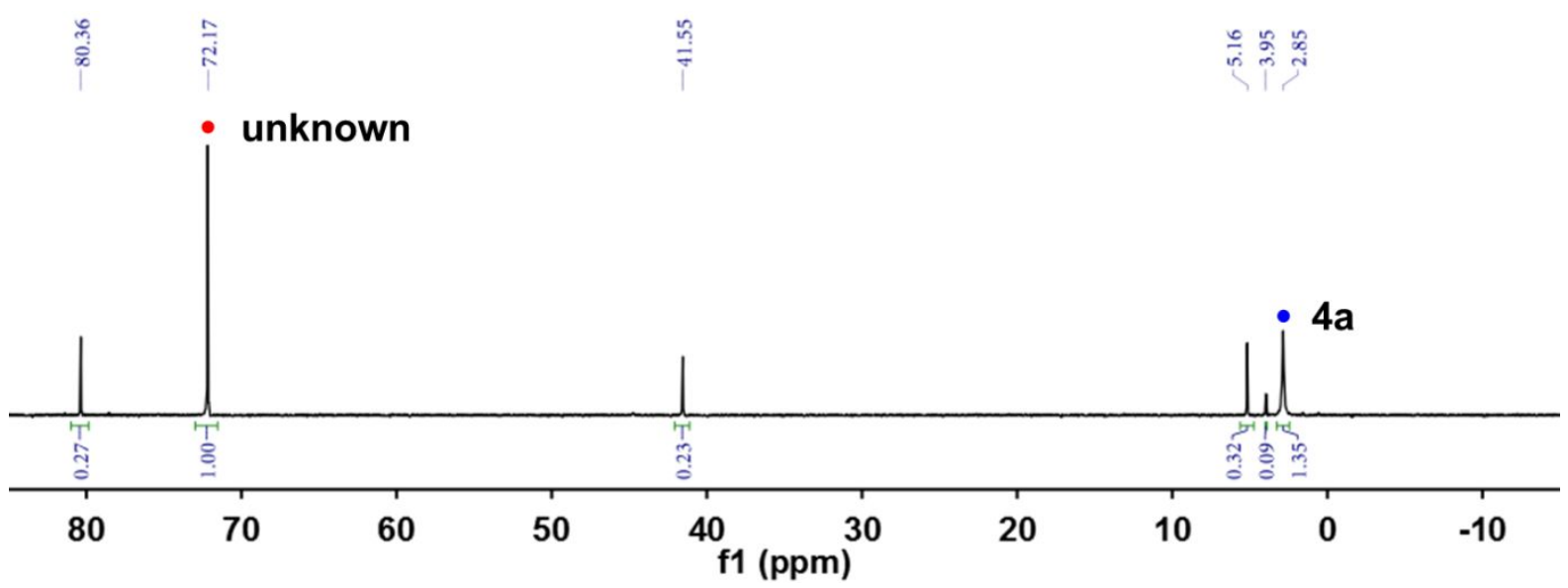

Figure S19. ${ }^{31} \mathrm{P}$ NMR spectrum $\left(\mathrm{C}_{6} \mathrm{D}_{6}, 162 \mathrm{MHz}\right)$ of a unknown species at room temperature, obtained from in situ reaction mixture of 1, 2,4,6-triisopropylaniline and NaHMDS in $\mathrm{CO}$ atmosphere.

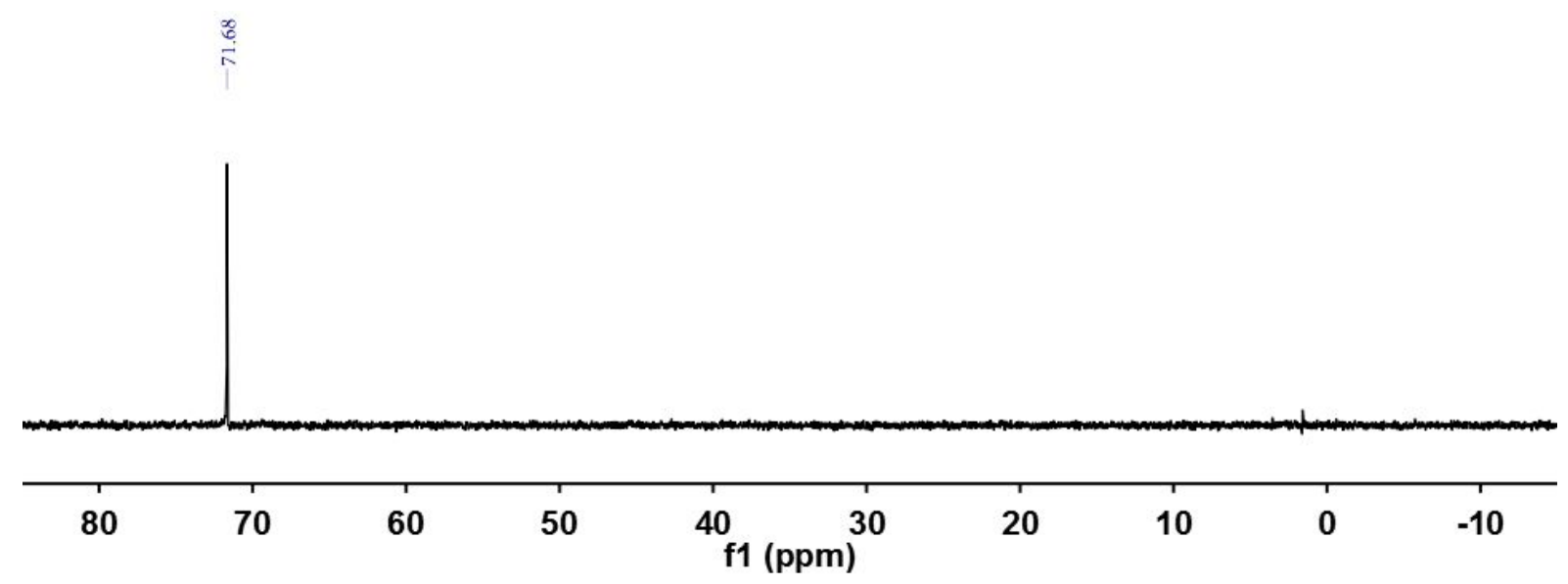

Figure S20. ${ }^{1} \mathrm{H}$ NMR spectrum $\left(\mathrm{C}_{6} \mathrm{D}_{6}, 400 \mathrm{MHz}\right)$ of $(\mathrm{TripNH}) \mathrm{PhSiP}_{2}(4 \mathbf{b})$ at room temperature.

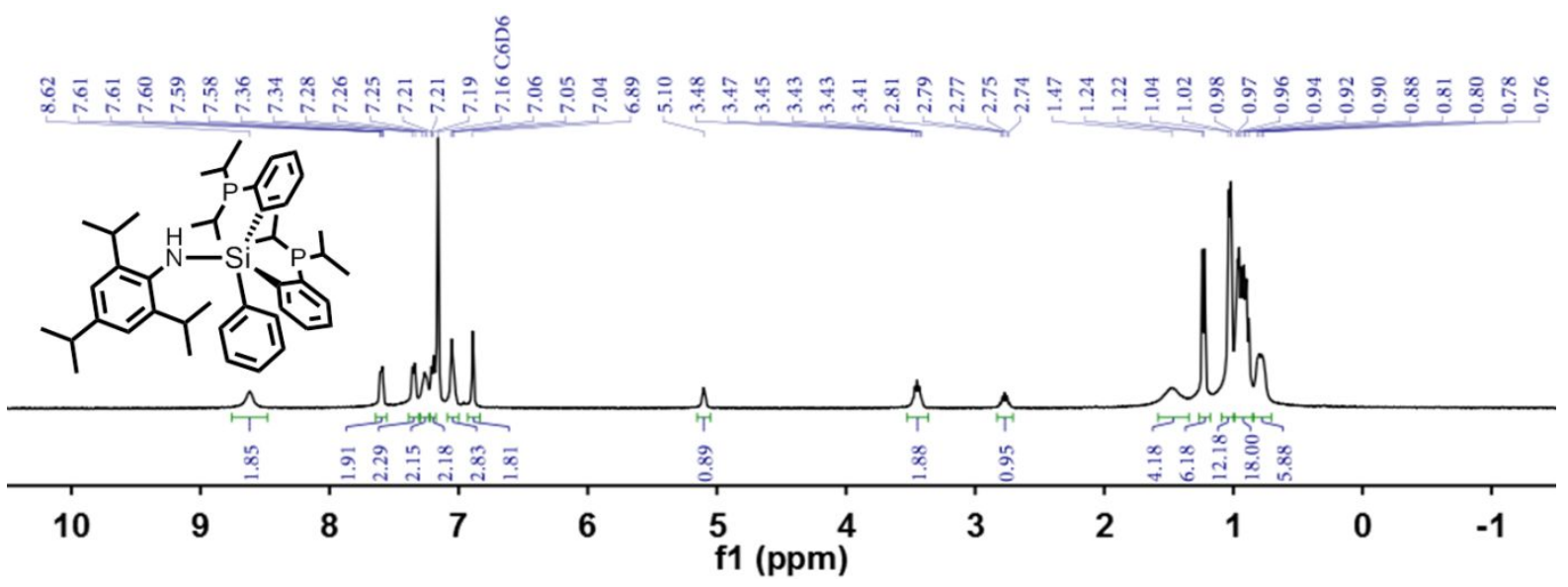


Figure S21. ${ }^{13} \mathrm{C}$ NMR spectrum $\left(\mathrm{C}_{6} \mathrm{D}_{6}, 101 \mathrm{MHz}\right)$ of $(\mathrm{TripNH}) \mathrm{PhSiP}_{2}(\mathbf{4 b})$ at room temperature.

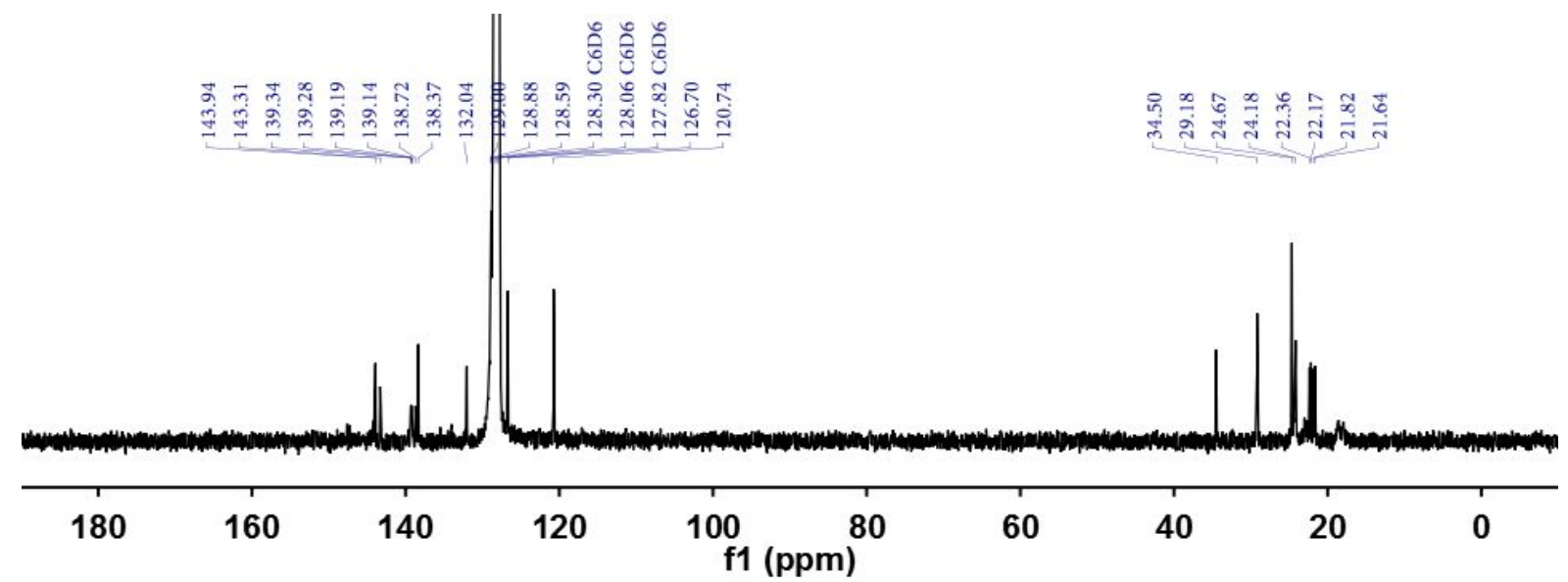

Figure S22. ${ }^{29} \mathrm{Si}$ NMR spectrum $\left(\mathrm{C}_{6} \mathrm{D}_{6}, 79 \mathrm{MHz}\right)$ of (TripNH) $\mathrm{PhSiP}_{2}(\mathbf{4 b})$ at room temperature.

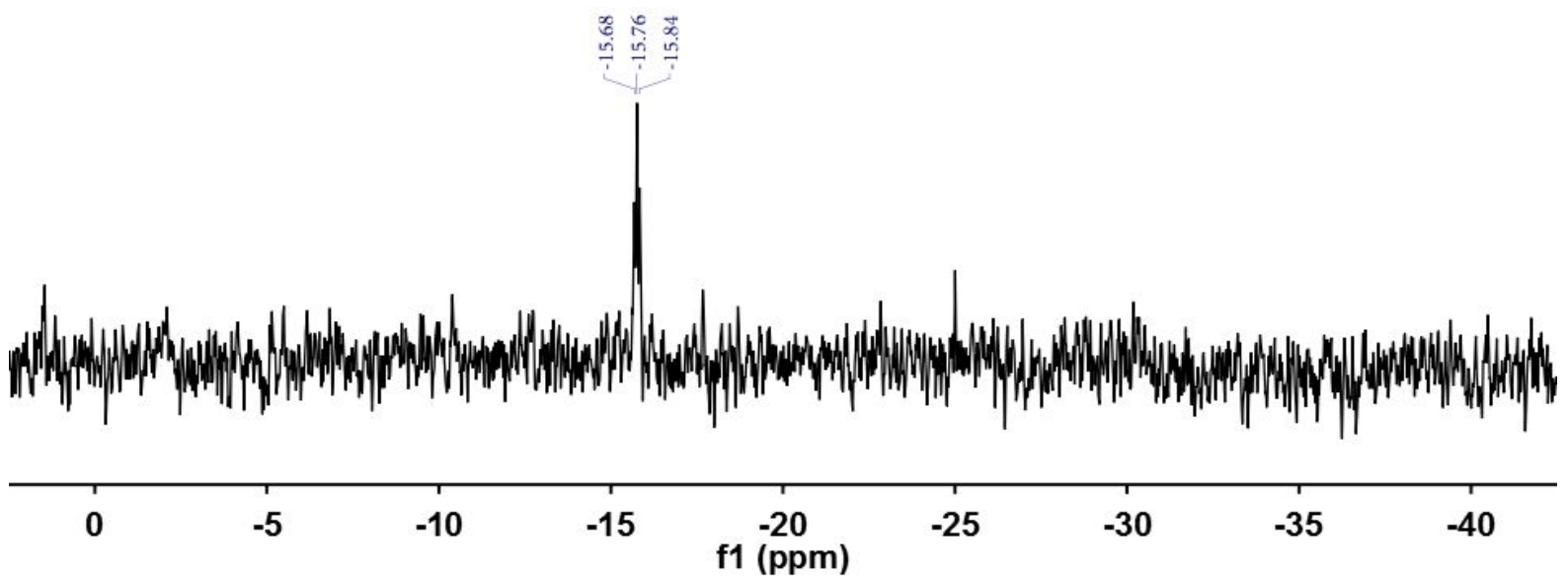

Figure S23. ${ }^{31} \mathrm{P}$ NMR spectrum $\left(\mathrm{C}_{6} \mathrm{D}_{6}, 162 \mathrm{MHz}\right)$ of (TripNH) $\mathrm{PhSiP}_{2}(\mathbf{4 b})$ at room temperature.

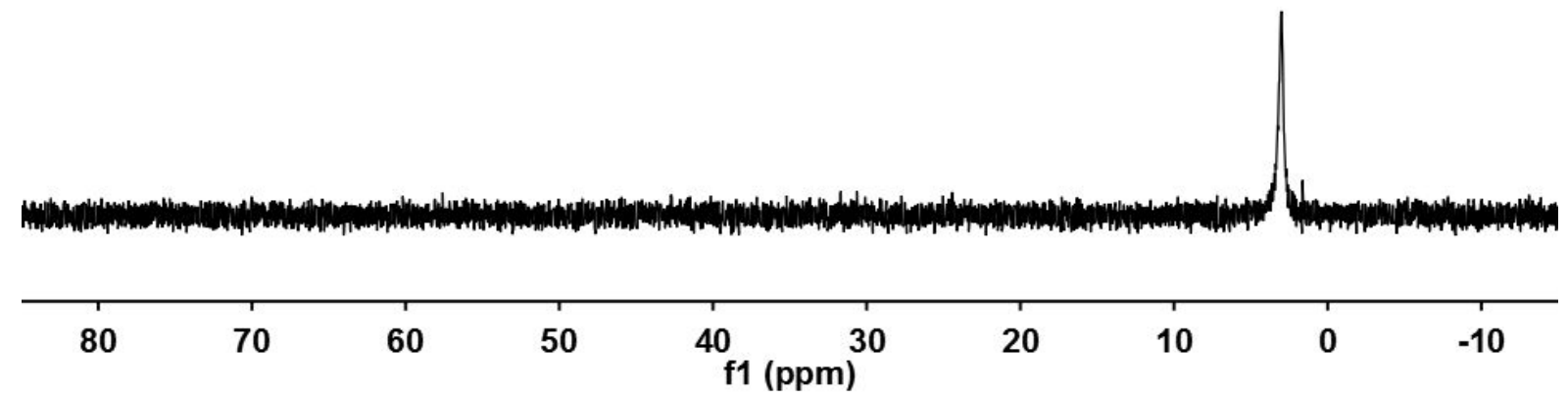


Figure S24. ${ }^{1} \mathrm{H}$ NMR spectrum $\left(\mathrm{C}_{6} \mathrm{D}_{6}, 400 \mathrm{MHz}\right)$ of $(\mathrm{MesNH}) \mathrm{PhSiP}_{2}(4 \mathbf{a})$ at room temperature.

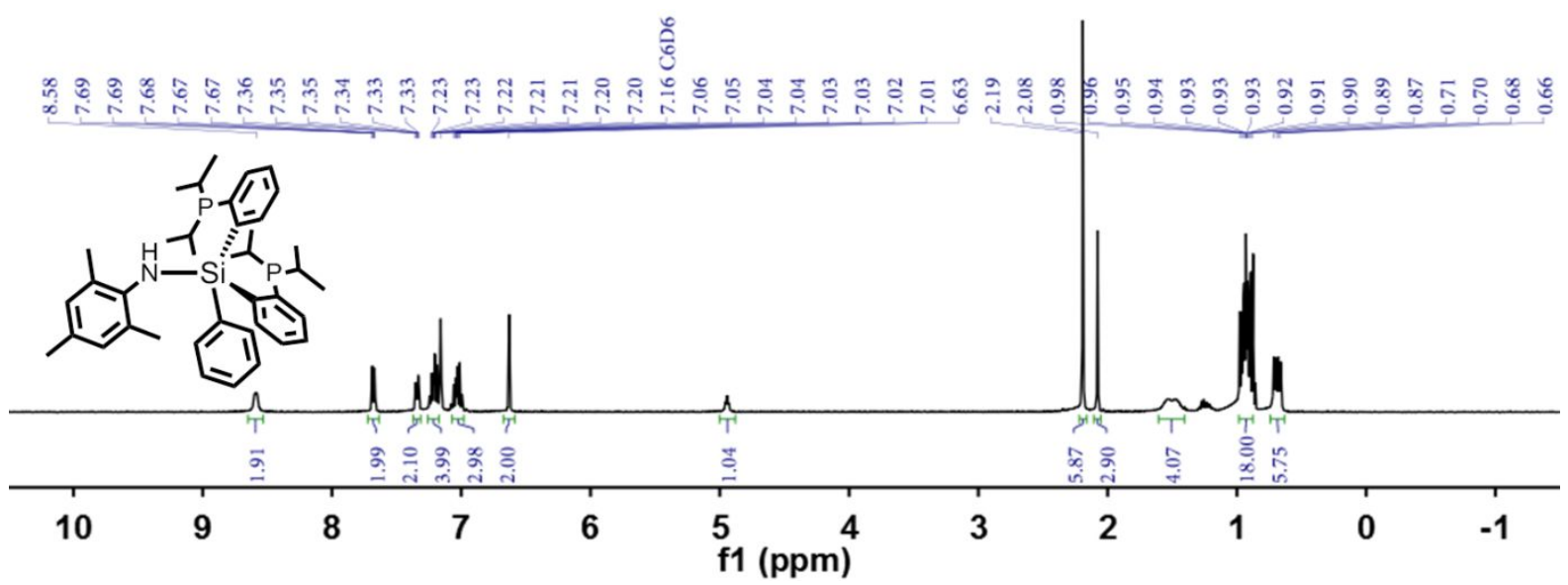

Figure S25. ${ }^{13} \mathrm{C}$ NMR spectrum $\left(\mathrm{C}_{6} \mathrm{D}_{6}, 101 \mathrm{MHz}\right)$ of $(\mathrm{MesNH}) \mathrm{PhSiP}_{2}(\mathbf{4 a})$ at room temperature.

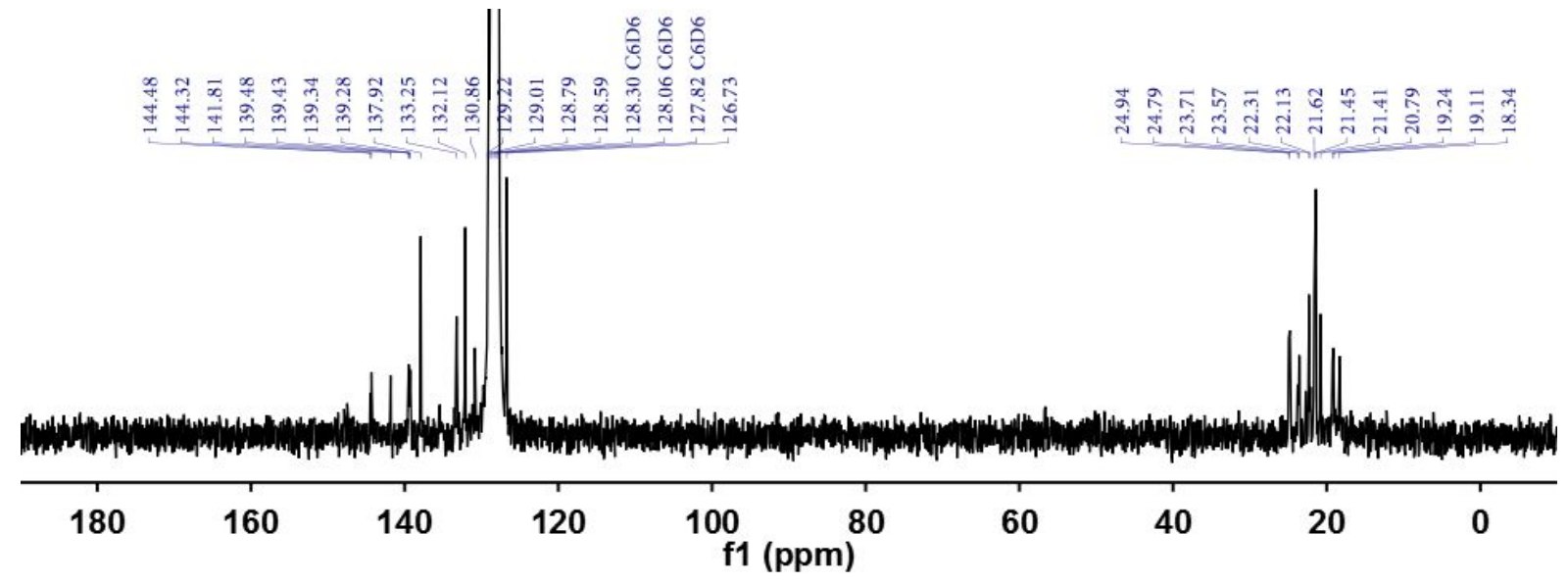

Figure S26. ${ }^{29} \mathrm{Si} \mathrm{NMR}$ spectrum $\left(\mathrm{C}_{6} \mathrm{D}_{6}, 79 \mathrm{MHz}\right)$ of $(\mathrm{MesNH}) \mathrm{PhSiP}_{2}($ 4a) at room temperature.

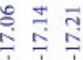

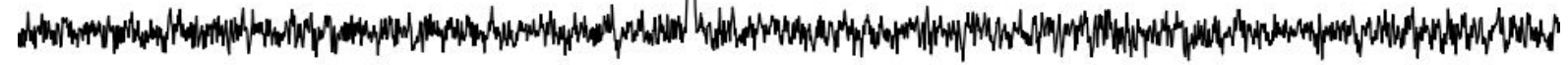

\begin{tabular}{|c|c|c|c|c|c|c|c|c|}
\hline 0 & -5 & -10 & -15 & $\begin{array}{l}-20 \\
\text { f1 (ppm) }\end{array}$ & -25 & -30 & -35 & -40 \\
\hline
\end{tabular}


Figure S27. ${ }^{31} \mathrm{P}$ NMR spectrum $\left(\mathrm{C}_{6} \mathrm{D}_{6}, 162 \mathrm{MHz}\right)$ of $(\mathrm{MesNH}) \mathrm{PhSiP}_{2}(\mathbf{4 a})$ at room temperature.

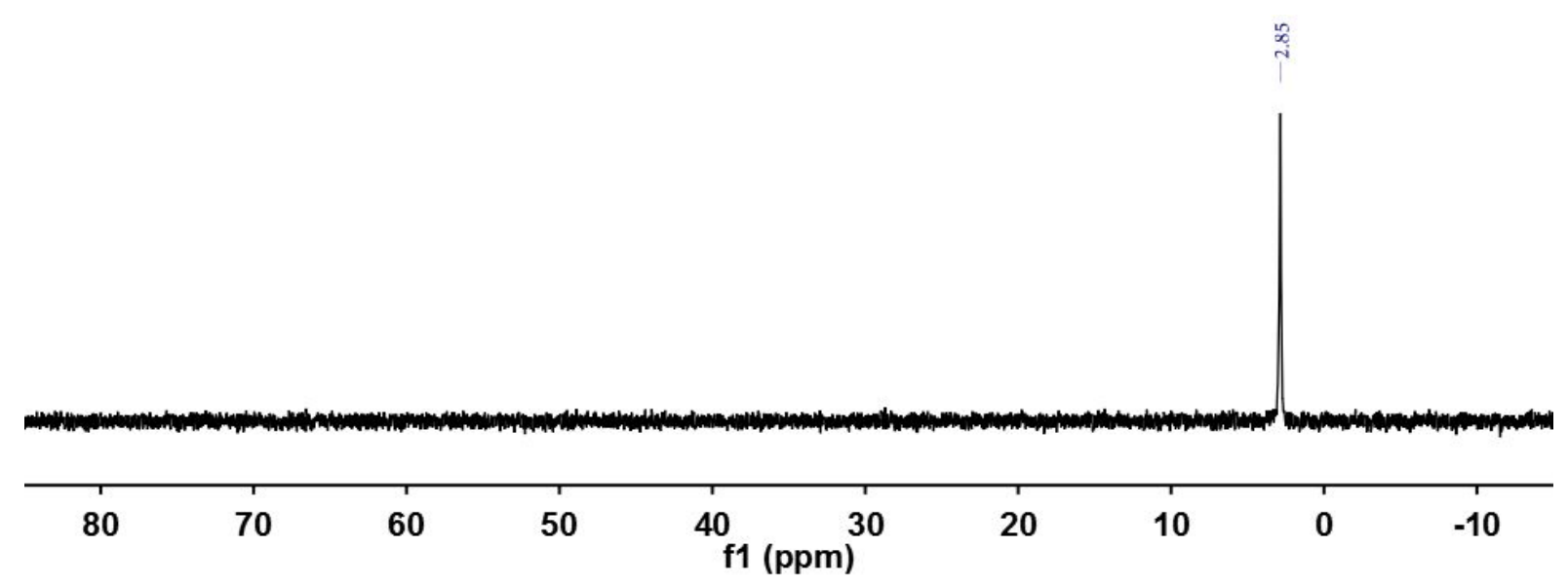

Figure S28. ${ }^{31} \mathrm{P}$ NMR spectrum $\left(\mathrm{C}_{6} \mathrm{D}_{6}, 162 \mathrm{MHz}\right)$ of in situ reaction mixture of $\mathbf{3 a}$ and tert-butyl isocyanide at room temperature.

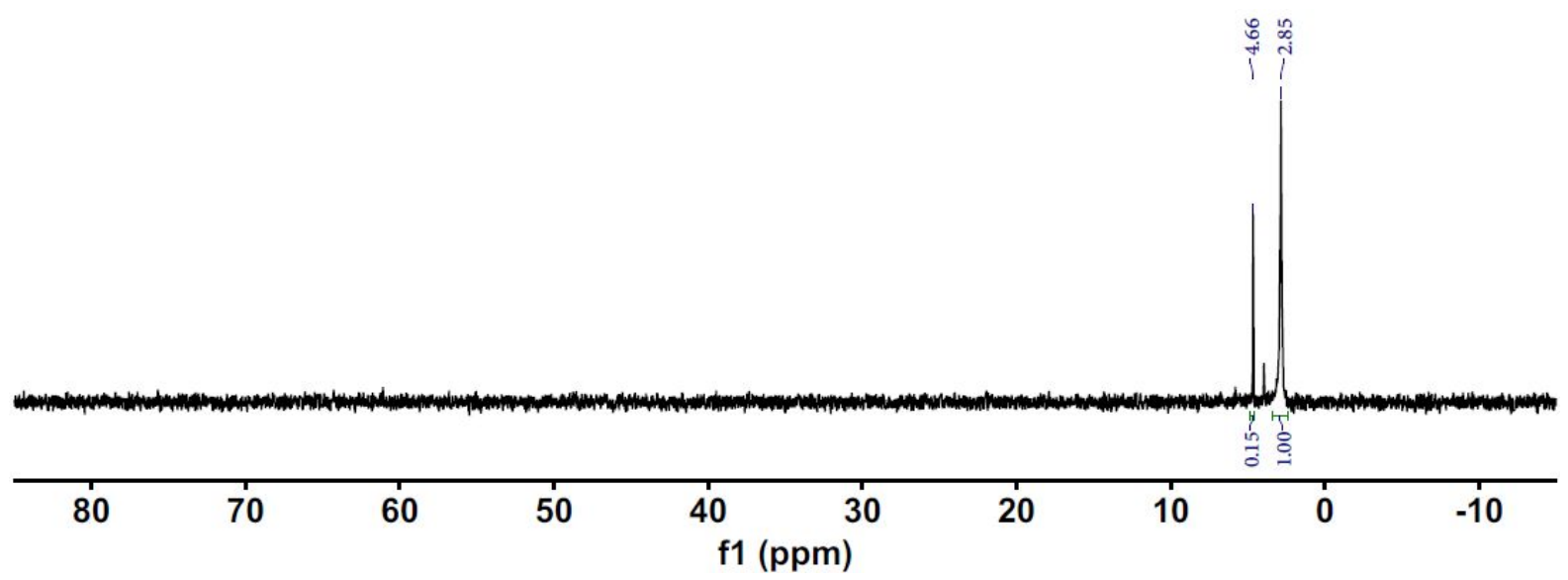

Figure S29. ${ }^{31} \mathrm{P}$ NMR spectrum $\left(\mathrm{C}_{6} \mathrm{D}_{6}, 162 \mathrm{MHz}\right)$ of in situ reaction mixture of $\mathbf{3 b}$ and tert-butyl isocyanide at room temperature.

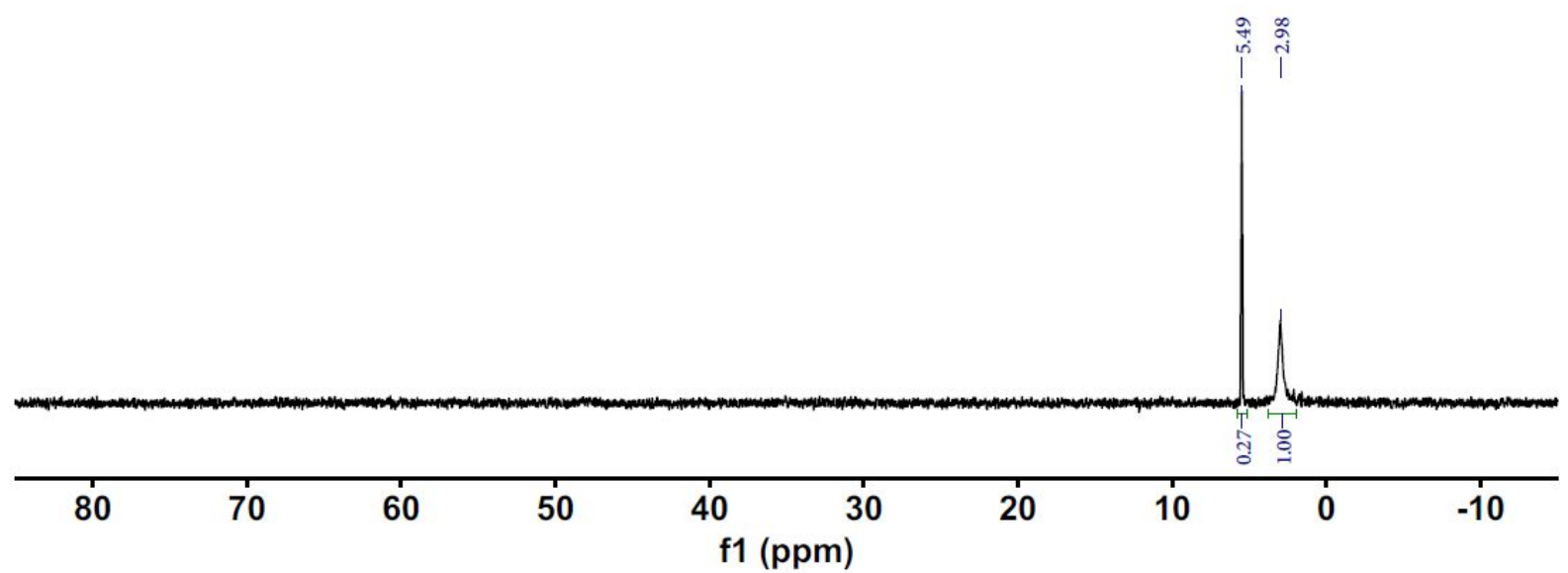


Figure S30. ${ }^{1} \mathrm{H}$ NMR spectrum $\left(\mathrm{C}_{6} \mathrm{D}_{6}, 400 \mathrm{MHz}\right)$ of $\left((\mathrm{TripNH})\left(\eta^{2}-\mathrm{Ph}\right) \mathrm{SiP}_{2}\right) \mathrm{Ni}\left(\mathrm{CN}^{t} \mathrm{Bu}\right)(5)$ at room temperature.

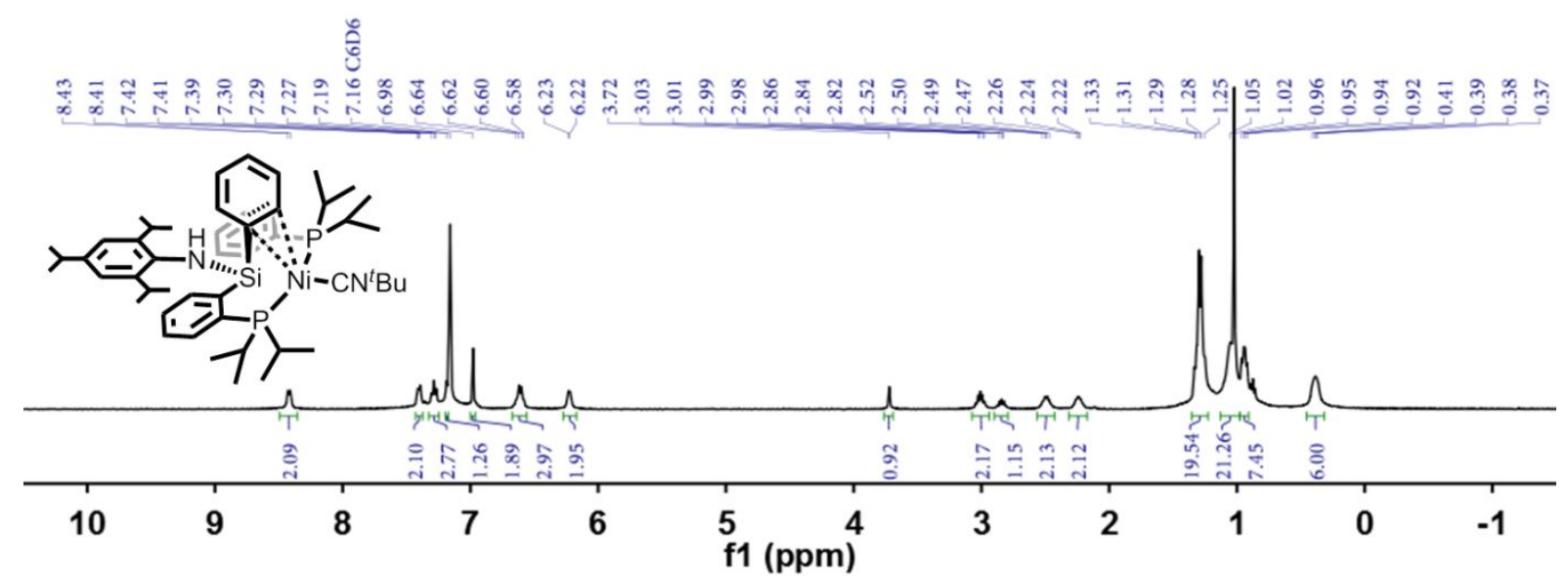

Figure S31. ${ }^{13} \mathrm{C}$ NMR spectrum $\left(\mathrm{C}_{6} \mathrm{D}_{6}, 101 \mathrm{MHz}\right)$ of $\left((\mathrm{TripNH})\left(\eta^{2}-\mathrm{Ph}\right) \mathrm{SiP}_{2}\right) \mathrm{Ni}\left(\mathrm{CN}^{t} \mathrm{Bu}\right)(\mathbf{5})$ at room temperature. (*: Ar-C of $\eta^{2}-\mathrm{Ph}^{1}, *: C\left(\mathrm{CH}_{3}\right)_{3}$ of tert-butyl isocyanide ${ }^{2}$ )

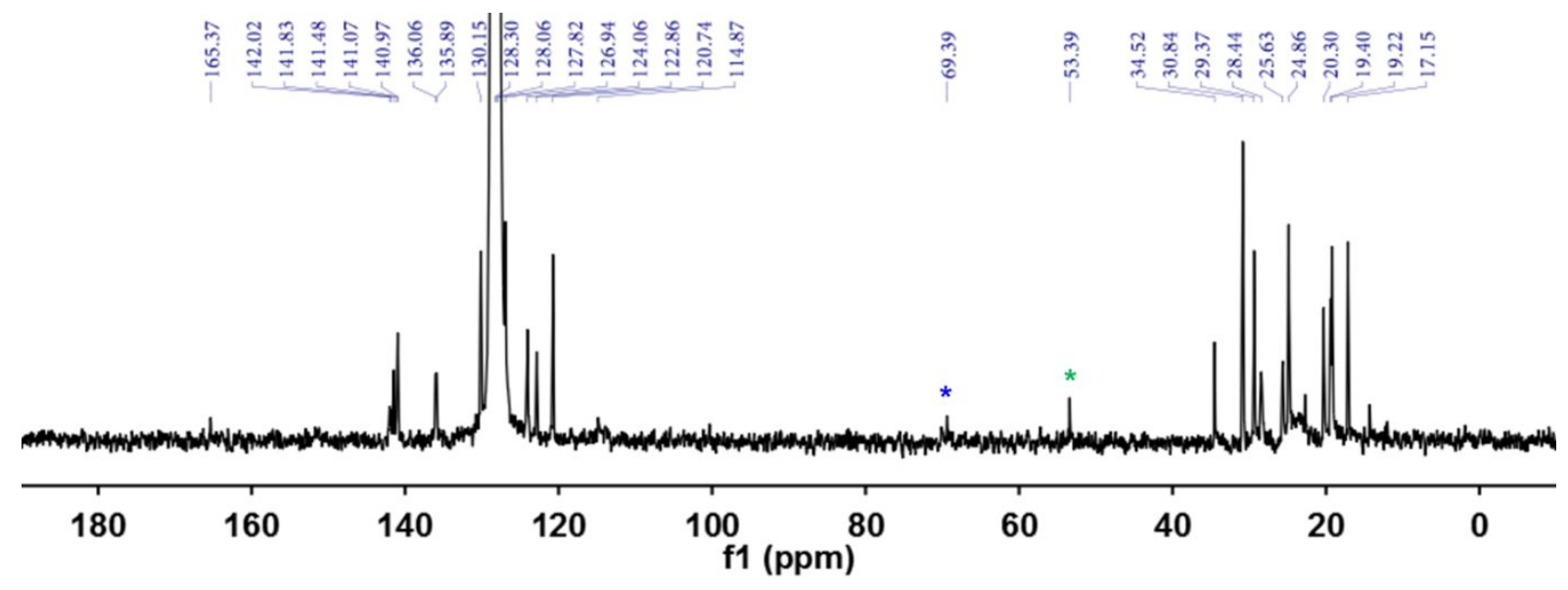

Figure S32. ${ }^{29} \mathrm{Si} \mathrm{NMR}$ spectrum $\left(\mathrm{C}_{6} \mathrm{D}_{6}, 79 \mathrm{MHz}\right)$ of $\left((\mathrm{TripNH})\left(\eta^{2}-\mathrm{Ph}\right) \mathrm{SiP}_{2}\right) \mathrm{Ni}\left(\mathrm{CN}^{t} \mathrm{Bu}\right)(5)$ at room temperature.

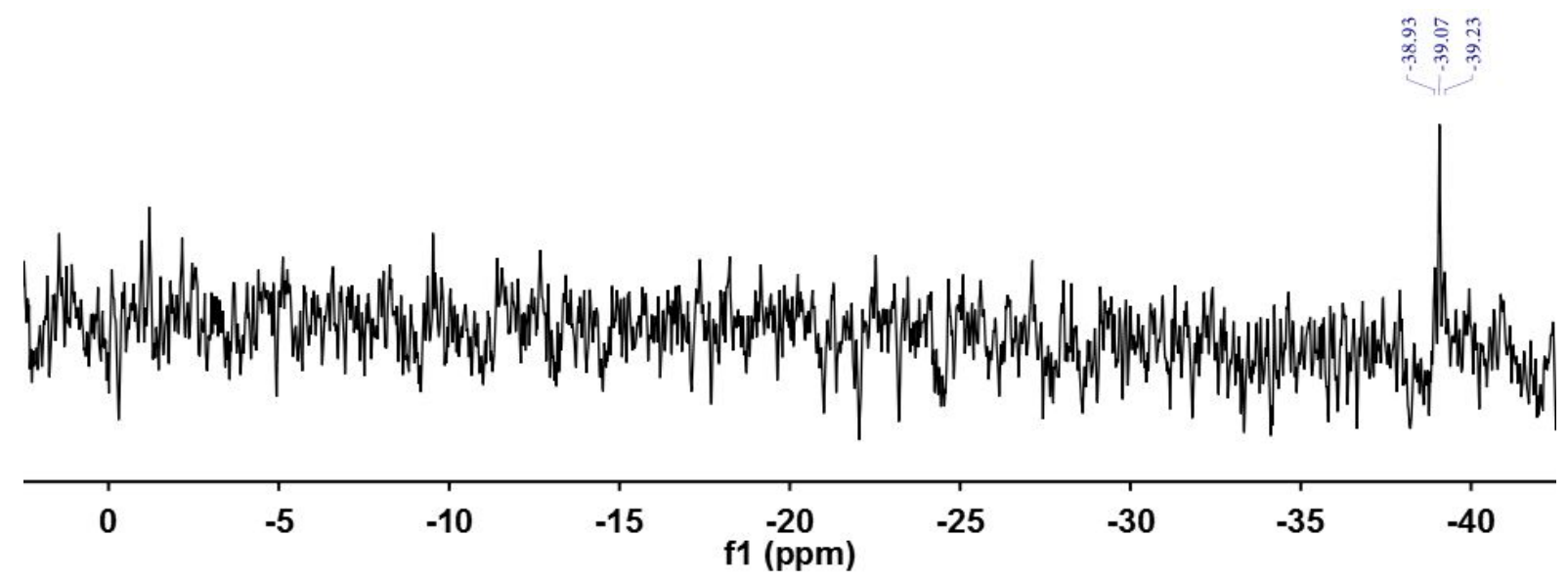


Figure S33. ${ }^{31} \mathrm{P}$ NMR spectrum $\left(\mathrm{C}_{6} \mathrm{D}_{6}, 162 \mathrm{MHz}\right)$ of $\left((\mathrm{TripNH})\left(\eta^{2}-\mathrm{Ph}\right) \mathrm{SiP}_{2}\right) \mathrm{Ni}\left(\mathrm{CN}^{t} \mathrm{Bu}\right)(5)$ at room temperature.

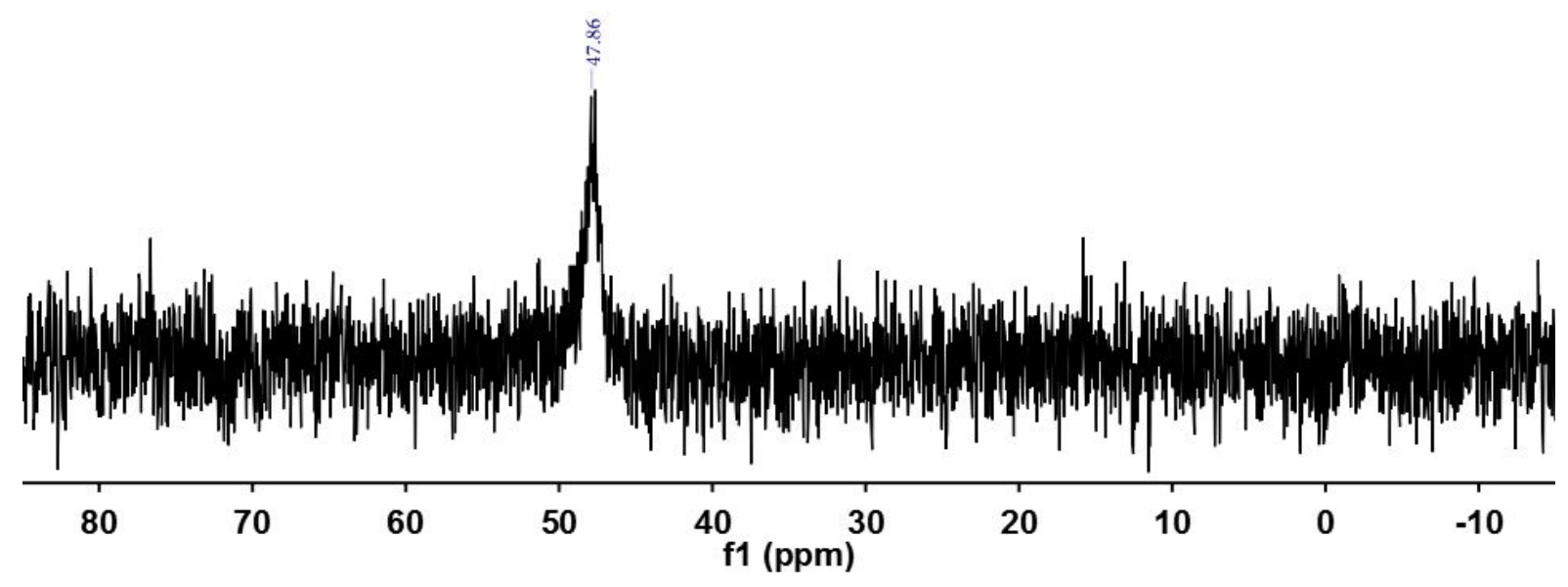

Figure S34. ${ }^{31} \mathrm{P}$ NMR spectra of the reaction of (a) 2a, (b) 2a with 0.1 equivalent of tert-butyl isocyanide, and (c) 2a with 0.1 equivalent of benzyl isocyanide in $\mathrm{C}_{6} \mathrm{D}_{6}$ at $60{ }^{\circ} \mathrm{C}$.

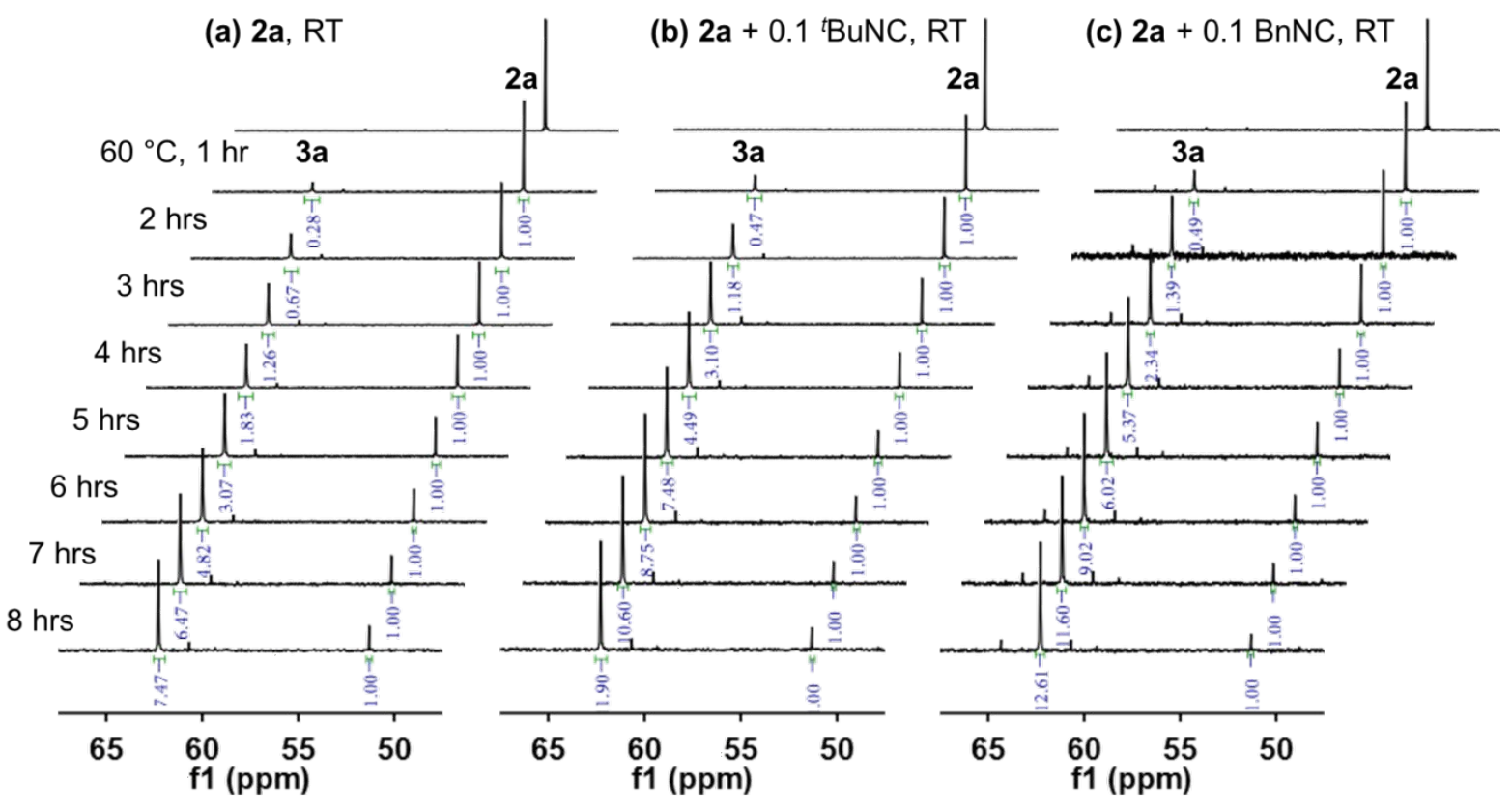


Figure S35. ${ }^{1} \mathrm{H}$ NMR spectrum $\left(\mathrm{C}_{6} \mathrm{D}_{6}, 400 \mathrm{MHz}\right)$ of $\left(\mathrm{PhSiP}_{2}\right) \mathrm{Ni}(\mathrm{OC}(\mathrm{O}) \mathrm{NHMes})(6 \mathbf{6})$ at room temperature.

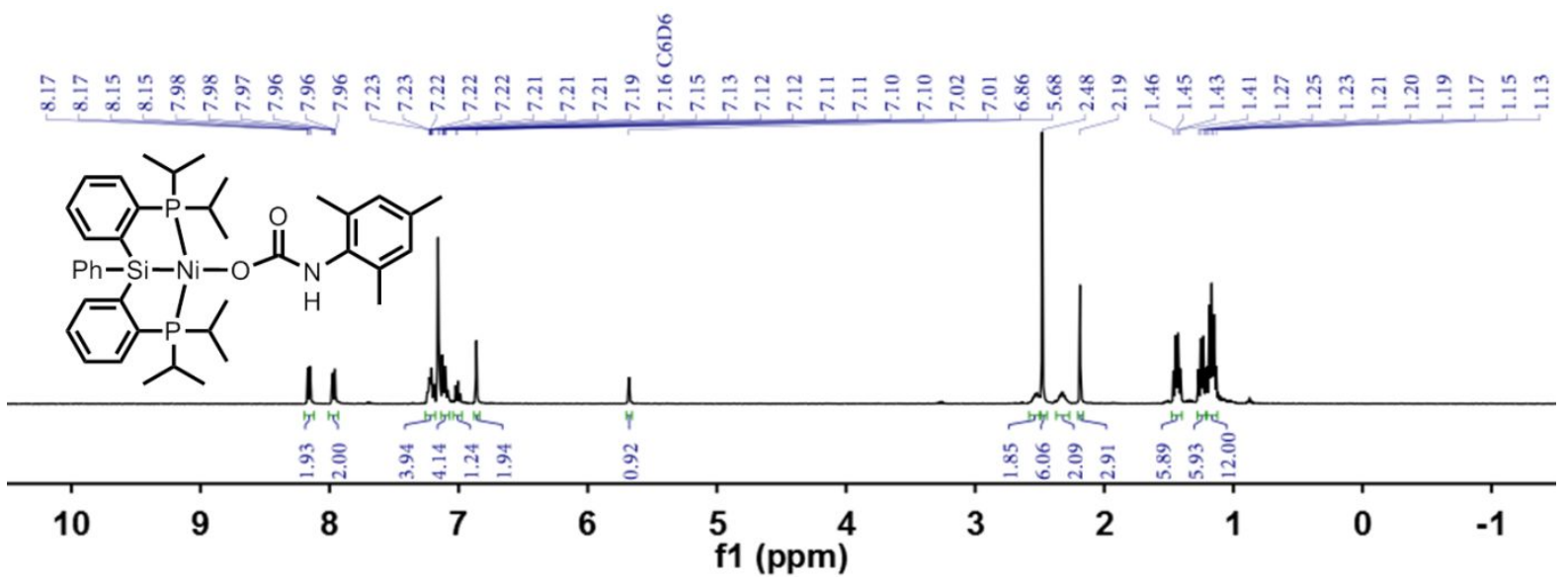

Figure S36. ${ }^{13} \mathrm{C}$ NMR spectrum $\left(\mathrm{C}_{6} \mathrm{D}_{6}, 101 \mathrm{MHz}\right)$ of $\left(\mathrm{PhSiP}_{2}\right) \mathrm{Ni}(\mathrm{OC}(\mathrm{O}) \mathrm{NHMes})(\mathbf{6 a})$ at room temperature.

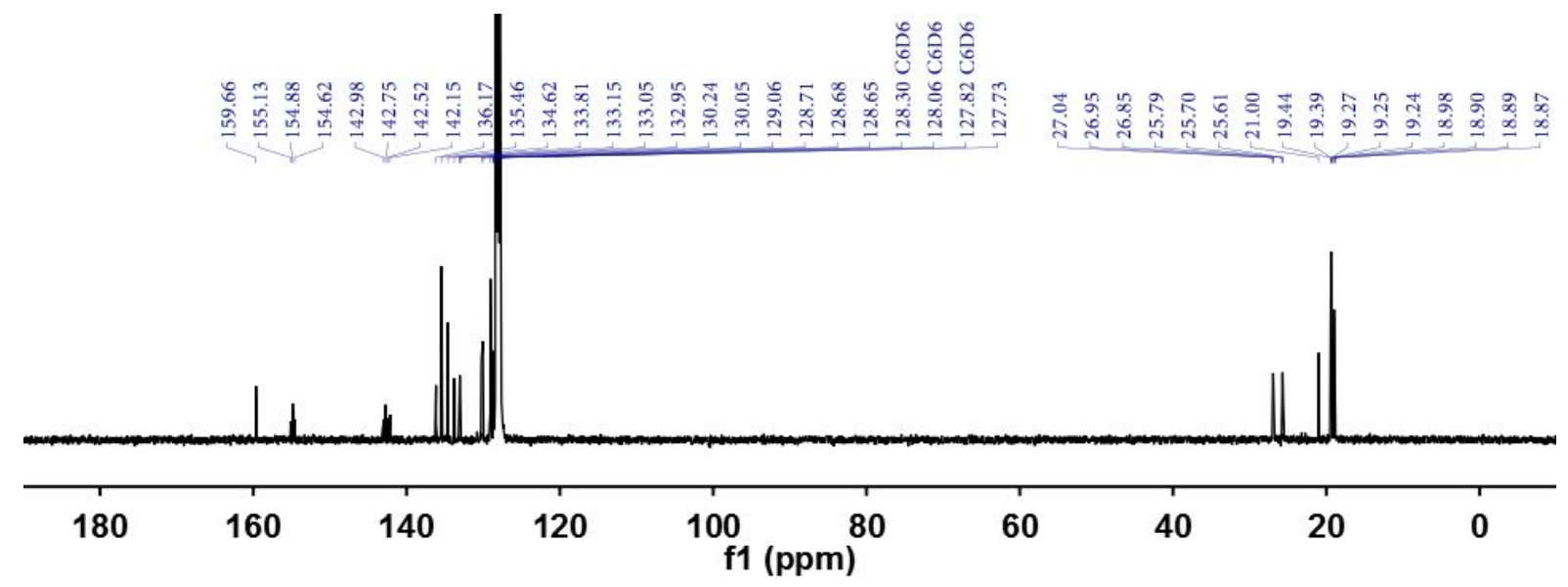

Figure S37. ${ }^{29} \mathrm{Si} \mathrm{NMR}$ spectrum $\left(\mathrm{C}_{6} \mathrm{D}_{6}, 79 \mathrm{MHz}\right)$ of $\left(\mathrm{PhSiP}_{2}\right) \mathrm{Ni}(\mathrm{OC}(\mathrm{O}) \mathrm{NHMes})(\mathbf{6 a})$ at room temperature.

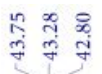

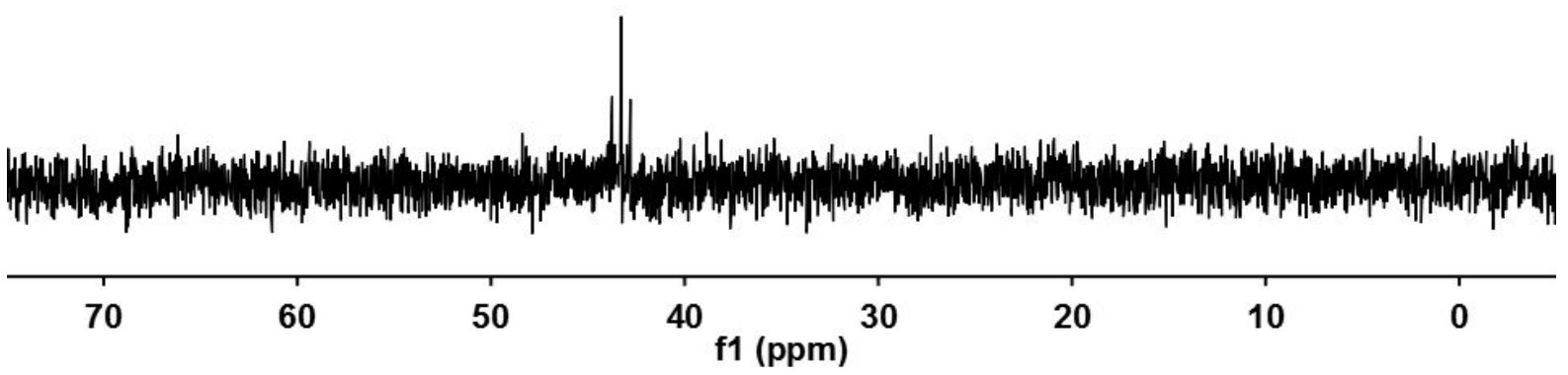


Figure S38. ${ }^{31} \mathrm{P}$ NMR spectrum $\left(\mathrm{C}_{6} \mathrm{D}_{6}, 162 \mathrm{MHz}\right)$ of $\left(\mathrm{PhSiP}_{2}\right) \mathrm{Ni}(\mathrm{OC}(\mathrm{O}) \mathrm{NHMes})(\mathbf{6 a})$ at room temperature.

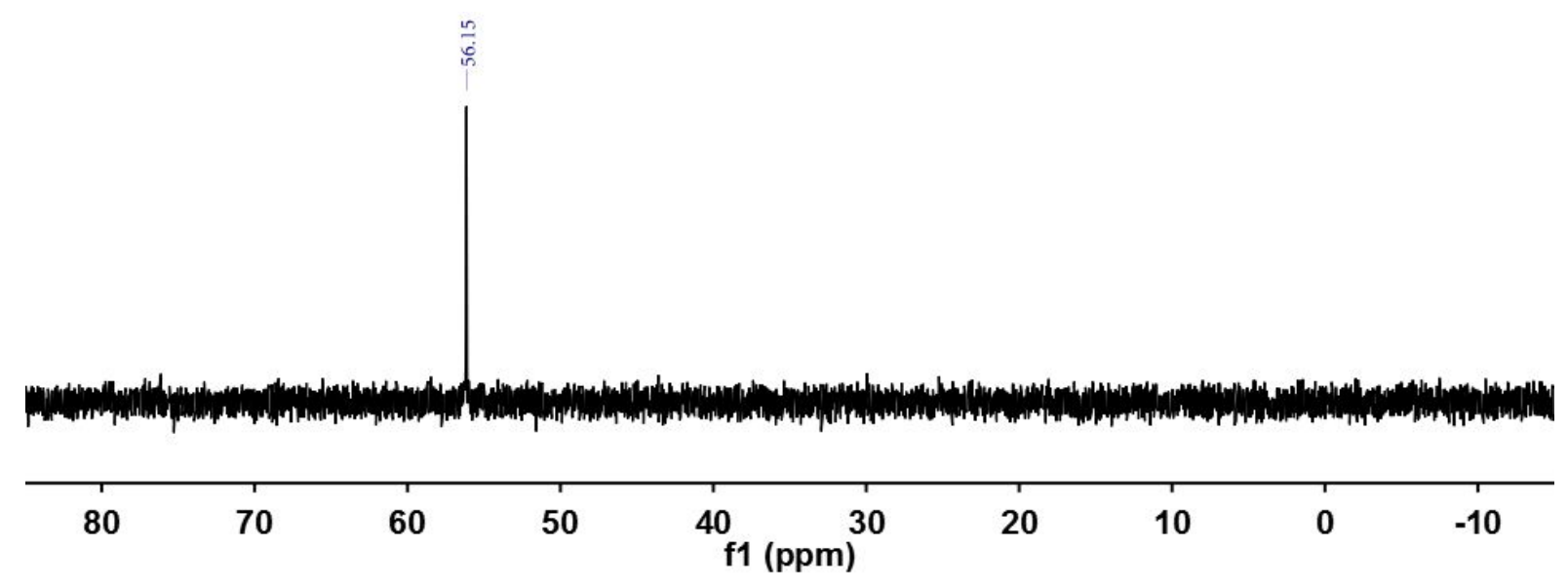

Figure S39. ${ }^{1} \mathrm{H}$ NMR spectrum $\left(\mathrm{C}_{6} \mathrm{D}_{6}, 400 \mathrm{MHz}\right)$ of $\left(\mathrm{PhSiP}_{2}\right) \mathrm{Ni}(\mathrm{OC}(\mathrm{O}) \mathrm{NHTrip})(6 \mathbf{b})$ at room temperature.
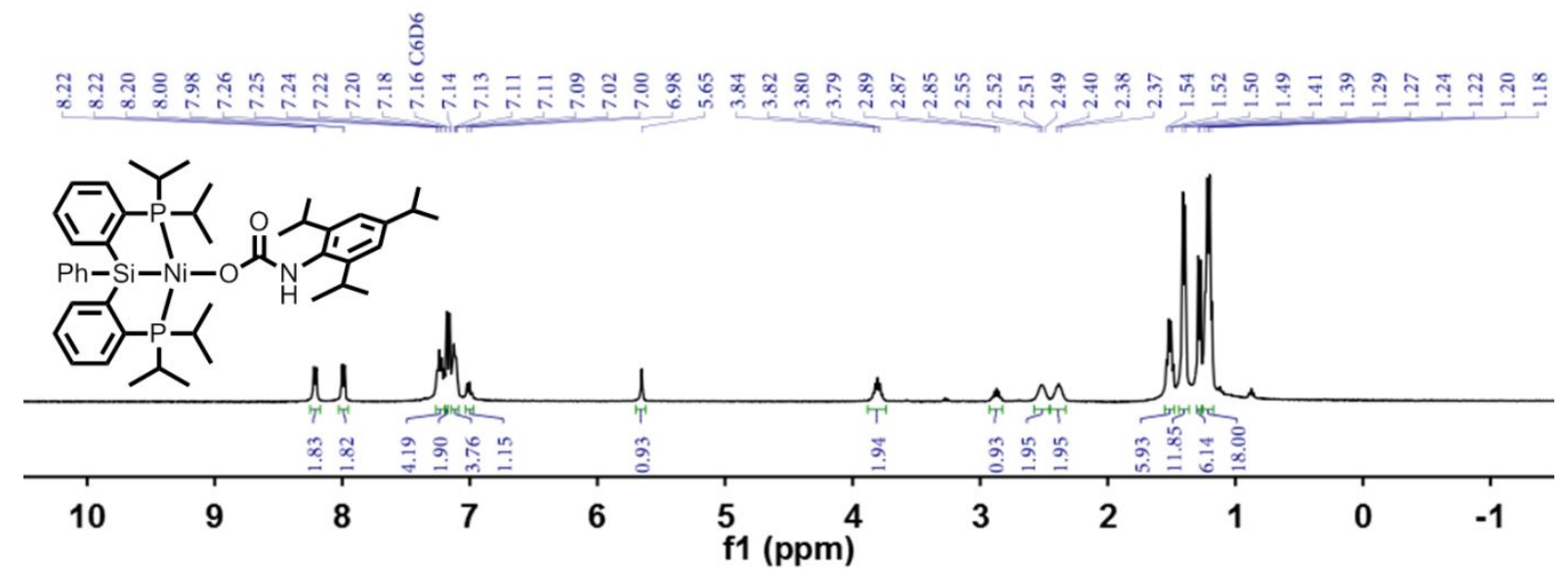

Figure S40. ${ }^{13} \mathrm{C}$ NMR spectrum $\left(\mathrm{C}_{6} \mathrm{D}_{6}, 101 \mathrm{MHz}\right)$ of $\left(\mathrm{PhSiP}_{2}\right) \mathrm{Ni}(\mathrm{OC}(\mathrm{O}) \mathrm{NHTrip})(\mathbf{6 b})$ at room temperature.

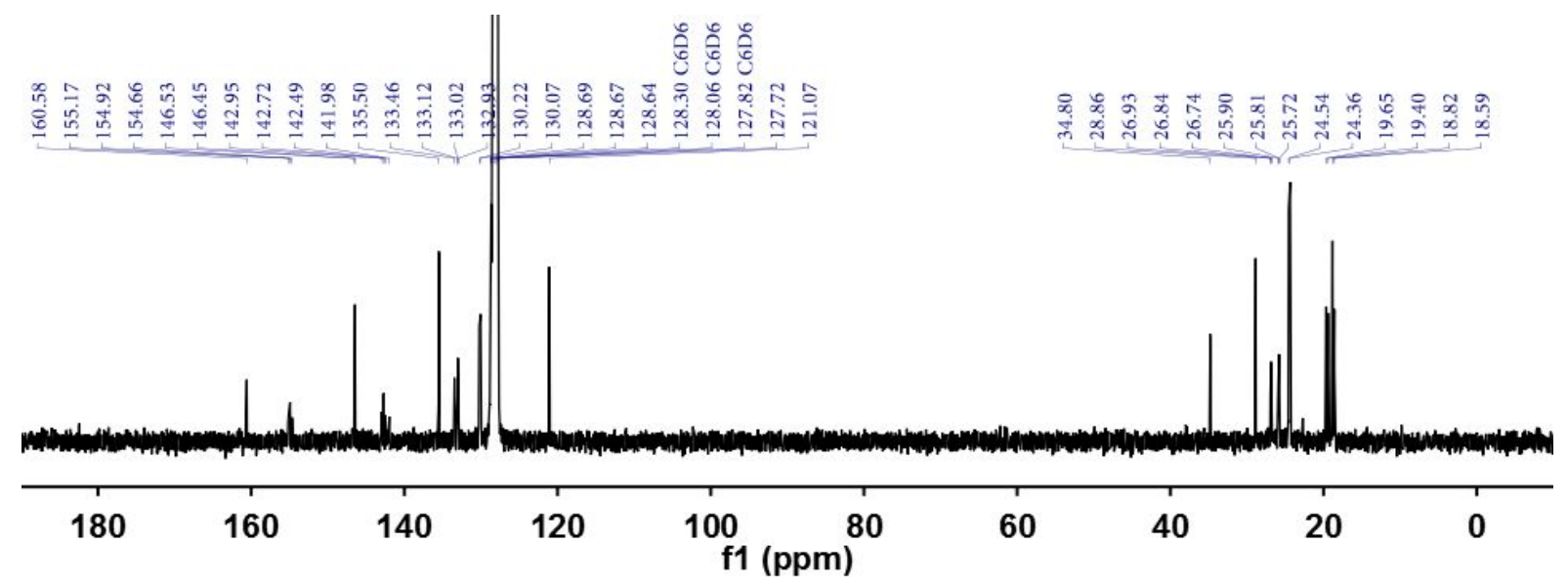


Figure S41. ${ }^{29} \mathrm{Si} \mathrm{NMR}$ spectrum $\left(\mathrm{C}_{6} \mathrm{D}_{6}, 79 \mathrm{MHz}\right)$ of $\left(\mathrm{PhSiP}_{2}\right) \mathrm{Ni}(\mathrm{OC}(\mathrm{O}) \mathrm{NHTrip})(\mathbf{6 b})$ at room temperature.

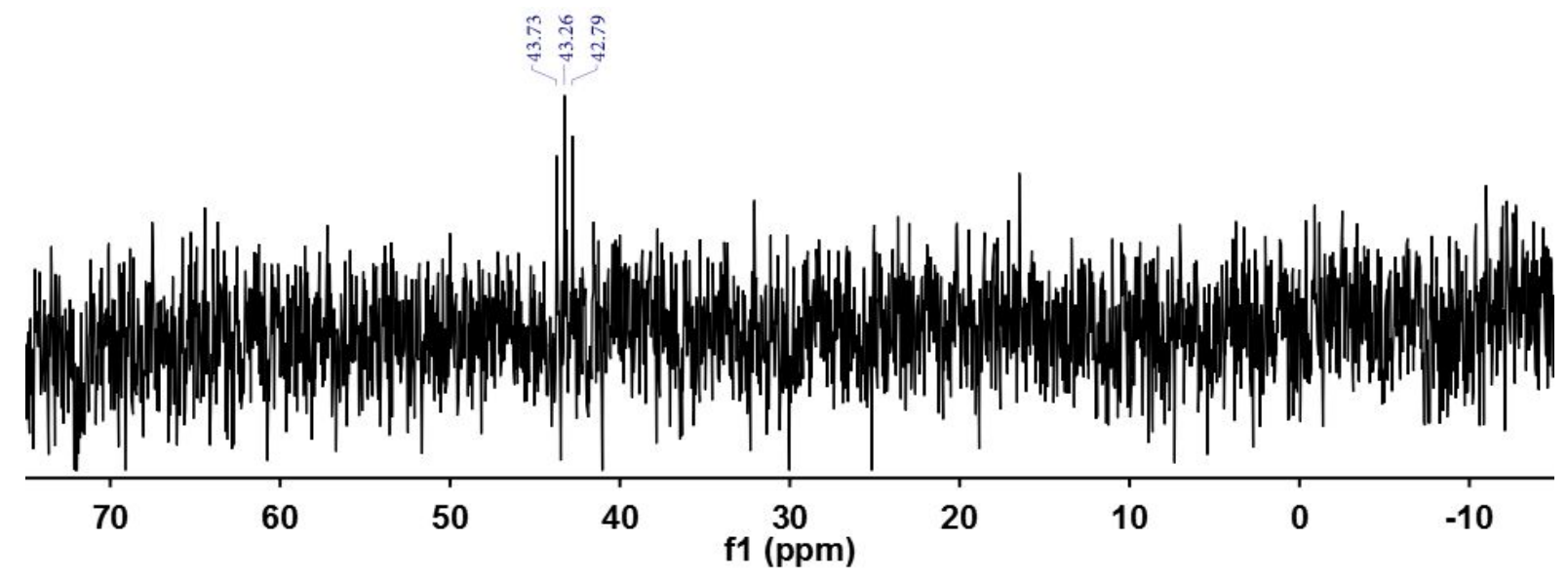

Figure S42. ${ }^{31} \mathrm{P}$ NMR spectrum $\left(\mathrm{C}_{6} \mathrm{D}_{6}, 162 \mathrm{MHz}\right)$ of $\left(\mathrm{PhSiP}_{2}\right) \mathrm{Ni}(\mathrm{OC}(\mathrm{O}) \mathrm{NHTrip})(6 \mathbf{b})$ at room temperature.

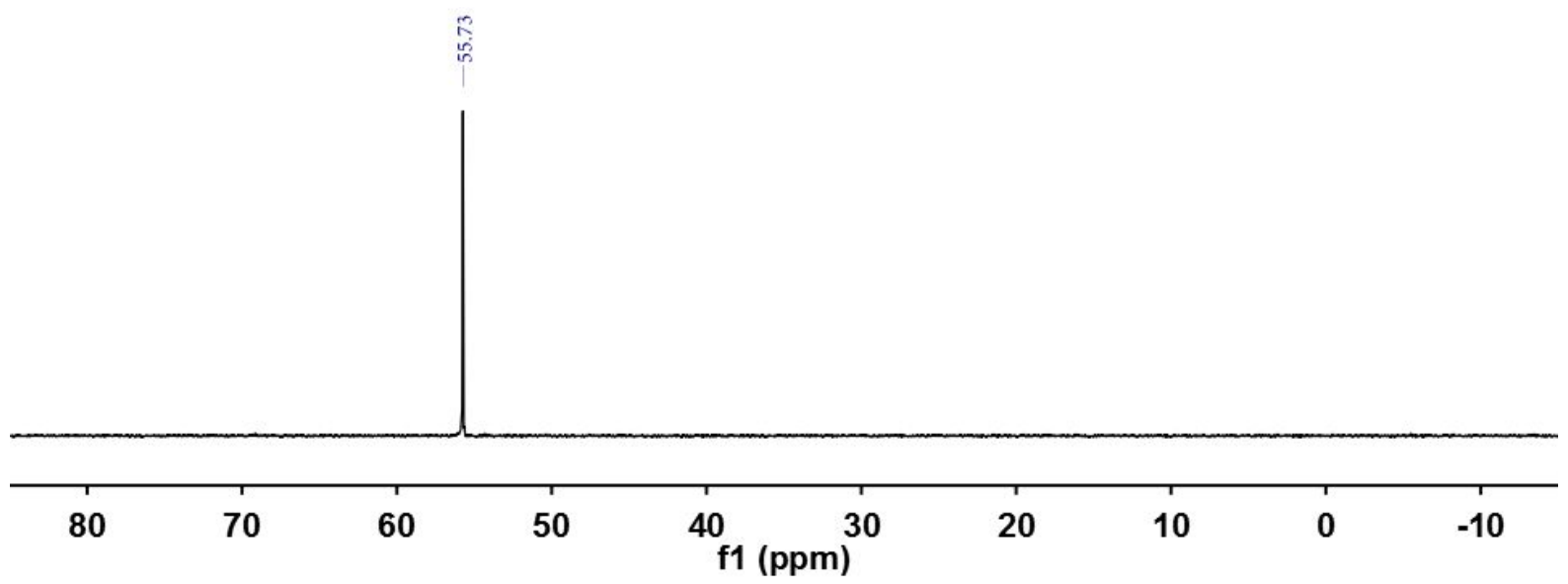


Figure S43. ${ }^{31} \mathrm{P}$ NMR spectra $\left(\mathrm{C}_{6} \mathrm{D}_{6}, 162 \mathrm{MHz}\right)$ of (a) the mixture of $\mathbf{3 a}$ and $\mathbf{6 a}$ generated from the reaction of the mixture of 2a and 3a with $\mathrm{CO}_{2}(\mathrm{~g})$, and (b) heating at $80{ }^{\circ} \mathrm{C}$ for $3 \mathrm{hr}$ after applying vacuum to generate $2 \mathbf{a}$.

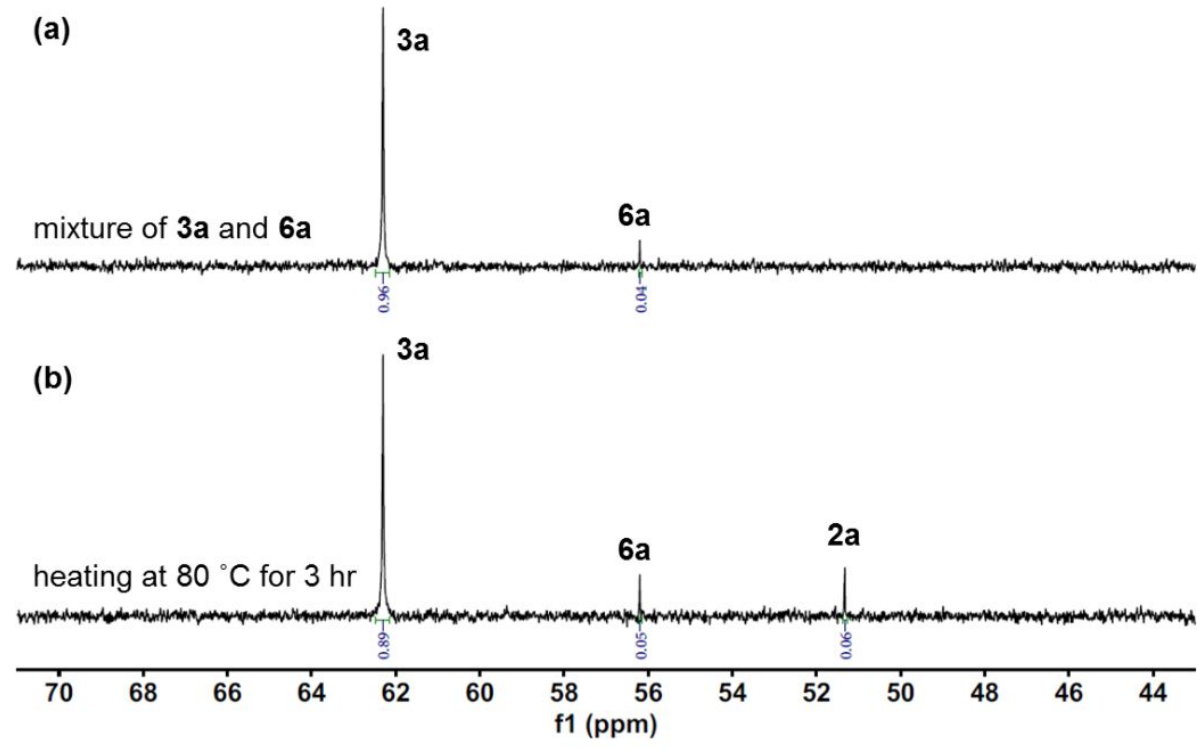

Figure S44. ${ }^{31} \mathrm{P}$ NMR spectra $\left(\mathrm{C}_{6} \mathrm{D}_{6}, 162 \mathrm{MHz}\right)$ of a mixture of $3 \mathbf{a}$ and $\mathbf{6 a}$ generated from the reaction of a mixture of $2 \mathbf{a}$ and $3 \mathbf{a}$ with $\mathrm{CO}_{2}(\mathrm{~g})$; excess $\mathrm{CO}_{2}$ was removed by applying vacuum and the mixture was heated at $80{ }^{\circ} \mathrm{C}$ for (a) $0,1,2$ and $3 \mathrm{hr}$ to generate $\mathbf{2 a}$ and (b) $0,1,2$ and $3 \mathrm{hr}$ under $\mathrm{CO}_{2}(\mathrm{~g})$ to generate $\mathbf{6 a}$.
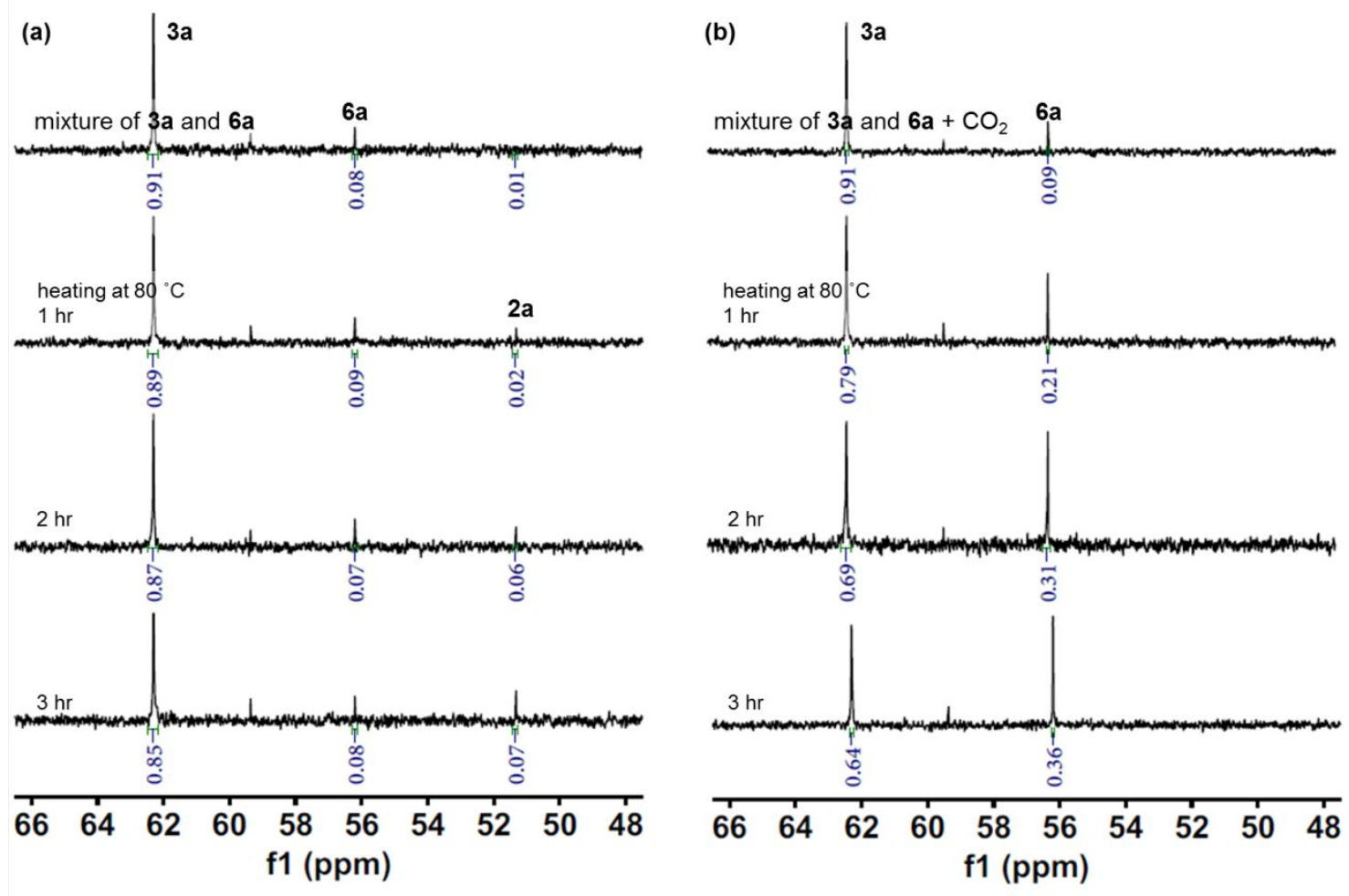
Figure S45. Solid state molecular structure of $\mathbf{1}$. All hydrogen atoms are omitted for clarity.

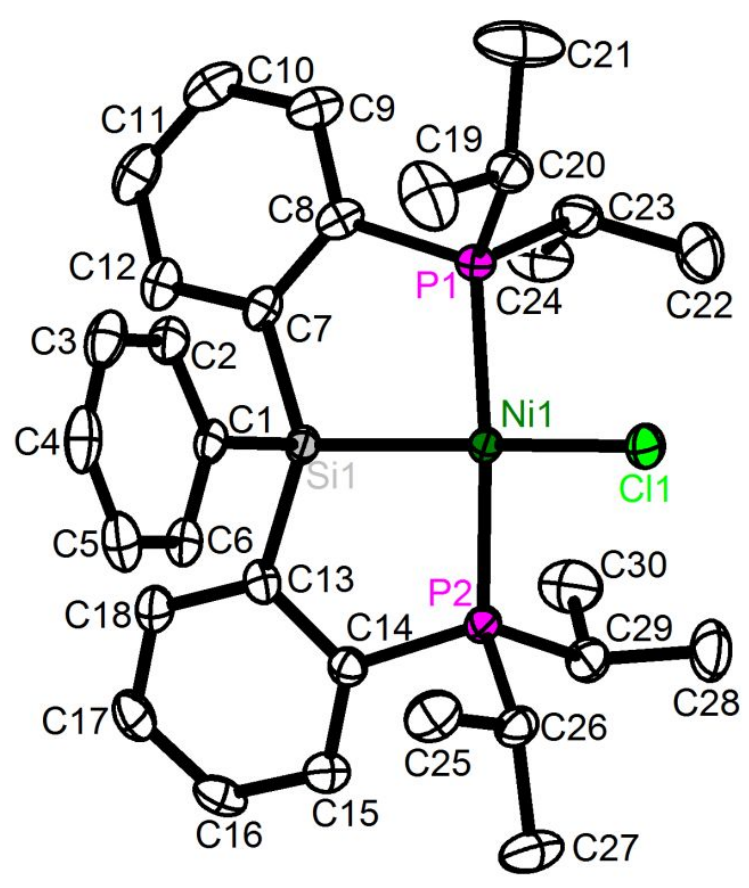

Table S1. Selected bond distances and angles for $\mathbf{1}\left(\AA\right.$ and $\left.^{\circ}\right)$.

\begin{tabular}{cccc}
\hline Distance & $\left(\mathrm{PhSiP}_{2}\right) \mathrm{NiCl}$ & Angle & $\left(\mathrm{PhSiP}_{2}\right) \mathrm{NiCl}$ \\
\hline $\mathrm{d}_{\mathrm{Ni1}-\mathrm{P} 1}$ & $2.1828(6)$ & $\angle \mathrm{P} 1-\mathrm{Ni1}-\mathrm{P} 2$ & $154.12(3)$ \\
\hline $\mathrm{d}_{\mathrm{Ni1}-\mathrm{P} 2}$ & $2.1848(6)$ & $\angle \mathrm{P} 1-\mathrm{Ni1}-\mathrm{Cl1}$ & $98.26(2)$ \\
& & $\angle \mathrm{P} 2-\mathrm{Ni1}-\mathrm{Cl1}$ & $98.72(2)$ \\
\hline $\mathrm{d}_{\mathrm{Ni1}-\mathrm{Cl1}}$ & $2.2270(6)$ & $\angle \mathrm{Si} 1-\mathrm{Ni1}-\mathrm{Cl1}$ & $159.68(3)$ \\
\hline $\mathrm{d}_{\mathrm{Ni1}-\mathrm{Si} 1}$ & $2.2044(6)$ & $\angle \mathrm{Si1}-\mathrm{Ni1}-\mathrm{P} 1$ & $84.73(2)$ \\
\hline
\end{tabular}


Figure S46. Solid state molecular structure of 2a. All hydrogen atoms except the N-H and C37-Hs are omitted for clarity. The $\mathrm{N}-\mathrm{H}$ hydrogen atom was located in the Fourier difference map and its position was freely refined.

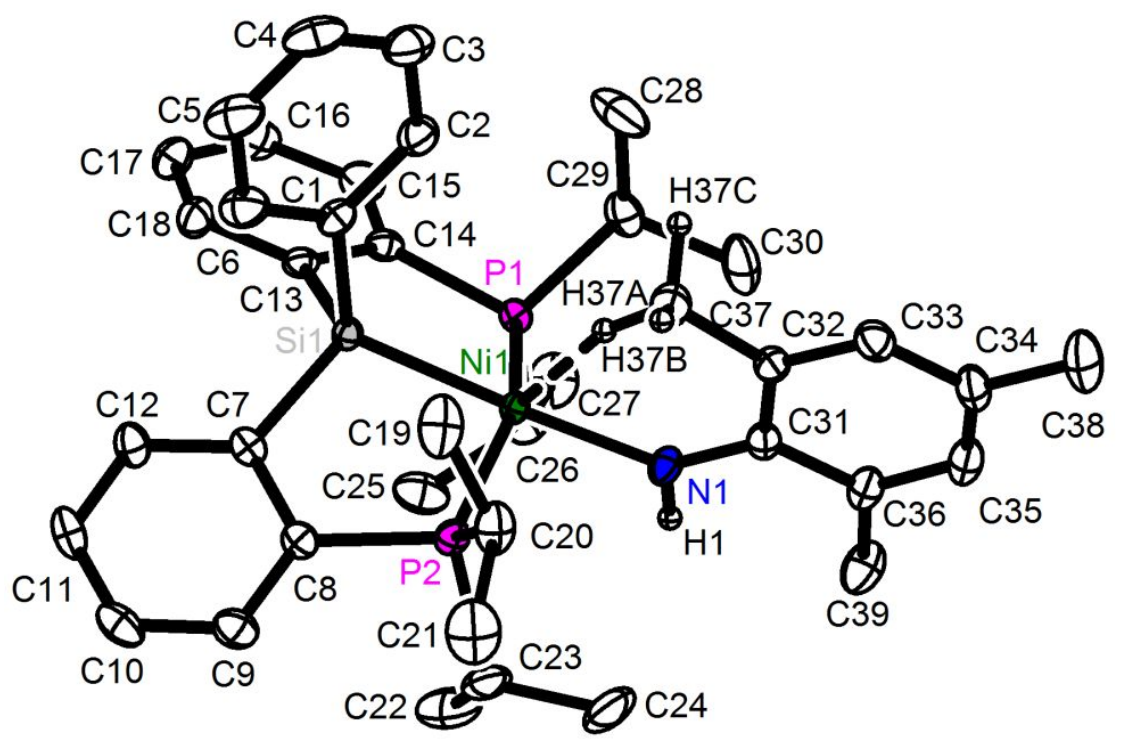

Table S2. Selected bond distances and angles for $\mathbf{2 a}\left(\AA\right.$ and $\left.^{\circ}\right)$.

\begin{tabular}{|c|c|c|c|}
\hline Distance & $\left(\mathrm{PhSiP}_{2}\right) \mathrm{Ni}(\mathrm{NHMes})$ & Angle & $\left(\mathrm{PhSiP}_{2}\right) \mathrm{Ni}(\mathrm{NHMes})$ \\
\hline $\mathrm{d}_{\mathrm{Ni1}-\mathrm{P} 1}$ & $2.1935(6)$ & $\angle \mathrm{P} 1-\mathrm{Ni} 1-\mathrm{P} 2$ & $153.41(2)$ \\
\hline \multirow{2}{*}{$\mathrm{d}_{\mathrm{Ni1}-\mathrm{P} 2}$} & \multirow{2}{*}{$2.2083(6)$} & $\angle \mathrm{P} 1-\mathrm{Ni1}-\mathrm{N} 1$ & $93.75(6)$ \\
\hline & & $\angle \mathrm{P} 2-\mathrm{Ni1}-\mathrm{N} 1$ & $98.29(6)$ \\
\hline $\mathrm{d}_{\mathrm{Ni1}-\mathrm{N} 1}$ & $1.960(2)$ & $\angle \mathrm{Si} 1-\mathrm{Ni1}-\mathrm{N} 1$ & $177.17(6)$ \\
\hline \multirow{2}{*}{$\mathrm{d}_{\mathrm{Ni1}-\mathrm{Si} 1}$} & \multirow{2}{*}{$2.2204(6)$} & $\angle \mathrm{Si} 1-\mathrm{Ni1}-\mathrm{P} 1$ & $84.69(2)$ \\
\hline & & $\angle \mathrm{Si} 1-\mathrm{Ni} 1-\mathrm{P} 2$ & $84.10(2)$ \\
\hline $\mathrm{d}_{\mathrm{Ni1-C} 37}$ & $2.962(2)$ & \multirow{2}{*}{$\angle \mathrm{Ni1}-\mathrm{H} 37 \mathrm{~B}-\mathrm{C} 37$} & \multirow{2}{*}{158.2} \\
\hline $\mathrm{d}_{\mathrm{Ni1}-\mathrm{H} 37 \mathrm{~B}}$ & 2.0294 & & \\
\hline
\end{tabular}


Figure S47. Solid state molecular structure of $\mathbf{3 b}$. Co-crystallized solvent molecule and all hydrogen atoms except the one at $\mathrm{N}$ are omitted for clarity. The $\mathrm{N}-\mathrm{H}$ hydrogen atom was located in the Fourier difference map and its position was freely refined.

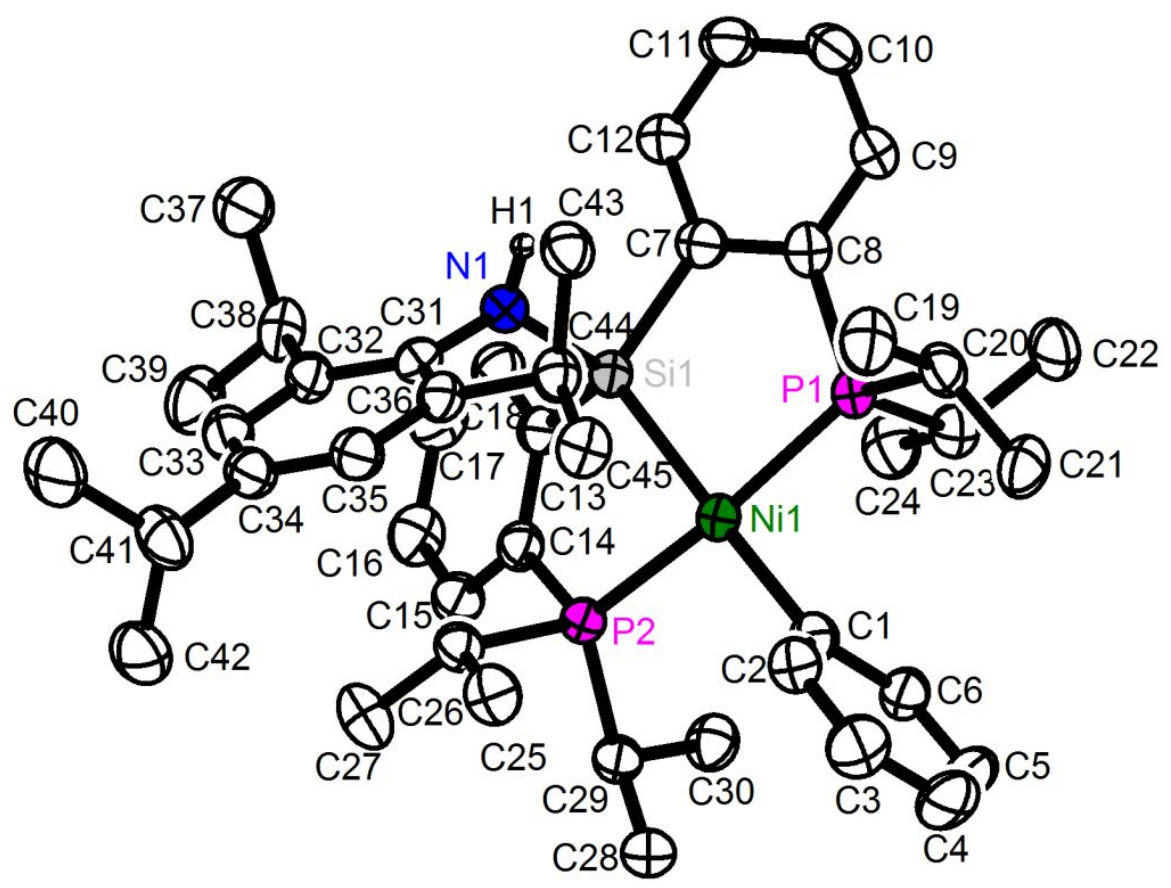

Table S3. Selected bond distances and angles for $\mathbf{3 b}\left(\AA\right.$ and $\left.^{\circ}\right)$.

\begin{tabular}{|c|c|c|c|}
\hline Distance & $\left((\right.$ TripNH$\left.) \mathrm{SiP}_{2}\right) \mathrm{Ni}(\mathrm{Ph})$ & Angle & $\left((\right.$ TripNH$\left.) \mathrm{SiP}_{2}\right) \mathrm{Ni}(\mathrm{Ph})$ \\
\hline $\mathrm{d}_{\mathrm{Ni1}-\mathrm{P} 1}$ & $2.172(1)$ & $\angle \mathrm{P} 1-\mathrm{Ni} 1-\mathrm{P} 2$ & $157.88(4)$ \\
\hline \multirow{2}{*}{$\mathrm{d}_{\mathrm{Ni1}-\mathrm{P} 2}$} & \multirow{2}{*}{$2.180(1)$} & $\angle \mathrm{P} 1-\mathrm{Ni1}-\mathrm{C} 1$ & $93.3(1)$ \\
\hline & & $\angle \mathrm{P} 2-\mathrm{Ni1}-\mathrm{C} 1$ & $99.8(1)$ \\
\hline $\mathrm{d}_{\mathrm{Ni1}-\mathrm{C} 1}$ & $1.974(4)$ & $\angle \mathrm{Si} 1-\mathrm{Ni} 1-\mathrm{C} 1$ & $176.1(1)$ \\
\hline \multirow{2}{*}{$\mathrm{d}_{\mathrm{Ni1}-\mathrm{Si} 1}$} & \multirow{2}{*}{$2.258(1)$} & $\angle \mathrm{Si} 1-\mathrm{Ni1}-\mathrm{P} 1$ & $84.73(4)$ \\
\hline & & $\angle \mathrm{Si} 1-\mathrm{Ni} 1-\mathrm{P} 2$ & $83.14(4)$ \\
\hline $\mathrm{d}_{\mathrm{Si1}-\mathrm{N} 1}$ & $1.766(3)$ & & \\
\hline
\end{tabular}


Figure S48. Solid state molecular structure of 4a. All hydrogen atoms except the one at $\mathrm{N}$ are omitted for clarity. The $\mathrm{N}-\mathrm{H}$ hydrogen atom was located in the Fourier difference map and its position was freely refined.

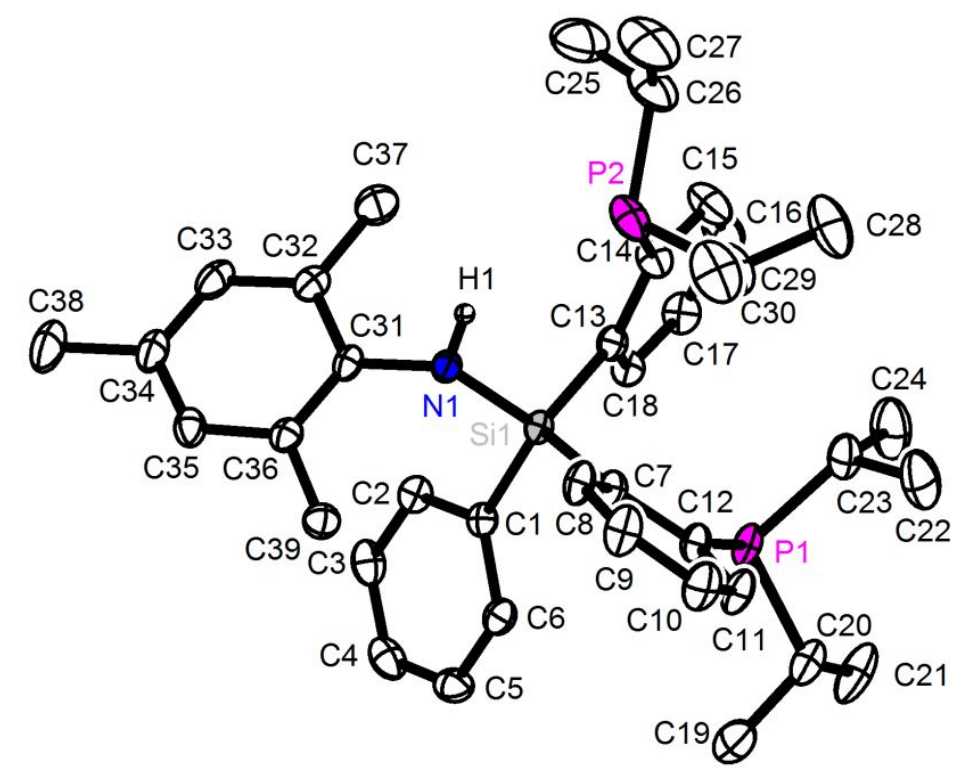

Table S4. Selected bond distances and angles for $4 \mathbf{a}\left(\AA\right.$ and $\left.^{\circ}\right)$.

\begin{tabular}{|c|c|c|c|}
\hline Distance & $(\mathrm{MesNH}) \mathrm{PhSiP}_{2}$ & Angle & $(\mathrm{MesNH}) \mathrm{PhSiP}_{2}$ \\
\hline $\mathrm{d}_{\mathrm{Si1-N1}}$ & $1.744(2)$ & $\angle \mathrm{C} 1-\mathrm{Si} 1-\mathrm{N} 1$ & $106.51(8)$ \\
\hline \multirow{2}{*}{$\mathrm{d}_{\mathrm{Si1-C1}}$} & \multirow{2}{*}{$1.871(2)$} & $\angle \mathrm{C} 1-\mathrm{Si} 1-\mathrm{C} 7$ & $108.99(8)$ \\
\hline & & $\angle \mathrm{C} 1-\mathrm{Si} 1-\mathrm{C} 13$ & $110.40(8)$ \\
\hline \multirow{2}{*}{$\mathrm{d}_{\mathrm{Si1-C7}}$} & \multirow{2}{*}{$1.900(2)$} & $\angle \mathrm{C} 7-\mathrm{Si} 1-\mathrm{N} 1$ & $108.73(8)$ \\
\hline & & $\angle \mathrm{C} 7-\mathrm{Si} 1-\mathrm{C} 13$ & 111.91(8) \\
\hline $\mathrm{d}_{\mathrm{Si} 1-\mathrm{C} 13}$ & $1.899(2)$ & $\angle \mathrm{C} 13-\mathrm{Si} 1-\mathrm{N} 1$ & $110.13(8)$ \\
\hline
\end{tabular}


Figure S49. Solid state molecular structure of $\mathbf{4 b}$. All hydrogen atoms except the one at $\mathrm{N}$ are omitted for clarity. The $\mathrm{N}-\mathrm{H}$ hydrogen atom was located in the Fourier difference map and its position was freely refined.

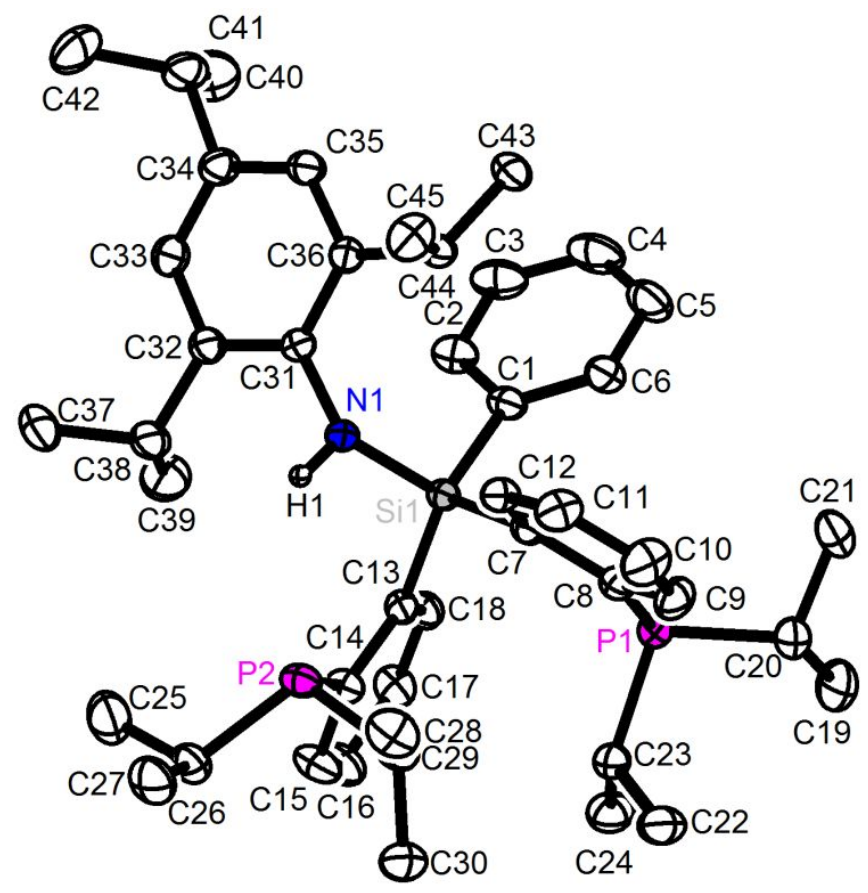

Table S5. Selected bond distances and angles for $\mathbf{4 b}\left(\AA\right.$ and $\left.^{\circ}\right)$.

\begin{tabular}{|c|c|c|c|}
\hline Distance & $($ TripNH)PhSiP 2 & Angle & $($ TripNH)PhSiP 2 \\
\hline $\mathrm{d}_{\mathrm{Si1-N} 1}$ & $1.734(1)$ & $\angle \mathrm{C} 1-\mathrm{Si} 1-\mathrm{N} 1$ & $106.81(7)$ \\
\hline \multirow{2}{*}{$\mathrm{d}_{\mathrm{Si1-C1}}$} & \multirow{2}{*}{$1.886(2)$} & $\angle \mathrm{C} 1-\mathrm{Si} 1-\mathrm{C} 7$ & $109.69(7)$ \\
\hline & & $\angle \mathrm{C} 1-\mathrm{Si} 1-\mathrm{C} 13$ & $111.14(7)$ \\
\hline \multirow{2}{*}{$\mathrm{d}_{\mathrm{Si} 1-\mathrm{C} 7}$} & \multirow{2}{*}{$1.896(2)$} & $\angle \mathrm{C} 7-\mathrm{Si} 1-\mathrm{N} 1$ & $107.33(7)$ \\
\hline & & $\angle \mathrm{C} 7-\mathrm{Si} 1-\mathrm{C} 13$ & $112.19(7)$ \\
\hline $\mathrm{d}_{\mathrm{Si} 1-\mathrm{C} 13}$ & $1.899(2)$ & $\angle \mathrm{C} 13-\mathrm{Si} 1-\mathrm{N} 1$ & $109.47(6)$ \\
\hline
\end{tabular}


Figure S50. Solid state molecular structure of 5. All hydrogen atoms except the one at $\mathrm{N}$ are omitted for clarity. The $\mathrm{N}-\mathrm{H}$ hydrogen atom was located in the Fourier difference map and its position was freely refined.

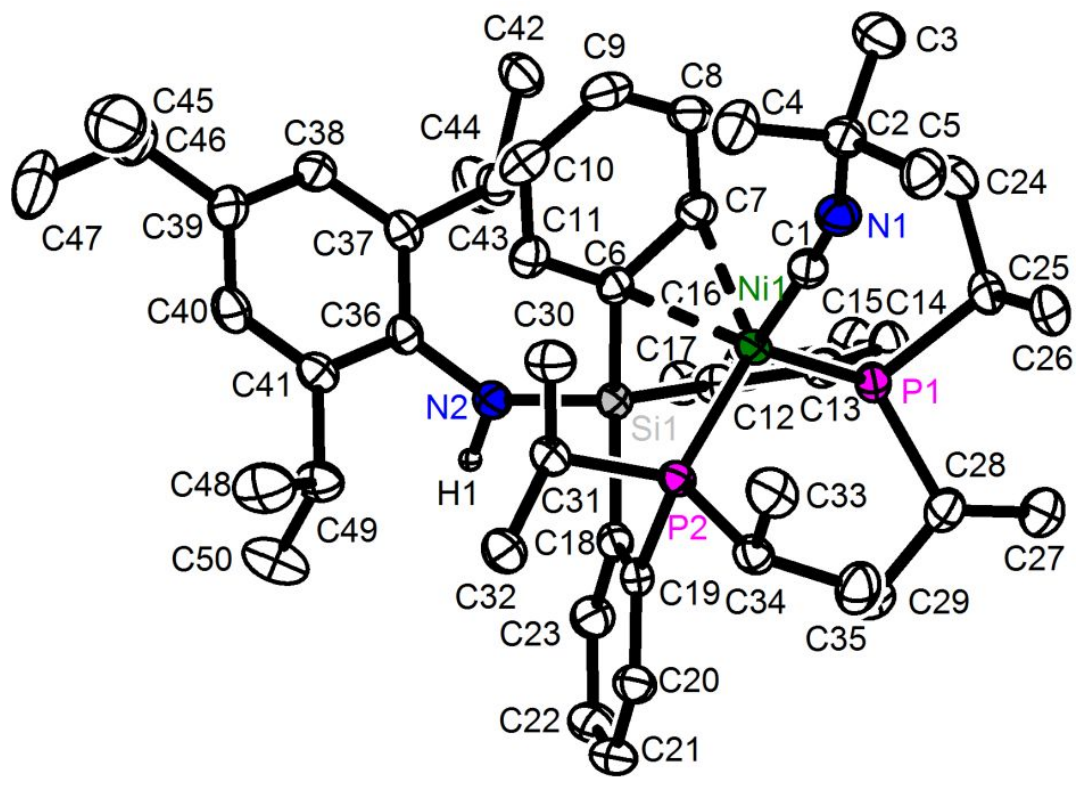

Table S6. Selected bond distances and angles for $\mathbf{5}\left(\AA\right.$ and $\left.^{\circ}\right)$.

\begin{tabular}{|c|c|c|c|}
\hline Distance & $\left((\operatorname{TripNH})\left(\eta^{2}-\mathrm{Ph}\right) \mathrm{SiP}_{2}\right) \mathrm{Ni}\left(\mathrm{CN}^{t} \mathrm{Bu}\right)$ & Angle & $\left((\operatorname{TripNH})\left(\eta^{2}-\mathrm{Ph}\right) \mathrm{SiP}_{2}\right) \mathrm{Ni}\left(\mathrm{CN}^{t} \mathrm{Bu}\right)$ \\
\hline $\mathrm{d}_{\mathrm{Ni1}-\mathrm{P} 1}$ & $2.2273(7)$ & \multirow{2}{*}{$\angle \mathrm{P} 1-\mathrm{Ni} 1-\mathrm{P} 2$} & \multirow{2}{*}{$117.62(2)$} \\
\hline $\mathrm{d}_{\mathrm{N} 1-\mathrm{P} 2}$ & $2.1990(6)$ & & \\
\hline \multirow{2}{*}{$\mathrm{d}_{\mathrm{Ni1}-\mathrm{Si} 1}$} & \multirow{2}{*}{$2.8741(7)$} & $\angle \mathrm{P} 1-\mathrm{Ni1}-\mathrm{C} 1$ & $111.37(7)$ \\
\hline & & $\angle \mathrm{P} 2-\mathrm{Ni} 1-\mathrm{C} 1$ & $105.10(7)$ \\
\hline \multirow{2}{*}{$\mathrm{d}_{\mathrm{Ni1}-\mathrm{C} 1}$} & \multirow{2}{*}{$1.834(2)$} & $\angle \mathrm{P} 1-\mathrm{Ni1}-\mathrm{C} 6$ & $107.92(6)$ \\
\hline & & $\angle \mathrm{P} 2-\mathrm{Ni1}-\mathrm{C} 6$ & $97.39(6)$ \\
\hline $\mathrm{d}_{\mathrm{Ni} 1-\mathrm{C} 6}$ & $2.205(2)$ & $\angle \mathrm{P} 1-\mathrm{Ni1}-\mathrm{C} 7$ & $91.98(7)$ \\
\hline $\mathrm{d}_{\mathrm{Ni} 1-\mathrm{C} 7}$ & $2.223(2)$ & $\angle \mathrm{P} 2-\mathrm{Ni1}-\mathrm{C} 7$ & $134.20(6)$ \\
\hline \multirow{2}{*}{$\mathrm{d}_{\mathrm{C} 1-\mathrm{N} 1}$} & \multirow{2}{*}{$1.173(3)$} & $\angle \mathrm{C} 1-\mathrm{Ni} 1-\mathrm{C} 6$ & $117.12(9)$ \\
\hline & & $\angle \mathrm{C} 1-\mathrm{Ni} 1-\mathrm{C} 7$ & $93.92(9)$ \\
\hline $\mathrm{d}_{\mathrm{C} 6-\mathrm{C} 7}$ & $1.432(3)$ & $\angle \mathrm{Si} 1-\mathrm{Ni} 1-\mathrm{P} 1$ & $82.15(2)$ \\
\hline $\mathrm{d}_{\mathrm{C} 6-\mathrm{C} 11}$ & $1.432(3)$ & $\angle \mathrm{Si} 1-\mathrm{Ni} 1-\mathrm{P} 2$ & $82.76(2)$ \\
\hline $\mathrm{d}_{\mathrm{C} 7-\mathrm{C} 8}$ & $1.420(3)$ & $\angle \mathrm{Si} 1-\mathrm{Ni} 1-\mathrm{C} 1$ & $157.30(7)$ \\
\hline $\mathrm{d}_{\mathrm{C} 8-\mathrm{C} 9}$ & $1.366(4)$ & \multirow{3}{*}{$\begin{array}{l}\angle \mathrm{Sil}-\mathrm{Ni1}-\mathrm{C} 6 \\
\angle \mathrm{Si} 1-\mathrm{Ni1}-\mathrm{C} 7\end{array}$} & \multirow{3}{*}{$\begin{array}{l}40.19(6) \\
66.65(6)\end{array}$} \\
\hline $\mathrm{d}_{\mathrm{C} 9-\mathrm{C} 10}$ & $1.408(4)$ & & \\
\hline $\mathrm{d}_{\mathrm{C} 10-\mathrm{C} 11}$ & $1.371(3)$ & & \\
\hline
\end{tabular}


Figure S51. Solid state molecular structure of 6a. Co-crystallized solvent molecule and all hydrogen atoms except the one at $\mathrm{N}$ are omitted for clarity. The $\mathrm{N}-\mathrm{H}$ hydrogen atom was located in the Fourier difference map and its position was freely refined.

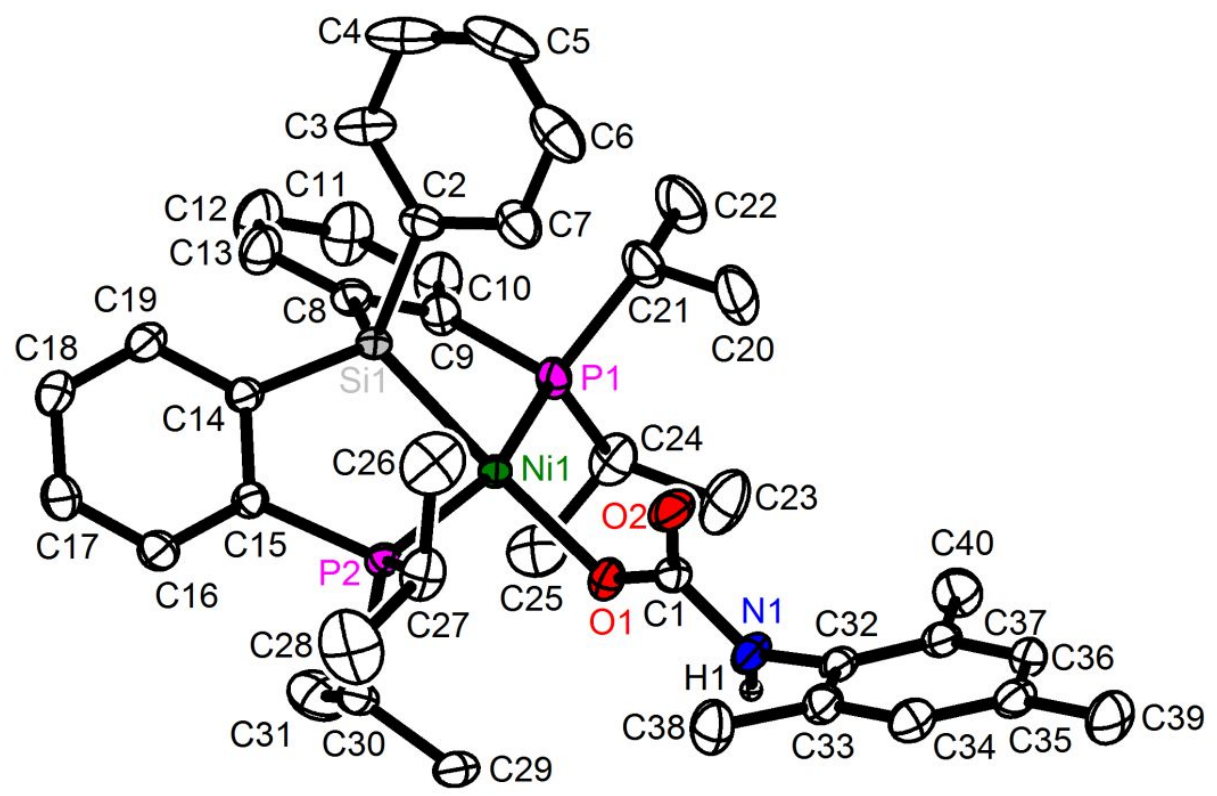

Table S7. Selected bond distances and angles for $\mathbf{6 a}\left(\AA\right.$ and $\left.^{\circ}\right)$.

\begin{tabular}{|c|c|c|c|}
\hline Distance & $\left(\mathrm{PhSiP}_{2}\right) \mathrm{Ni}(\mathrm{OC}(\mathrm{O}) \mathrm{NHMes})$ & Angle & $\left(\mathrm{PhSiP}_{2}\right) \mathrm{Ni}(\mathrm{OC}(\mathrm{O}) \mathrm{NHMes})$ \\
\hline $\mathrm{d}_{\mathrm{Ni} 1-\mathrm{P} 1}$ & $2.1956(4)$ & $\angle \mathrm{P} 1-\mathrm{Ni1}-\mathrm{P} 2$ & $158.84(2)$ \\
\hline \multirow{2}{*}{$\mathrm{d}_{\mathrm{Ni1}-\mathrm{P} 2}$} & \multirow{2}{*}{$2.1845(4)$} & $\angle \mathrm{P} 1-\mathrm{Ni1}-\mathrm{O} 1$ & $96.16(3)$ \\
\hline & & $\angle \mathrm{P} 2-\mathrm{Ni1}-\mathrm{O} 1$ & $97.20(3)$ \\
\hline $\mathrm{d}_{\mathrm{Ni1-O1}}$ & $1.948(1)$ & \multirow{2}{*}{$\angle \mathrm{Si} 1-\mathrm{Ni} 1-\mathrm{O} 1$} & \multirow{2}{*}{$177.09(4)$} \\
\hline $\mathrm{d}_{\mathrm{Ni1}-\mathrm{O} 2}$ & $2.938(2)$ & & \\
\hline \multirow{2}{*}{$\mathrm{d}_{\mathrm{Ni1}-\mathrm{Si} 1}$} & \multirow{2}{*}{$2.2103(4)$} & $\angle \mathrm{Si} 1-\mathrm{Ni1}-\mathrm{P} 1$ & $82.79(2)$ \\
\hline & & $\angle \mathrm{Si} 1-\mathrm{Ni1}-\mathrm{P} 2$ & $84.59(2)$ \\
\hline \multirow{2}{*}{$\mathrm{d}_{\mathrm{C} 1-\mathrm{O} 1}$} & \multirow{2}{*}{$1.288(2)$} & $\angle \mathrm{Ni1}-\mathrm{O} 1-\mathrm{C} 1$ & $114.7(2)$ \\
\hline & & $\angle \mathrm{Ni} 1-\mathrm{O} 2-\mathrm{C} 1$ & $68.91(9)$ \\
\hline \multirow{2}{*}{$\mathrm{d}_{\mathrm{C} 1-\mathrm{O} 2}$} & \multirow{2}{*}{$1.233(2)$} & $\angle \mathrm{O} 1-\mathrm{C} 1-\mathrm{O} 2$ & $126.4(1)$ \\
\hline & & $\angle \mathrm{O} 1-\mathrm{C} 1-\mathrm{N} 1$ & $112.3(1)$ \\
\hline
\end{tabular}


Figure S52. Solid state molecular structure of $6 \mathbf{b}$. All hydrogen atoms except the one at $\mathrm{N}$ are omitted for clarity. The N-H hydrogen atoms were located in the Fourier difference map and their positions were freely refined.
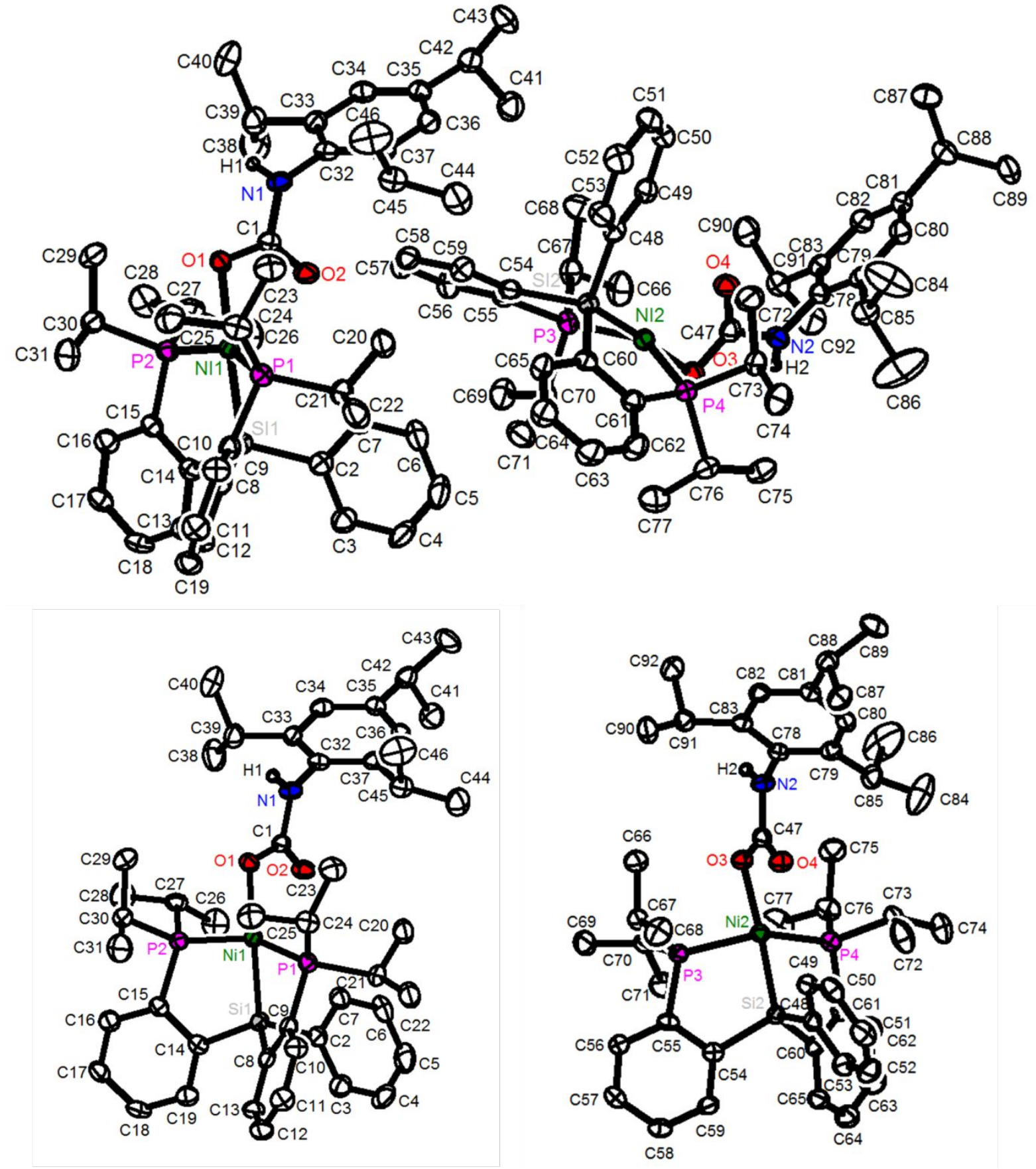
Table S8. Selected bond distances and angles for $\mathbf{6 b}\left(\AA\right.$ and $\left.^{\circ}\right)$.

\begin{tabular}{|c|c|c|c|c|c|}
\hline Distance & $\begin{array}{c}\left(\mathrm{PhSiP}_{2}\right) \mathrm{Ni} \\
(\mathrm{OC}(\mathrm{O}) \mathrm{NHTrip})\end{array}$ & Angle & $\begin{array}{c}\left(\mathrm{PhSiP}_{2}\right) \mathrm{Ni} \\
(\mathrm{OC}(\mathrm{O}) \mathrm{NHTrip})\end{array}$ & Angle & $\begin{array}{c}\left(\mathrm{PhSiP}_{2}\right) \mathrm{Ni} \\
(\mathrm{OC}(\mathrm{O}) \mathrm{NHTrip})\end{array}$ \\
\hline $\mathrm{d}_{\mathrm{Ni1}-\mathrm{P} 1}$ & $2.2035(9)$ & $\angle \mathrm{P} 1-\mathrm{Ni} 1-\mathrm{P} 2$ & $153.66(4)$ & $\angle \mathrm{Si} 1-\mathrm{Ni} 1-\mathrm{P} 2$ & $84.95(3)$ \\
\hline $\mathrm{d}_{\mathrm{Ni} 2-\mathrm{P} 3}$ & $2.1773(9)$ & $\angle \mathrm{P} 3-\mathrm{Ni} 2-\mathrm{P} 4$ & $149.61(4)$ & $\angle \mathrm{Si} 2-\mathrm{Ni} 2-\mathrm{P} 4$ & $84.33(3)$ \\
\hline $\mathrm{d}_{\mathrm{Ni1}-\mathrm{P} 2}$ & $2.1831(9)$ & $\angle \mathrm{P} 1-\mathrm{Ni1}-\mathrm{O} 1$ & $99.31(6)$ & $\angle \mathrm{Ni1}-\mathrm{O} 1-\mathrm{C} 1$ & $101.4(2)$ \\
\hline $\mathrm{d}_{\mathrm{Ni2}-\mathrm{P} 4}$ & $2.2042(9)$ & $\angle \mathrm{P} 3-\mathrm{Ni} 2-\mathrm{O} 3$ & $93.21(6)$ & $\angle \mathrm{Ni} 2-\mathrm{O} 3-\mathrm{C} 47$ & $96.2(2)$ \\
\hline $\mathrm{d}_{\mathrm{Ni1}-\mathrm{O} 1}$ & $1.999(2)$ & $\angle \mathrm{P} 2-\mathrm{Ni1}-\mathrm{O} 1$ & $95.06(6)$ & $\angle \mathrm{Ni} 1-\mathrm{O} 2-\mathrm{C} 1$ & $77.2(2)$ \\
\hline $\mathrm{d}_{\mathrm{Ni2}-\mathrm{O} 3}$ & $2.031(2)$ & $\angle \mathrm{P} 4-\mathrm{Ni} 2-\mathrm{O} 3$ & $97.26(6)$ & $\angle \mathrm{Ni} 2-\mathrm{O} 4-\mathrm{C} 47$ & $80.4(2)$ \\
\hline$\overline{d_{\text {Ni1-O2 }}}$ & $2.554(2)$ & $\angle \mathrm{Si} 1-\mathrm{Ni1}-\mathrm{O} 1$ & $177.59(6)$ & $\angle \mathrm{O} 1-\mathrm{C} 1-\mathrm{O} 2$ & $124.2(3)$ \\
\hline $\mathrm{d}_{\mathrm{Ni} 2-\mathrm{O} 4}$ & $2.403(2)$ & $\angle \mathrm{Si} 2-\mathrm{Ni} 2-\mathrm{O} 3$ & $177.92(7)$ & $\angle \mathrm{O} 3-\mathrm{C} 47-\mathrm{O} 4$ & $123.7(3)$ \\
\hline $\mathrm{d}_{\mathrm{Ni1}-\mathrm{Si} 1}$ & $2.217(1)$ & $\angle \mathrm{Si} 1-\mathrm{Ni1}-\mathrm{P} 1$ & $81.60(3)$ & $\angle \mathrm{O} 1-\mathrm{C} 1-\mathrm{N} 1$ & $115.4(3)$ \\
\hline $\mathrm{d}_{\mathrm{Ni} 2-\mathrm{Si} 2}$ & $2.216(1)$ & $\angle \mathrm{Si} 2-\mathrm{Ni} 2-\mathrm{P} 3$ & $84.75(3)$ & $\angle \mathrm{O} 3-\mathrm{C} 47-\mathrm{N} 2$ & $116.0(3)$ \\
\hline $\mathrm{d}_{\mathrm{C} 1-\mathrm{O} 1}$ & $1.284(3)$ & & & & \\
\hline $\mathrm{d}_{\mathrm{C} 47-\mathrm{O} 3}$ & $1.280(4)$ & & & & \\
\hline$\overline{d_{\mathrm{C} 1-\mathrm{O} 2}}$ & $1.239(3)$ & & & & \\
\hline $\mathrm{d}_{\mathrm{C} 47-\mathrm{O} 4}$ & $1.248(4)$ & & & & \\
\hline
\end{tabular}


Figure S53. UV-Vis spectrum of $\mathbf{1}$ in THF at room temperature.

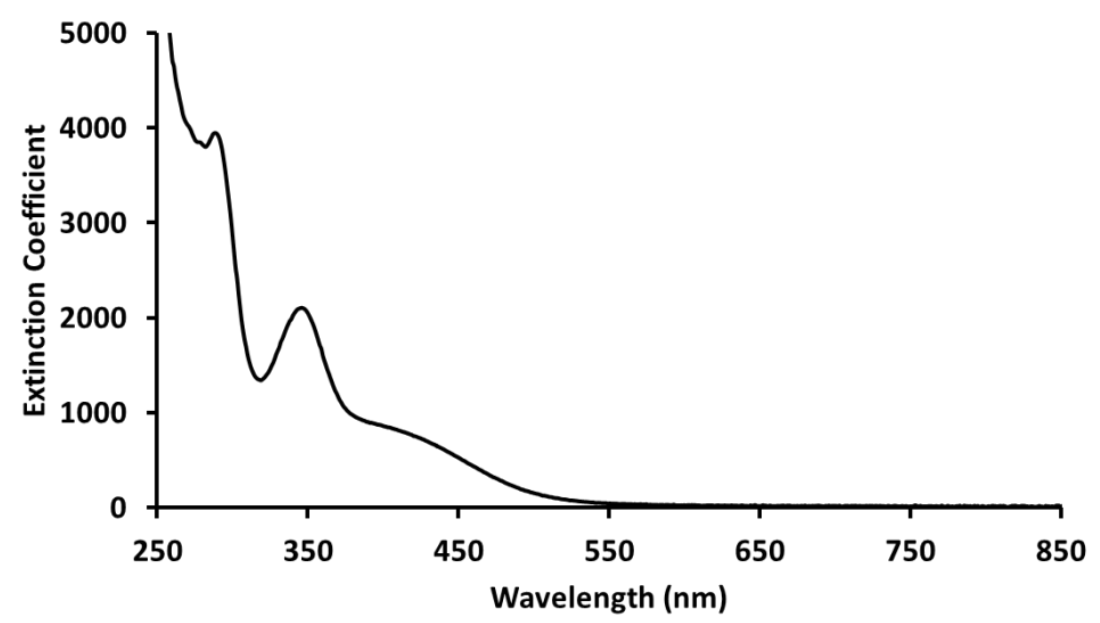

Figure S54. UV-Vis spectrum of $\mathbf{2 a}$ in THF at room temperature.

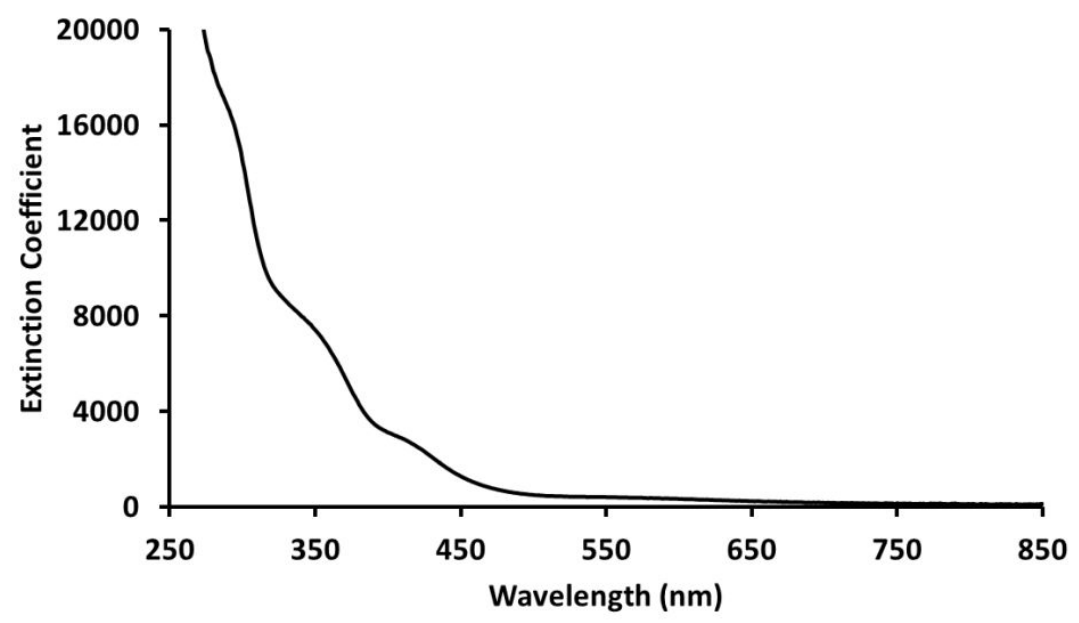

Figure $\mathbf{S 5 5}$. UV-Vis spectrum of $\mathbf{3 b}$ in THF at room temperature.

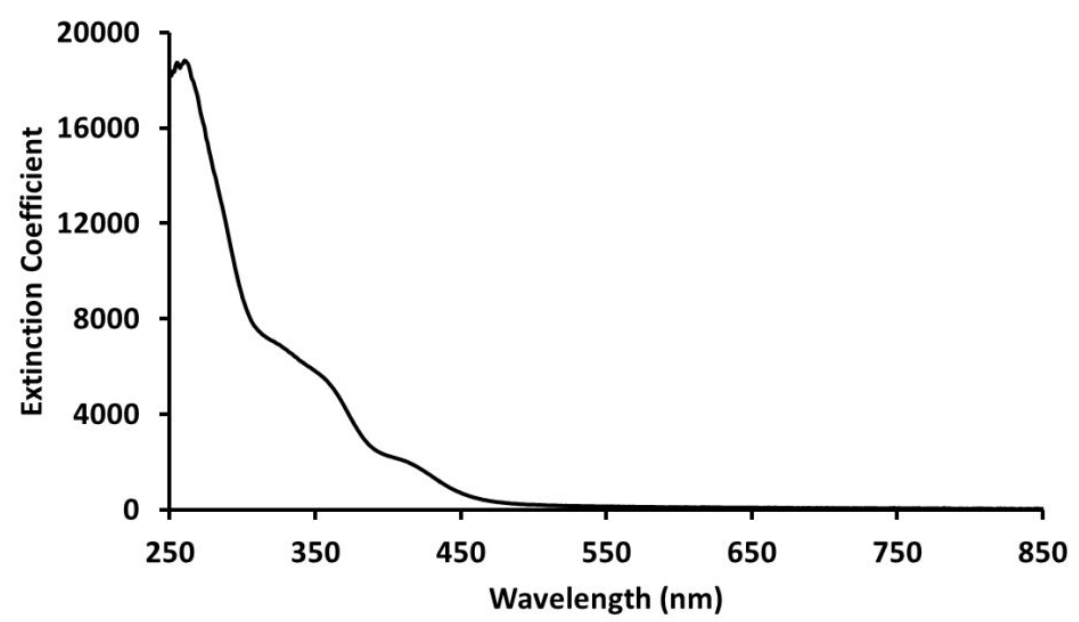


Figure S56. UV-Vis spectrum of 5 in THF at room temperature.

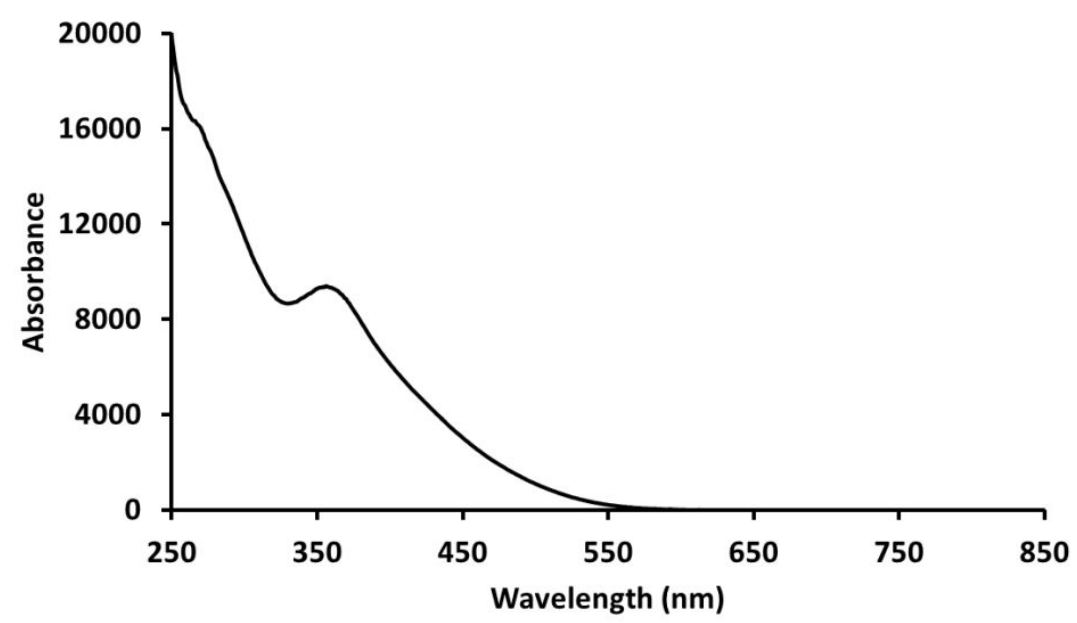

Figure S57. UV-Vis spectra of $\mathbf{6 a}$ in THF at room temperature.

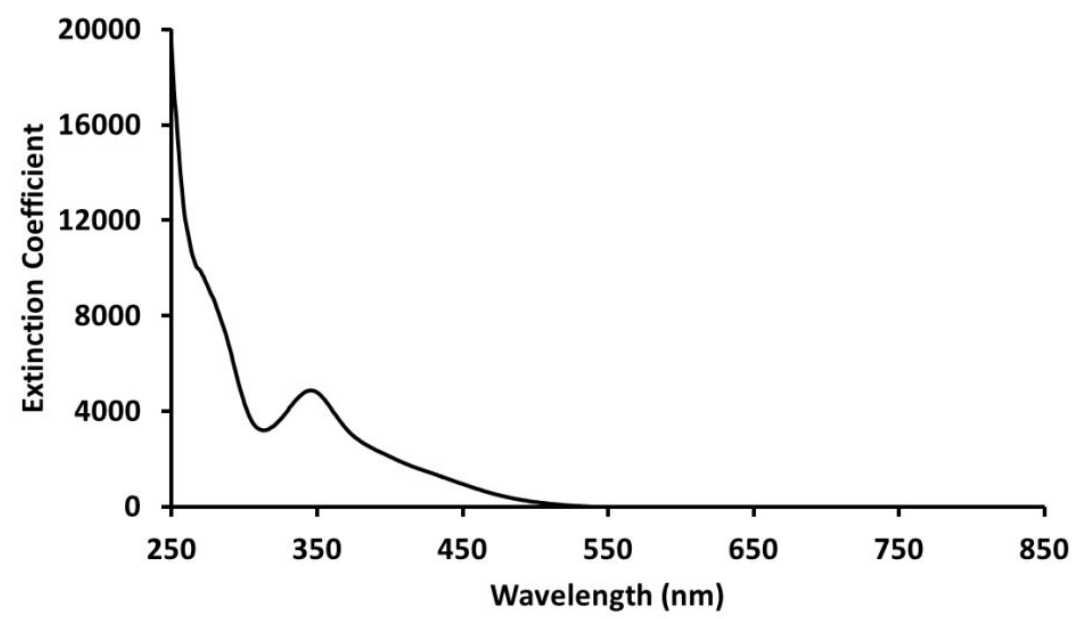

Figure S58. UV-Vis spectra of $\mathbf{6 b}$ in $\mathrm{THF}$ at room temperature.

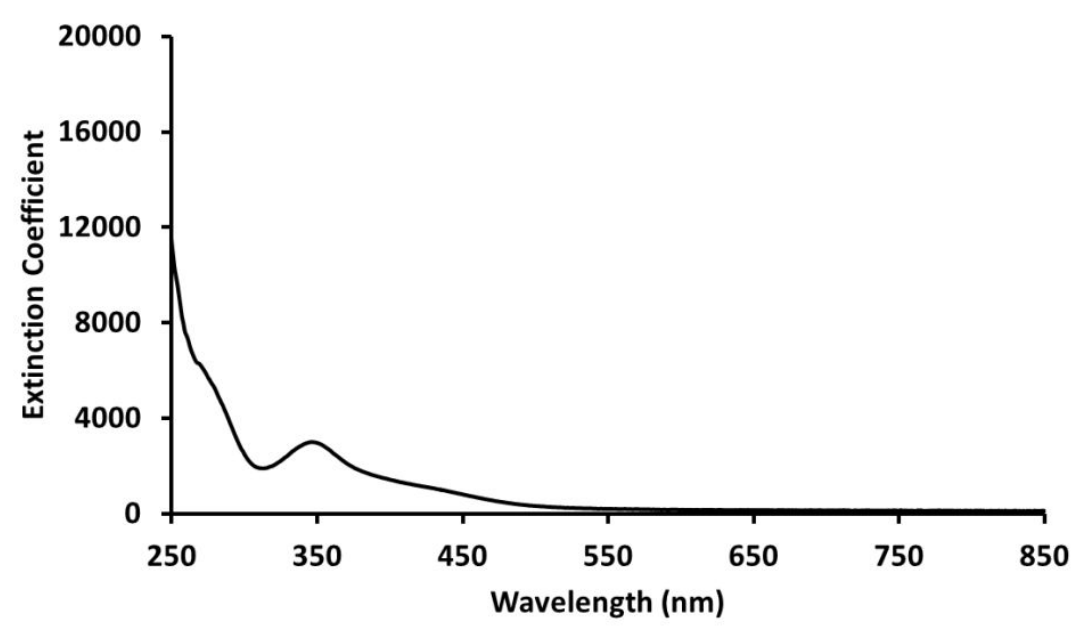


Figure S59. IR spectrum of $\mathbf{1}$ at room temperature. (KBr pellet)

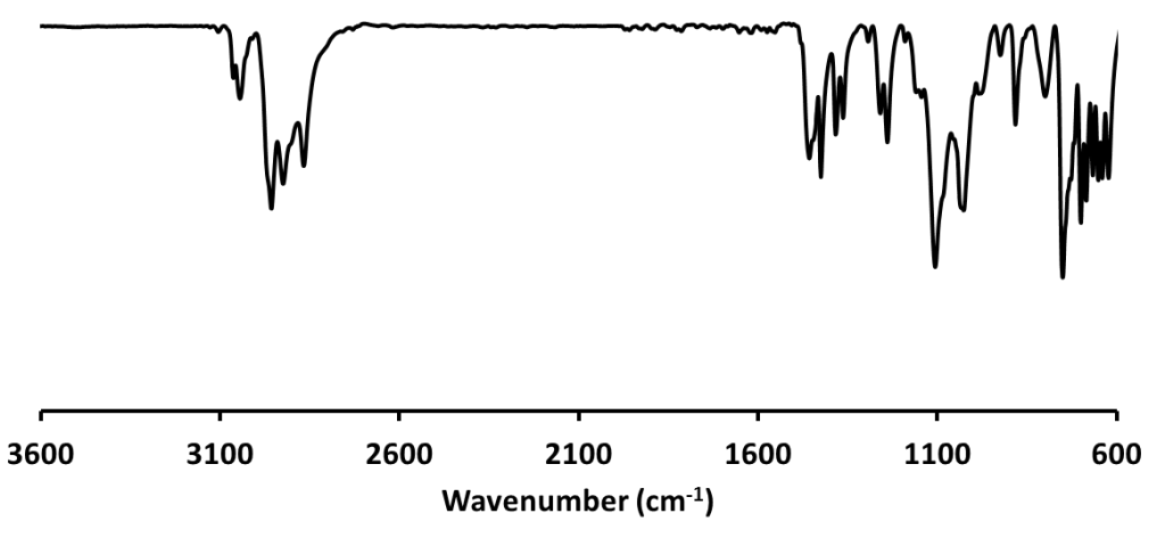

Figure S60. IR spectrum of $\mathbf{2 a}$ at room temperature. (KBr pellet)

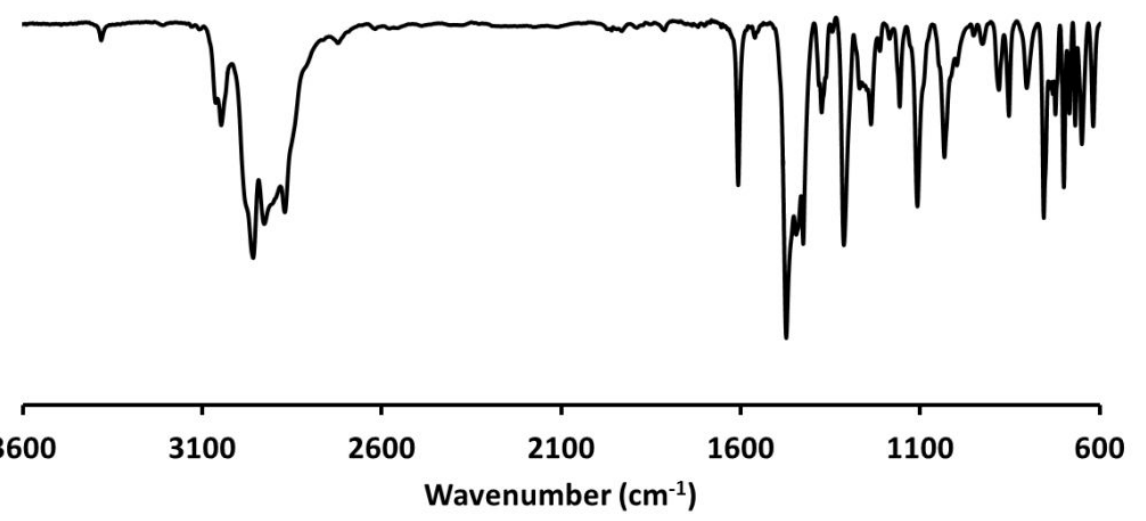

Figure S61. IR spectrum of $\mathbf{3 b}$ at room temperature. (KBr pellet)

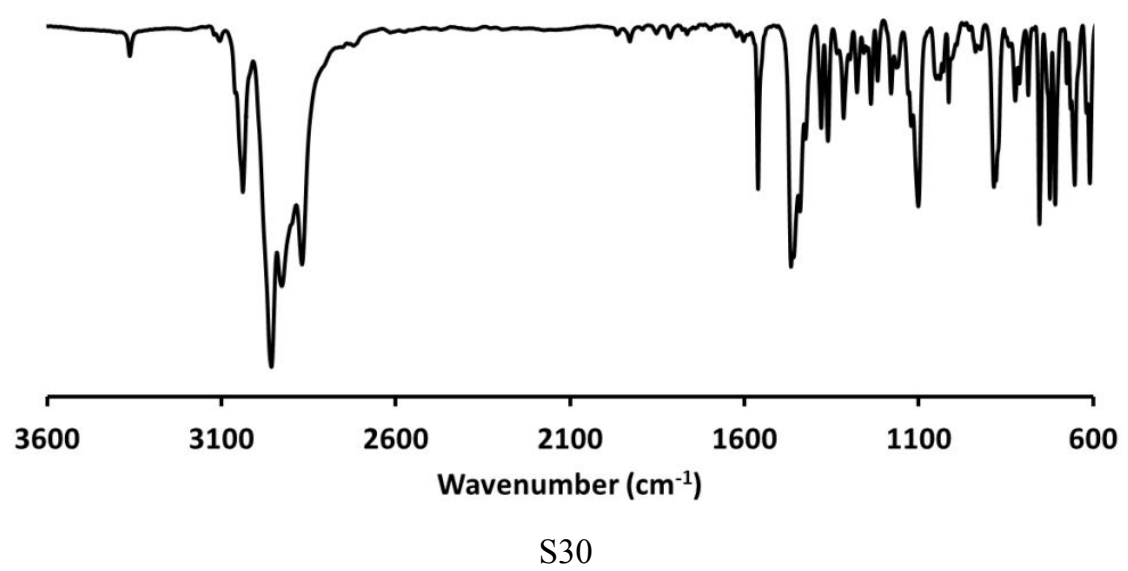


Figure S62. IR NMR spectrum of an a unknown species at room temperature, obtained from in situ reaction mixture of 1, 2,4,6-triisopropylaniline and NaHMDS in CO atmosphere. (KBr pellet)

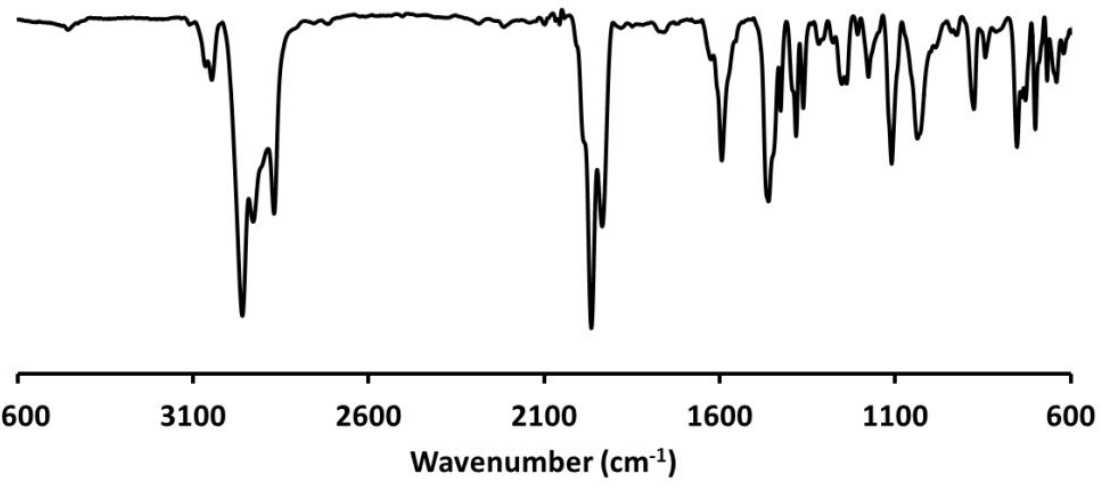

Figure S63. IR spectrum of $\mathbf{4 a}$ at room temperature. (KBr pellet)

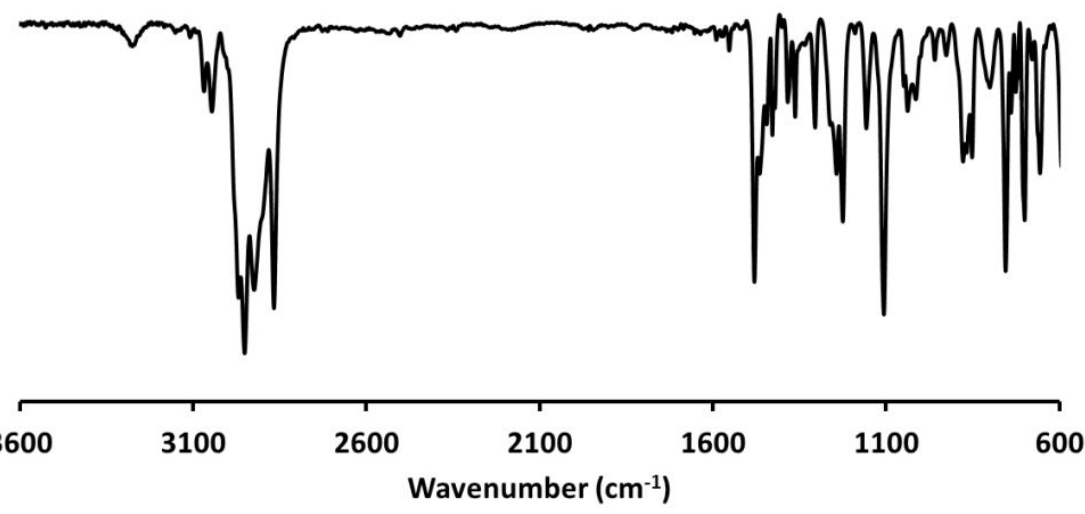

Figure S64. IR spectrum of $\mathbf{4 b}$ at room temperature. (KBr pellet)

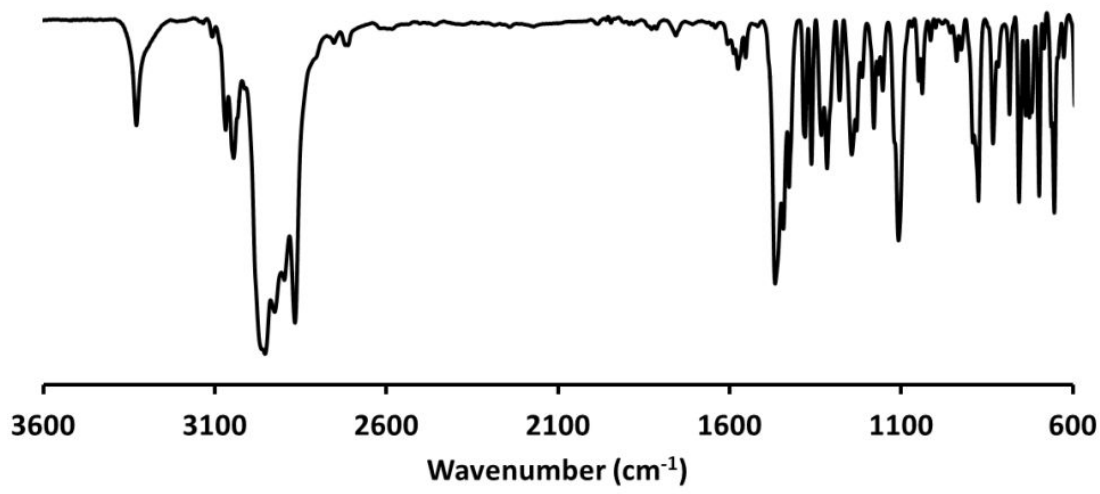


Figure S65. IR spectrum of 5 at room temperature. (KBr pellet)

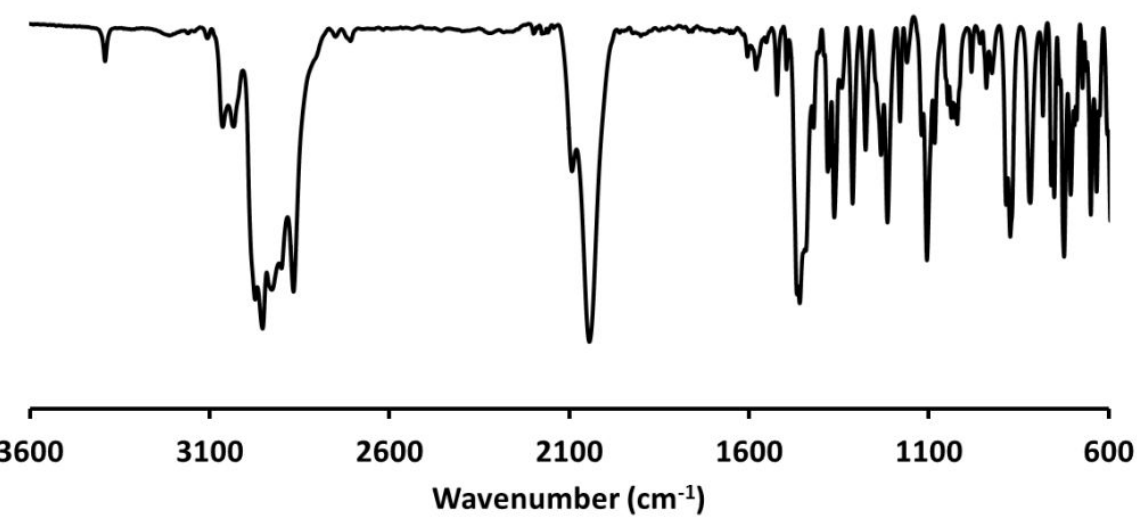

Figure S66. IR spectrum of $\mathbf{6 a}$ at room temperature. (KBr pellet)
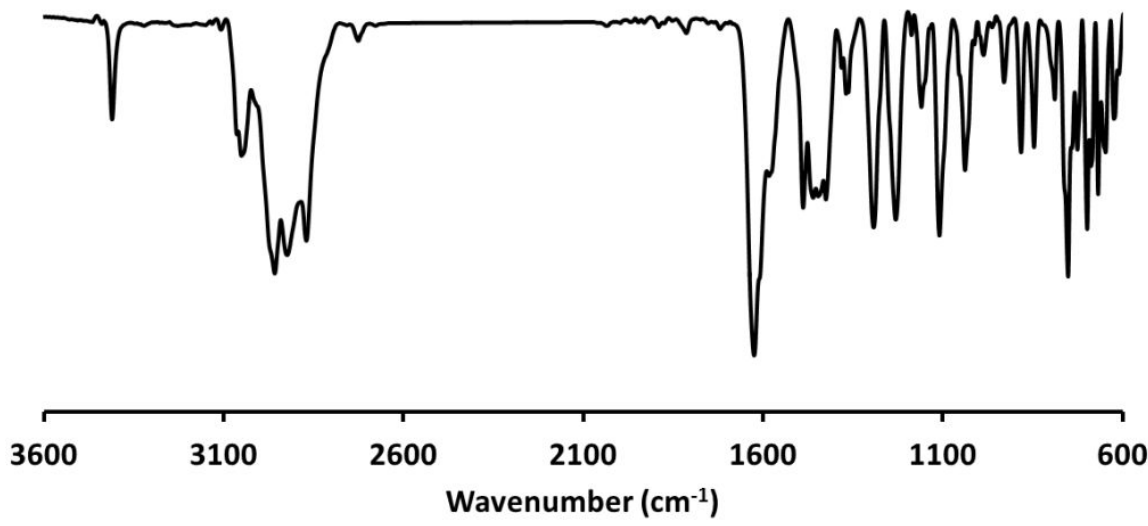

Figure S67. IR spectrum of $\mathbf{6 b}$ at room temperature. (KBr pellet)

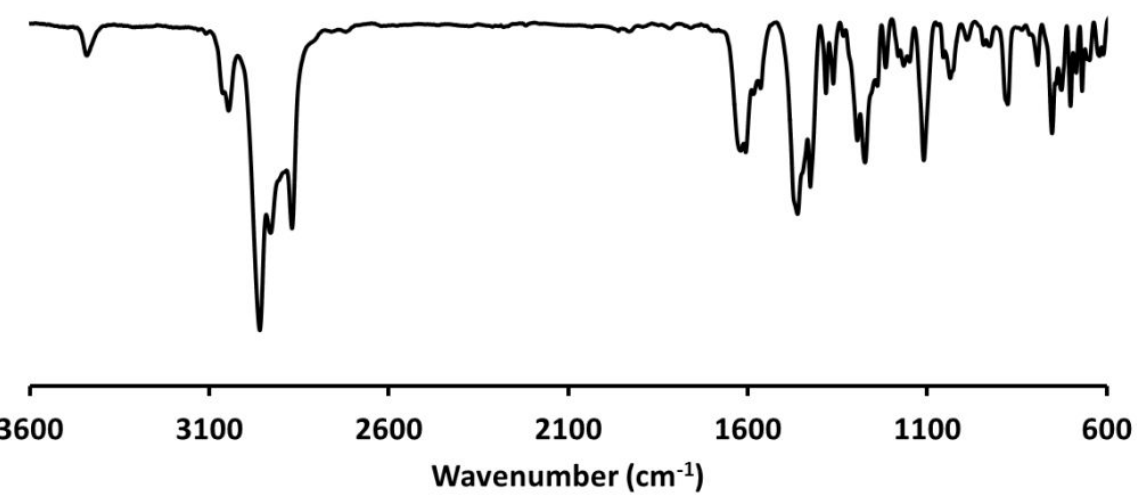


Computational details. Single point calculations, geometry optimizations and frequency calculations were performed with the Gaussian $09^{3}$ with the B3LYP functional ${ }^{4-6}$ theory. The $6-311+\mathrm{g}^{* *}$ basis set was used for all other atoms. The initial geometries for computational models of $\mathbf{2} \mathbf{a}, \mathbf{3} \mathbf{b}$ and were obtained from its corresponding X-ray crystal structure. The initial geometries for computational models of 2b, 3a, IS1-1, IS1-2, IS2-1 and IS2-2 were obtained from X-ray crystal structure of those analogous compound. Optimization was conducted with default solvent of benzene. Optimized structures were verified using frequency calculations to confirm the absence of any imaginary frequency.

Figure S68. Optimized structure of 2a. All hydrogen atoms except the $\mathrm{N}-\mathrm{H}$ and $\mathrm{C} 37-\mathrm{H}$ are omitted for clarity.

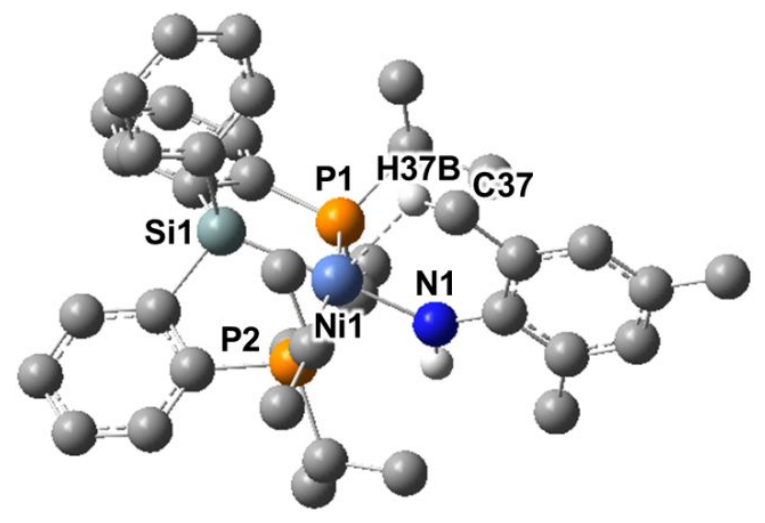

Table S9. Selected bond distances and angles for $\mathbf{2 a}\left(\AA\right.$ and $\left.^{\circ}\right)$.

\begin{tabular}{cccccc}
\hline Distance & XRD structure & DFT & Angle & XRD structure & DFT \\
\hline $\mathrm{d}_{\mathrm{Ni1}-\mathrm{P} 1}$ & $2.1935(6)$ & 2.2498 & $\angle \mathrm{P} 1-\mathrm{Ni1}-\mathrm{P} 2$ & $153.41(2)$ & 155.61 \\
\hline $\mathrm{d}_{\mathrm{Ni1}-\mathrm{P} 2}$ & $2.2083(6)$ & 2.2798 & $\angle \mathrm{P} 1-\mathrm{Ni1}-\mathrm{N} 1$ & $93.75(6)$ & 92.60 \\
& & & $\angle \mathrm{P} 2-\mathrm{Ni1}-\mathrm{N} 1$ & $98.29(6)$ & 97.75 \\
\hline $\mathrm{d}_{\mathrm{Ni1}-\mathrm{N} 1}$ & $1.960(2)$ & 1.9766 & $\angle \mathrm{Si1}-\mathrm{Ni1}-\mathrm{N} 1$ & $177.17(6)$ & 177.07 \\
\hline $\mathrm{d}_{\mathrm{Ni1}-\mathrm{Si} 1}$ & $2.2204(6)$ & 2.2765 & $\angle \mathrm{Si1}-\mathrm{Ni1}-\mathrm{P} 1$ & $84.69(2)$ & 84.94 \\
\hline $\mathrm{d}_{\mathrm{Ni1}-\mathrm{C} 37}$ & $2.962(2)$ & 3.1081 & $\angle \mathrm{Ni1}-\mathrm{H} 37 \mathrm{~B}-$ & $84.10(2)$ & 83.94 \\
$\mathrm{~d}_{\mathrm{Ni1}-\mathrm{H} 37 \mathrm{~B}}$ & 2.0294 & 2.2929 & $\mathrm{C} 37$ & 158.2 & 129.41 \\
\hline
\end{tabular}


Figure S69. Optimized structure of $\mathbf{2 b}$. All hydrogen atoms except the N-H and C37-H are omitted for clarity.

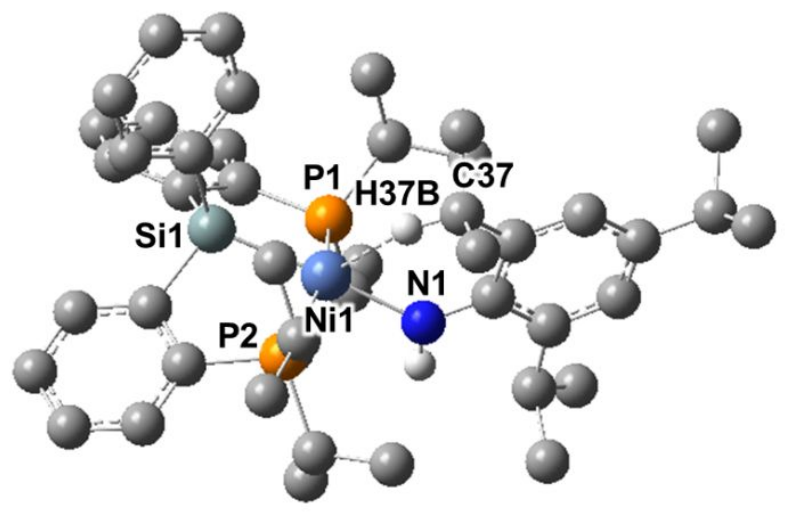

Table S10. Selected bond distances and angles for $\mathbf{2 b}\left(\AA\right.$ and $\left.^{\circ}\right)$.

\begin{tabular}{|c|c|c|c|}
\hline Distance & DFT & Angle & DFT \\
\hline $\mathrm{d}_{\mathrm{Ni1}-\mathrm{P} 1}$ & 2.2764 & $\angle \mathrm{P} 1-\mathrm{Ni1}-\mathrm{P} 2$ & 154.48 \\
\hline \multirow{2}{*}{$\mathrm{d}_{\mathrm{Ni1}-\mathrm{P} 2}$} & \multirow{2}{*}{2.2845} & $\angle \mathrm{P} 1-\mathrm{Ni} 1-\mathrm{N} 1$ & 97.11 \\
\hline & & $\angle \mathrm{P} 2-\mathrm{Ni1}-\mathrm{N} 1$ & 94.23 \\
\hline $\mathrm{d}_{\mathrm{Ni} 1-\mathrm{N} 1}$ & 1.9981 & $\angle \mathrm{Si} 1-\mathrm{Ni} 1-\mathrm{N} 1$ & 177.31 \\
\hline \multirow{2}{*}{$\mathrm{d}_{\mathrm{Ni1}-\mathrm{Si} 1}$} & \multirow{2}{*}{2.2897} & $\angle \mathrm{Si} 1-\mathrm{Ni} 1-\mathrm{P} 1$ & 84.63 \\
\hline & & $\angle \mathrm{Si} 1-\mathrm{Ni} 1-\mathrm{P} 2$ & 83.37 \\
\hline $\mathrm{d}_{\mathrm{Ni1}-\mathrm{C} 37}$ & 3.3791 & \multirow{2}{*}{$\angle \mathrm{Ni1}-\mathrm{H} 37 \mathrm{~B}-\mathrm{C} 37$} & \multirow{2}{*}{151.30} \\
\hline $\mathrm{d}_{\mathrm{Ni1}-\mathrm{H} 37 \mathrm{~B}}$ & 2.3761 & & \\
\hline
\end{tabular}


Table S11. Cartesian coordinates of the optimized geometries.

$==$

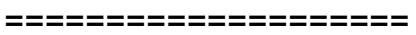

$\mathrm{Ni0} .302940 .015503-0.215479$

$\begin{array}{lll}\text { N } 2.234571 & 0.013651 & -0.63449\end{array}$

H $2.439362 \quad 0.213726-1.603532$

$\begin{array}{llll}P & 0.130787 & 2.235357 & -0.538082\end{array}$

$P-0.108456-2.184082-0.651389$

Si-1.935221 0.104570 .190774

H 1.306020 .1212331 .843668

C $3.393838-0.1155210 .080709$

C -1.617768 $2.840979-0.463994$

C $0.6913412 .608075 \quad-2.30366$

C 1.063223 .4687310 .536036

C -1.872269 -2.335366 -1.220584

C $0.130086-3.4899310 .696678$

C $0.85725-2.906947-2.100992$

C -2.58355-0.190241.971991

C -2.72063-1.25327-0.903079

C -2.591154 $1.856501 \quad-0.215007$

C $2.112154-0.4978312 .259053$

C $3.399878-0.3414941 .489685$

$\begin{array}{lll}\text { C } 4.673668 & -0.004047 & -0.560174\end{array}$

C -1.993446 4.18349 -0.626052

C -0.194472 $1.856502 \quad-3.308757$

H $1.6948862 .173051-2.329036$

$\begin{array}{lll}\text { C } 0.797629 & 4.089908 & -2.690105\end{array}$

C $0.631923 \quad 3.357027 \quad 2.005435$

H $0.749747 \quad 4.452146 \quad 0.169579$

C $2.588167 \quad 3.361983 \quad 0.398267$

C -2.359148 $-3.414153-1.972047$

C -0.685306 -3.163572 1.953035

H $1.192948-3.3865240 .940744$

C -0.130659 -4.9396130 .260919$

C $0.766271 \quad-2.005418-3.339019$

H $0.352355-3.847625 \quad-2.339929$

C $2.315914-3.226578-1.742755$

C -1.981875 $0.459815 \quad 3.06338$

C -3.691255 -1.009896 2.244054

C -4.04132-1.28034-1.376738

C -3.937788 $2.250464 \quad-0.147189$

H $1.750674 \quad-1.5309712 .248014$

H $2.24227-0.2150583 .307569$

C $4.609358-0.4424382 .180291$

C $5.847374-0.1226580 .178261$

C $4.751104 \quad 0.266084 \quad-2.042659$

$\mathrm{H}-1.2487584 .95336-0.787096$

C -3.335074.548356 -0.569059

H $0.217744 \quad 1.961393 \quad-4.317213$

$\mathrm{H}-1.2090112 .263803 \quad-3.319699$

H $-0.2622580 .792145 \quad-3.080034$

H $1.2369894 .166212 \quad-3.690175$

H $1.4306784 .665833-2.013113$

H -0.185514 $4.563665-2.733315$

H - 0.4491873 .4520512 .128375

H 1.1045894 .1552352 .586107
$\begin{array}{lll}\mathrm{H} 0.949617 & 2.405234 & 2.43788\end{array}$

H 3.0575874 .1460551 .001378

H $2.925761 \quad 3.493657 \quad-0.631536$

H $2.958948 \quad 2.398171 \quad 0.748662$

$\mathrm{H}-1.716834-4.24865-2.225396$

C -3.675688 -3.42108-2.426814

H -0.424376 -3.8672592 .749802$

H -1.757195 -3.2588561 .765201$

H $-0.500467-2.1548532 .320996$

H $0.127188-5.611247 \quad 1.08617$

H 0.462006 -5.24558-0.602715

$\mathrm{H}-1.186636 \quad-5.1006630 .030121$

H $1.241452-2.502967-4.190504$

H $1.284516-1.059423-3.174824$

H -0.268076 -1.788597 -3.615733

H 2.829176 -3.60985-2.630792

H $2.396848-3.989702-0.966437$

H $2.840331-2.335699-1.398594$

$\mathrm{H}-1.1198 \quad 1.0979942 .901798$

C -2.461025 $0.303113 \quad 4.362865$

C -4.179842 -1.169205 3.542

$\mathrm{H}-4.182893-1.5408441 .435584$

C -4.51698-2.348163 -2.135192

H -4.710236 -0.453356 -1.163799

C -4.310983 $3.577962 \quad-0.334811$

$\mathrm{H}-4.7063541 .519455 \quad 0.082015$

H $4.569872-0.6041773 .255797$

C $5.855205-0.3447 \quad 1.559571$

H $6.796009-0.028863-0.346757$

H $5.790613 \quad 0.318987 \quad-2.372919$

H $4.2731591 .217668-2.31302$

H $4.257359-0.514241-2.63596$

H -3.618749 $5.587381-0.695931$

$\mathrm{H}-4.039248$-4.25623-3.015416

$\mathrm{H}-1.9724490 .816111 \quad 5.184743$

C -3.564971 -0.513935 4.607079

$\mathrm{H}-5.03822-1.8086983 .720447$

H -5.53864-2.343796 -2.49981

H $-5.356333 \quad 3.862422 \quad-0.279266$

C $7.146336-0.494482 .330345$

H -3.939846 -0.639263 5.617062

H $6.990075-0.3276143 .399809$

H $7.9043190 .219262 \quad 1.991726$

H 7.578679 -1.497068 2.219553

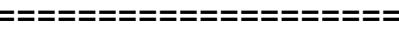

$2 \mathrm{~b}$

$\mathrm{Ni}-0.3153-0.136842-0.270922$

N $1.536318-0.686109-0.782922$

H $1.596863-0.692425-1.791958$

P -0.069485 $2.090505-0.67155$

P -1.209874 $-2.208879-0.625052$

Si-2.475162 $0.414628 \quad 0.252003$

H $0.947244 \quad-0.9101911 .587463$

C $2.792531-0.510617-0.246729$

C -1.692589 $2.987643 \quad-0.631306$ 


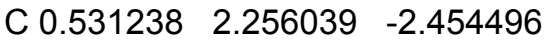
$\begin{array}{llll}C & 1.026313 \quad 3.271886 & 0.307644\end{array}$ C $-3.000348-2.017013-1.082527$ C - $1.157584-3.4798180 .772189$ C $-0.515351-3.187861-2.082029$ C -3.070360.331935 2.073983 C $-3.592284-0.785607-0.734405$ C - $2.8102612 .229245 \quad-0.245042$ C $1.900075 \quad-1.0495942 .111773$ C $3.01677-0.6168251 .166357$ C $3.949893-0.261626-1.065552$ C $-1.832164 .354688-0.919631$ C $-0.482461 \quad 1.614951 \quad-3.414222$ H $1.4363111 .64293-2.444266$ $\begin{array}{lll}\text { C } 0.907132 & 3.662599 & -2.943019\end{array}$ C $0.553062 \quad 3.385765 \quad 1.762665$ H $0.862651 \quad 4.242766 \quad-0.171032$ C $2.520782 .942683 \quad 0.230273$ C $-3.744923-2.988031-1.767817$ C - $1.723143-2.9181832 .08091$ H -0.083597 -3.6348380 .906929$ C -1.812104 -4.8300730 .445062$ C $-0.470816-2.343214-3.362256$ H - $1.239833-3.990146-2.248585$ C $0.844032-3.836076-1.779275$ C $-2.320197 \quad 0.934042 \quad 3.099052$ C $-4.280762-0.280062 .43956$ C -4.926528 $-0.558809-1.107199$ C $-4.0570992 .871145 \quad-0.155897$ $\begin{array}{lll}\text { C } 4.294947 & -0.437715 & 1.69161\end{array}$ C $5.202459-0.101443-0.472912$ C $3.848632-0.215645-2.589829$ H $-0.9737064 .957649 \quad-1.188007$ C $-3.080574 .964523 \quad-0.84788$ $\mathrm{H}-0.0619091 .570702 \quad-4.423823$ $\mathrm{H}-1.4029142 .203173 \quad-3.462538$ H $-0.7490330 .600605 \quad-3.114621$ H $1.375133 \quad 3.579864 \quad-3.929521$ H $1.618193 \quad 4.170576 \quad-2.289821$ H $0.026782 \quad 4.298394 \quad-3.059769$ H -0.495253 $3.682307 \quad 1.835045$ H $1.151166 \quad 4.139954 \quad 2.283528$ H $0.681656 \quad 2.440787 \quad 2.294427$ H $3.085733 .722228 \quad 0.751843$ H $2.890396 \quad 2.900677 \quad-0.795763$ H $2.752669 \quad 1.987176 \quad 0.701233$ $\mathrm{H}-3.30253-3.93752-2.043083$ C $-5.067948-2.740744-2.124937$ $\mathrm{H}-1.555284 \quad-3.6376532 .888539$ $\mathrm{H}-2.79944-2.7471172 .010376$ $\mathrm{H}-1.254374-1.9761362 .366199$ H $-1.645713-5.519807 \quad 1.27887$ H - $1.403908-5.300853-0.45095$ $\mathrm{H}-2.892502-4.726920 .318008$ H -0.206093 -2.98013-4.212072 H $0.280583-1.554748-3.29687$ H -1.434968 $-1.879368-3.582575$
H $1.214689-4.335108-2.680616$ H $0.775309-4.593089-0.99566$ H $1.573154-3.087841-1.467474$ $\mathrm{H}-1.377271 \quad 1.416432 \quad 2.866074$ C -2.755258 0.928834 .423337 C $-4.725671-0.2861383 .762598$ $\mathrm{H}-4.889116-0.7661071 .68436$ C -5.659069 $-1.520704-1.799064$ H $-5.4034860 .384901 \quad-0.867157$ C -4.198012 $4.220055-0.46604$ $\mathrm{H}-4.9278642 .320495 \quad 0.184147$ H $4.423747 \quad-0.5317612 .765937$ C $5.415888-0.166580 .904037$ H $6.0601390 .090336-1.111176$ H $2.827511 \quad 0.079338-2.857441$ H -3.180039 $6.020159-1.075785$ H -5.631829 $-3.494852-2.663006$ $\mathrm{H}-2.1517661 .400003 \quad 5.192123$ C -3.963124 0.317774 .760433 H -5.66624-0.765848 4.013247 H -6.685711 $-1.320037-2.086252$ H $-5.1696814 .696916-0.393996$ C $6.80266 \quad 0.029258 \quad 1.498728$ $\mathrm{H}-4.304275 \quad 0.311266 \quad 5.78994$ H $7.475524 \quad 0.253126 \quad 0.661616$ $\begin{array}{lll}\text { C } 6.864457 & 1.226033 & 2.466546\end{array}$ H 6.2164091 .0651313 .333868 H 7.883821 .3788742 .837012 H $6.5383182 .146255 \quad 1.974384$ C $7.334075 \quad-1.246282 \quad 2.180335$ H $7.335878-2.0902331 .485381$ H $8.356795 \quad-1.1000242 .544387$ H $6.712724-1.5236023 .037907$ $\begin{array}{lll}\text { C } 4.76474 & 0.828066 & -3.254149\end{array}$ H $4.631751 .818112-2.810019$ H $5.8214090 .561573-3.165661$ H $4.539778 \quad 0.899803 \quad-4.323053$ $\begin{array}{llll}\text { C } 4.094795 & -1.605834 & -3.213241\end{array}$ H $3.420837-2.356918-2.795731$ H $3.951714-1.583941-4.2994$ H $5.119767-1.933161-3.013176$ C $1.827092 \quad-0.2401493 .418143$ H $0.937128-0.5183193 .991511$ H $2.694875 \quad-0.4255644 .057864$ H $1.7855 \quad 0.833975 \quad 3.225515$ C $2.038989-2.5506712 .439726$ H $2.966472-2.7319162 .991926$ H $1.206431-2.9041653 .056998$ H $2.078467 \quad-3.1520481 .52841$

=ニニニニニニニニニニニニニニニニニニ

3a

ニニニニニニニニニニニニニニニニニニニニ

Ni-0.878962 $-0.527984-0.045079$ $\begin{array}{llll}\text { P }-2.494368 & 0.949161 & -0.460337\end{array}$ P $0.684625 \quad-1.576938 \quad 1.171928$ Si $0.44722 \quad 1.319226 \quad 0.344549$ C -1.971547 $-2.096364-0.596709$ 
H - $2.524091 \quad-0.0641142 .325421$

C $-1.962868 \quad 2.709358 \quad-0.170359$

C $-3.356646 \quad 1.065978-2.132628$

C $-3.8900240 .672485 \quad 0.779516$

C $1.294585-0.3469962 .441322$

C $2.285393-2.081310 .297147$

C $0.213352-3.0562 .245668$

N $1.747504 \quad 1.892071 \quad-0.763871$

C -0.641609 $2.888508 \quad 0.276155$

C $1.180971 \quad 1.011252 .081317$

C $-1.634876-2.671121-1.842087$

C $-3.059703-2.7015770 .061584$

C $-3.3319510 .661132 \quad 2.210367$

C $-2.7923313 .822448 \quad-0.378047$

$\begin{array}{llll}C-2.319807 & 1.254435 & -3.248814\end{array}$

$\mathrm{H}-3.961413 \quad 1.976917 \quad-2.078057$

C $-4.291781-0.112187-2.439553$

C $-5.0985811 .613363 \quad 0.677839$

$\mathrm{H}-4.221624-0.339770 .53414$

C $1.823196 \quad-0.6971283 .691987$

C $2.033784-2.78147-1.046501$

H $2.733924-1.1097 \quad 0.070923$

C $3.279624 \quad-2.8589451 .169944$

C $-0.073287-4.3355651 .445235$

H $1.092665 \quad-3.2477972 .868475$

C - $0.961119-2.710393 .173289$

H $1.3050962 .468397 \quad-1.472905$

C $2.890152 \quad 1.243609-1.317929$

C -0.185896 $4.195451 \quad 0.518579$

C $1.571264 \quad 1.988086 \quad 3.009035$

$\mathrm{H}-0.808279-2.261759-2.418428$

C $-2.319598-3.760188-2.386676$

C $-3.758277-3.791752-0.467175$

$\mathrm{H}-3.385087-2.3248031 .027792$

$\mathrm{H}-2.9454541 .644144 \quad 2.494058$

H -4.1263170 .4000372 .917082$

H $-3.8018753 .701554-0.750292$

C -2.324305 $5.10792-0.12065$

$\mathrm{H}-1.702306 \quad 0.360502 \quad-3.362793$

H -1.661437 $2.105425 \quad-3.059142$

$\mathrm{H}-2.828498 \quad 1.432237 \quad-4.201501$

H $-4.7802680 .06278-3.404062$

H $-5.077877-0.229571-1.691521$

H -3.745408 - $1.053395-2.502971$

H -5.890939 $1.248117 \quad 1.339671$

H $-5.5169851 .665522 \quad-0.329505$

$\mathrm{H}-4.8479632 .625864 \quad 1.000889$

H $1.928012 \quad-1.7353043 .98294$

C $2.206702 \quad 0.290051 \quad 4.597109$

H $2.990567-2.948706-1.550413$

H $1.415188-2.170441-1.704826$

H $1.543581-3.749561-0.929356$

H $2.921389-3.863631 .408156$

H $3.500832-2.3410492 .105627$

H $4.223753-2.969780 .627186$

H $-0.365347 \quad-5.1310442 .138972$

H $0.802947 \quad-4.6872820 .899064$
H -0.88766-4.193711 0.734962

H - $1.875138-2.5493492 .601193$

H $-0.769807-1.8189923 .773845$

H -1.141904 -3.543436 3.8606

$\begin{array}{llll}C & 4.111145 & 1.198387 & -0.61384\end{array}$

$\begin{array}{lll}\text { C } 2.832605 & 0.709281 & -2.627609\end{array}$

C -1.018399 $5.295938 \quad 0.334534$

$\mathrm{H} 0.839417 \quad 4.3579 \quad 0.833696$

C $2.069884 \quad 1.636978 \quad 4.260681$

H $1.479642 \quad 3.039227 \quad 2.756824$

H -2.015693 $-4.163812-3.348623$

C -3.39145-4.331323 -1.699083

$\mathrm{H}-4.589519-4.2202610 .086091$

H -2.973538 $5.961438 \quad-0.283461$

H $2.601719 \quad 0.007217 \quad 5.566883$

$\begin{array}{lll}\text { C } 5.217408 & 0.577651 & -1.207137\end{array}$

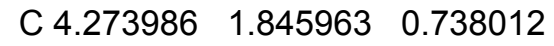

$\begin{array}{lll}\text { C } 3.965894 & 0.117902 & -3.184899\end{array}$

$\begin{array}{lll}\text { C } 1.556382 & 0.783132 & -3.427566\end{array}$

H -0.649632 $6.297571 \quad 0.528649$

H $2.3523222 .406223 \quad 4.971603$

H -3.927591 $-5.178022-2.114555$

$\begin{array}{lll}\text { H } 6.153068 & 0.554017 & -0.655191\end{array}$

$\begin{array}{lll}\text { C } 5.171668 & 0.025042 & -2.485576\end{array}$

H $3.8974681 .214463 \quad 1.546346$

H $3.901572 \quad-0.287195-4.191171$

H $0.716396 \quad 0.35129-2.876809$

$\begin{array}{lll}\text { C } 6.376633 & -0.650942 & -3.09615\end{array}$

H $6.521949-0.346159-4.136436$

H $7.288259-0.410765-2.544321$

H $6.266403-1.741101-3.091637$

H $1.2795821 .817658 \quad-3.664477$

H $1.659287 \quad 0.249374 \quad-4.374145$

H 3.7180462 .7841590 .7869

H $5.328596 \quad 2.048497 \quad 0.938366$

ニニニニニニニニニニニニニニニニニニニ

3b

$\mathrm{Ni}-1.461893 \quad-0.5368950 .037232$

$\begin{array}{llll}\text { P }-2.921774 & 0.985974 & -0.681933\end{array}$

P $-0.1554 \quad-1.5587241 .543937$

Si-0.077202 $1.263341 \quad 0.453824$

C $-2.569443-2.0844 \quad-0.542242$

H $-3.3448220 .187842 \quad 2.15239$

C -2.315104 $2.732954-0.472502$

$\begin{array}{lll}\text { C }-3.584627 & 1.000045 & -2.445721\end{array}$

$\begin{array}{lll}\text { C }-4.461687 & 0.889842 & 0.405235\end{array}$

C $0.347316-0.256272 .78533$

C $1.517376-2.217436 \quad 0.952176$

C - $0.863229-2.9158882 .648415$

N $1.385471 \quad 1.681835 \quad-0.507234$

C - $1.0515582 .877345 \quad 0.127552$

C 0.396251 .0656212 .297957

C $-2.100039-2.764772-1.687251$

C $-3.770001-2.586218-0.003985$

C $-4.0705320 .959097 \quad 1.888457$

C -3.037058 $3.867628 \quad-0.874296$ 
C -2.416642 $1.038011 \quad-3.440538$ $\mathrm{H}-4.1377361 .940418 \quad-2.536042$ C $-4.544932-0.154132-2.764094$ C $-5.5850091 .889305 \quad 0.094797$ H $-4.831617-0.1171950 .19541$ C $0.64468-0.5188454 .130357$ C $1.405188-3.055206-0.329712$ H $2.042796-1.2946850 .687793$ C $2.344795 \quad-2.9333212 .02833$ C -1.107773 -4.2453991 .91829$ H -0.095949 -3.0962613 .407915$ C $-2.136521-2.4357443 .360794$ H $1.1005342 .279365-1.27459$ $\begin{array}{llll}C & 2.554734 & 0.934815 & -0.859362\end{array}$ C $-0.547224 .173893 \quad 0.327048$ C $0.719774 \quad 2.097671 \quad 3.191589$ $\mathrm{H}-1.181191-2.438481-2.169199$ C $-2.766266-3.856343-2.249498$ C $-4.453204-3.676001-0.55332$ H $-4.201001-2.1247950 .880639$ H -3.637841 $1.931188 \quad 2.141497$ H $-4.958145 \quad 0.817753 \quad 2.513659$ H -3.997343.771241 -1.365421 C $-2.522143 \quad 5.143128-0.660007$ $\mathrm{H}-1.837125 \quad 0.112861 \quad-3.393563$ $\mathrm{H}-1.7431741 .876813-3.24991$ H - 2.799691.142123 -4.460572 H -4.908951 -0.04129-3.790882 H $-5.417115-0.168253-2.107946$ H -4.048841 $-1.121521-2.685324$ H $-6.470377 \quad 1.624141 \quad 0.682173$ H $-5.8810571 .887237 \quad-0.956389$ H $-5.3048612 .907618 \quad 0.372558$ H $0.619398 \quad-1.5290974 .520363$ C $0.959204 \quad 0.5227594 .999952$ H $2.40907-3.290229-0.695888$ H $0.882162-2.512247-1.117113$ H $0.878777-3.997217-0.167748$ H $1.897074 \quad-3.8844192 .327813$ H 2.47974 -2.320854 2.922225 H $3.340368-3.1529591 .629914$ $\mathrm{H}-1.56333-4.9556822 .6163$ H $-0.181886-4.6948741 .557357$ $\mathrm{H}-1.784996-4.1250651 .072861$ H -2.941908 -2.266784 2.645774 H - 1.97732-1.513695 3.923128 $\mathrm{H}-2.47271-3.2028564 .06605$ C $3.690363 \quad 0.947118-0.014487$ C $2.621150 .238368 \quad-2.093394$ C $-1.276081 \quad 5.298216 \quad-0.050963$ H $0.437115 \quad 4.307775 \quad 0.763421$ C $0.988365 \quad 1.836427 \quad 4.532655$ H $0.749609 \quad 3.124595 \quad 2.842351$ H $-2.357738-4.344028-3.13031$ C $-3.954125-4.321702-1.683543$ H $-5.375786-4.021499-0.094893$ H $-3.0878296 .013544 \quad-0.974443$
H 1.1737210 .308966 .041485

$\begin{array}{llll}\text { C } 4.822074 & 0.213238 & -0.383138\end{array}$

C $3.762583 \quad 1.833674 \quad 1.224165$

$\begin{array}{llll}\text { C } 3.785629 & -0.461304 & -2.419458\end{array}$

$\begin{array}{lll}\text { C } 1.467211 & 0.308058 & -3.087777\end{array}$

H -0.8710156 .291380 .112062$

H 1.2180372 .650585 .21196

H -4.477301 $-5.169304-2.11345$

H $5.685253 \quad 0.232065 \quad 0.274192$

$\begin{array}{llll}\text { C } 4.894161 & -0.507257 & -1.574508\end{array}$

$\begin{array}{llll}\text { C } 4.620591 & 3.081881 & 0.933766\end{array}$

H $2.7533112 .179908 \quad 1.43269$

C 4.2721691 .102332 .476532

H $3.834116 \quad-0.997259-3.361041$

$\begin{array}{lll}\text { C } 1.581147 & 1.576358 & -3.962015\end{array}$

H $0.5466890 .389125-2.50288$

C $1.326664-0.928769-3.98878$

C $6.138643 \quad-1.291171-1.967892$

H 5.6572682 .8081880 .713776

H $4.626505 \quad 3.7544851 .79785$

H $4.231706 \quad 3.635277 \quad 0.074746$

$\begin{array}{lll}\text { H } 5.308346 & 0.769162 & 2.364423\end{array}$

$\begin{array}{lll}\text { H } 3.658632 & 0.227679 & 2.704718\end{array}$

H $4.235671 \quad 1.769558 \quad 3.342508$

H $1.6463892 .489898 \quad-3.364098$

H $0.7163251 .670851-4.625716$

H $2.481526 \quad 1.529869-4.582354$

H $2.150974-1.011831-4.702932$

H $0.403369-0.861088-4.57109$

H $1.291681-1.852935-3.407201$

$\begin{array}{lll}\text { C } 7.352263 & -0.370447 & -2.193998\end{array}$

H $5.916069-1.778302-2.924401$

C $6.473516-2.400415-0.954412$

H 7.130203 $0.399255 \quad-2.937778$

H $8.214068 \quad-0.946308-2.545672$ H $7.6444 \quad 0.133965 \quad-1.26801$

H $6.720773 \quad-1.9808730 .025412$

H $7.334433-2.985564-1.292411$

$\begin{array}{llll}\text { H } 5.629783 & -3.083007 & -0.822615\end{array}$

==ニ==ニ====ニ=ニ=-===ニ=

IS1-1

=ニニ=ニ=ニ=ニ=ニ=ニ=ニ=ニ==

P 2.608513 -1.17733-1.023722

P $1.079535 \quad 2.560154 \quad-0.044703$

Si-0.565251 -0.6136980 .789929$

C -2.425979 $0.181196-1.337615$

C $-2.613454-0.401535-2.618398$

C $1.629476-2.519619-0.175704$

C $3.218783-1.969247-2.627064$

C $4.200572-1.100060 .032182$

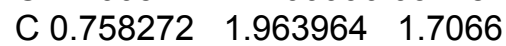

C $-0.466873 .568447 \quad-0.469138$

C 2.462113 .840060 .150709

C $0.480756-2.2513950 .622636$

$\begin{array}{lll}\text { C } 0.293836 & 0.641202 & 1.965326\end{array}$

C $-3.489030 .906156-0.76245$

C -3.831349 $-0.260318-3.277346$ 
C $2.12582-3.830842-0.255008$ C $2.051627-2.358368-3.541486$ H $3.77919-2.873993-2.3773$

C $4.171022-0.999943-3.346005$ C $5.161261-2.29528-0.033713$

H $4.710033-0.230297-0.39722$ C $3.860295 \quad-0.773591 .492792$ C $1.166008 \quad 2.760102 \quad 2.786896$ C -0.629928 $3.788222-1.98142$ $\mathrm{H}-1.2553512 .878988 \quad-0.167395$ C $-0.640394 .867803 \quad 0.326287$ C $2.747708 \quad 4.525155 \quad-1.196378$ H $2.137954 \quad 4.6106120 .856756$ C $3.747423 .186415 \quad 0.680303$ C -2.189568 -1.197591 1.636151 C - $0.024415-3.332751 .375596$ C $0.392594 \quad 0.169229 \quad 3.286984$ C $-4.7023 \quad 1.004046-1.459408$ $\mathrm{H}-3.948874-0.716435-4.256768$ C -4.903842 $0.43623-2.71168$ H $2.973356-4.045091-0.892842$ C $1.573267-4.880770 .471333$ H $1.474571-1.477505-3.833688$ H $1.370738-3.065227-3.062773$ H $2.433016-2.826148-4.455289$ H $4.526105-1.449762-4.279163$ H $5.048956-0.750701-2.745384$ H $3.660203-0.065312-3.595904$ H $6.063436-2.0615840 .542246$ H $5.48061-2.533488-1.050356$ H $4.720836-3.1919740 .408576$ H $3.354805-1.6142811 .975889$ H $3.212798 \quad 0.099657 \quad 1.576239$ H $4.780309-0.5733752 .052245$ H $1.494283 \quad 3.777018 \quad 2.613149$ C 1.205912 .2762894 .093963 $\mathrm{H}-1.6283494 .186924 \quad-2.189069$ $\mathrm{H}-0.5229652 .852036 \quad-2.533513$ H $0.095949 \quad 4.499064 \quad-2.381964$ H $0.108178 \quad 5.617366 \quad 0.054671$ H -0.578263 $4.699103 \quad 1.404052$ $\mathrm{H}-1.6238965 .302914 \quad 0.118206$ H $3.615915 \quad 5.184517 \quad-1.094313$ H $1.9129715 .135378-1.542388$ H $2.9767373 .78972-1.97365$ H $4.0982942 .417409-0.013713$ H $3.610066 \quad 2.718541 \quad 1.655947$ H 4.5344343 .9421920 .77686 C -3.007319 -2.088785 0.915108 C - $2.65333-0.7928632 .897062$ C $0.505596-4.6195031 .318689$ H $-0.876873-3.1763722 .021803$ $\begin{array}{llll}\text { C } 0.852276 & 0.956733 & 4.341304\end{array}$ H $0.103177 \quad-0.8531833 .507489$ H $-5.511943 \quad 1.562906 \quad-0.997166$ H $1.984939-5.8804310 .383687$ H $1.537446 \quad 2.921874 \quad 4.900035$
C -4.214265 -2.559167 1.426741 C -3.858795 -1.2634083 .421769$ H $0.069992-5.4090061 .921575$ H $0.919454 \quad 0.542898 \quad 5.341562$ H -4.819321 -3.2415010 .839487$ C -4.643781 -2.1501372 .689088$ $\mathrm{H}-4.185015-0.9294534 .401191$ $\mathrm{H}-2.081083-0.0869013 .485881$ H $-5.581915-2.5148543 .093309$ H - $2.699363-2.427011-0.068616$ C -6.219883 $0.566265 \quad-3.441275$ H -6.666201 $-0.414102-3.63802$ H -6.938683 $1.148301-2.860326$ H $-6.093111 .062039-4.409234$ C $-1.499613-1.176415-3.274208$ H $-0.647682-0.533804-3.522866$ $\mathrm{H}-1.116617-1.961601-2.616473$ $\mathrm{H}-1.843168$-1.638699 -4.201568 C -3.413996 $1.609076 \quad 0.573255$ H -3.773814 2.6383290 .47835 H -4.0445051 .1099891 .313272$ H -2.406358 $1.639567 \quad 0.9803$ $\mathrm{N}-1.1466520 .052006-0.724686$ H $-0.3828950 .157676-1.396686$ Ni1.717721 $0.759235 \quad-1.048023$ $===================$

IS1-2

$====================$

$\begin{array}{lll}\text { P } 1.444457 & -1.659439 & -1.557226\end{array}$

P $1.068365-0.6177462 .170456$

Si-1.791522 $-0.674041-0.072824$

C - $1.587237 \quad 2.217566 \quad-0.204544$

C - $1.2382132 .925411 \quad-1.382367$

C $-0.230736-1.914854-2.372112$

C $2.58678-1.29551-3.032465$

C $1.942642-3.467868-1.163534$

C $-0.361087-1.823252 \quad 2.353725$

$\begin{array}{lll}\text { C } 0.418133 & 0.804164 & 3.25765\end{array}$

C $2.448167-1.3946423 .21178$

C -1.469278 -1.62903-1.728519

C -1.402924 -1.8610811 .387583$

C -2.416949 $2.845642 \quad 0.743807$

C -1.707567 $4.222292 \quad-1.578467$

C $-0.249788-2.511872-3.643674$

C $2.234640 .030071-3.716901$

H $2.47393-2.097326-3.765915$

C $4.053433-1.288213-2.572116$

C $2.304504-4.385552-2.340966$

H $2.84402-3.325994-0.557195$

$\begin{array}{llll}C & 0.887529 & -4.156362 & -0.289458\end{array}$

C - $0.34769-2.7903113 .370353$

C 1.120812 .1420312 .993574

H $-0.5988880 .902515 \quad 2.87786$

C $0.328145 \quad 0.497054 .758356$

C $3.61857-0.412783 .389158$

H $2.049002-1.6152444 .20572$

C $2.961703 \quad-2.6995642 .585242$ 
C -3.6672 $-0.285483-0.033341$

C $-2.642535-2.060756-2.381908$

C $-2.318437-2.9273561 .459411$

C $-2.879894 .145275 \quad 0.49232$

$\mathrm{H}-1.4275994 .744731 \quad-2.489438$

C -2.539033 $4.85788-0.651262$

H $0.679366-2.701059-4.162884$

C $-1.430038-2.888859-4.277217$

H $2.3642010 .867833-3.028996$

H $1.2059790 .044815 \quad-4.083418$

H $2.897405 \quad 0.191716 \quad-4.574207$

H $4.706499-1.077708-3.426177$

H $4.36697-2.245445-2.148601$

H $4.220389-0.515246-1.818913$

H $2.635231-5.353287-1.947423$

H $3.113129-3.99756-2.96167$

H $1.440219-4.579712-2.980559$

H $-0.019616-4.364818-0.862941$

H $0.6063 \quad-3.554604 \quad 0.57181$

H $1.276214-5.1130280 .075525$

H $0.424269-2.7696314 .128486$

C -1.285905 -3.8189553 .430542$

H 0.5873282 .946123 .51241

H 1.1402632 .376151 .929212

H 2.151664 2.146414 3.354865

H $1.315759 \quad 0.421269 \quad 5.222093$

$\mathrm{H}-0.216058-0.4284964 .959148$

H $-0.2050031 .306972 \quad 5.268465$

H $4.427411-0.9064463 .938652$

H $3.336171 \quad 0.477228 \quad 3.952628$

H $4.013353-0.090312 .422573$

H 3.39468 -2.507846 1.599697

H $2.177435-3.4485262 .468546$

H $3.74568-3.1306443 .217277$

C $-4.2172560 .478901-1.080096$

C $-4.534114 \quad-0.6487331 .009169$

C $-2.638422-2.685699-3.625933$

H -3.602461 $-1.892148-1.912718$

C -2.267651 -3.9033542 .452248$

H -3.097535 -3.0117280 .709143$

$\mathrm{H}-3.525078 \quad 4.611015 \quad 1.232881$

$\mathrm{H}-1.395978-3.347776-5.25954$

$\mathrm{H}-1.232203-4.554364 .226243$

C $-5.5610170 .845383-1.095266$

C $-5.883013-0.2896631 .001112$

$\mathrm{H}-3.573955-2.99329-4.080619$

$\mathrm{H}-2.988943-4.7132052 .457315$

H -5.948656 $1.436608 \quad-1.917838$

$\begin{array}{llll}\mathrm{C} & -6.402717 & 0.456768 & -0.053431\end{array}$

H -6.524062 -0.5906211 .823029$

H -4.159972 -1.2155321 .85287$

H -7.450254 $0.738006 \quad-0.06221$

H $-3.589108 \quad 0.798407 \quad-1.904713$

C $-3.031353 \quad 6.266759 \quad-0.884061$

H $-3.468158 \quad 6.377776 \quad-1.881217$

$\mathrm{H}-3.791273 \quad 6.547216-0.151406$

H -2.213901 $6.992053 \quad-0.807844$
C $-0.3698522 .283638-2.433177$

H $0.635951 \quad 2.076422 \quad-2.057579$

$\mathrm{H}-0.780513 \quad 1.324393 \quad-2.760321$

H $-0.2749432 .933506 \quad-3.305544$

C -2.842463 2.206771 2.044411

H $-2.5129242 .814742 \quad 2.893387$

$\mathrm{H}-3.9316812 .131367 \quad 2.099808$

H -2.440579 1.2059892 .168517

N -1.047546 $0.90811-0.000366$

H - $0.0426830 .878433 \quad-0.204929$

$\begin{array}{llll}\mathrm{Ni} 1.781603 & -0.206311 & 0.102006\end{array}$

C $2.832831 .236269-0.180415$

N $3.536522 \quad 2.168937 \quad-0.383672$

$\begin{array}{llll}\text { C } 4.346993 & 3.362545 & -0.360245\end{array}$

$\begin{array}{llll}\text { C } 4.306111 & 4.007209 & -1.757818\end{array}$

C $3.778636 \quad 4.3303330 .694214$

$\begin{array}{lll}\text { C } 5.788269 & 2.956931 & 0.000736\end{array}$

H $4.6947113 .318662 \quad-2.511195$

H $3.2821854 .274491 \quad-2.027671$

H $4.9153824 .914582-1.768206$

H $3.790901 \quad 3.871775 \quad 1.68489$

H $4.3818845 .241545 \quad 0.724365$

H $2.749514 \quad 4.603281 \quad 0.451955$

H $6.428708 \quad 3.8424120 .017096$

H $5.8213432 .486401 \quad 0.985686$

H $6.186672 \quad 2.253638 \quad-0.733678$

==ニ=ニ=ニ=ニ=ニ=ニ=ニ=ニ=ニ=

IS2-1

Ni1.14872 $0.25314-1.09815$

$\begin{array}{llll}\text { P } 2.490735 & -1.391154 & -0.405463\end{array}$

P $1.2166652 .31841-0.275144$

Si-0.724098 -0.4680170 .699258$

C -0.897944 -0.13376-1.143633

C $-0.257685-1.006047-2.087337$

C $1.395709-2.578086 \quad 0.540148$

C $3.383941-2.467406-1.682657$

C $3.893722-1.0927830 .861605$

C $0.722353 \quad 2.060019 \quad 1.504004$

C - $0.076923 .597473-0.841752$

C $2.810919 \quad 3.341627 \quad-0.188149$

$\mathrm{N}-2.381219-0.552506 \quad 1.382764$

C $0.145306-2.1483751 .059131$

C $0.005652 \quad 0.889293 \quad 1.864055$

C -1.851085 $0.802716 \quad-1.65064$

C $-0.441089-0.772171-3.478447$

H $0.087897-1.984484-1.783466$

C $1.845459-3.8777310 .818147$

C $2.440448-3.152235-2.678603$

H $3.919163-3.248998-1.13761$

$\begin{array}{lll}\text { C } 4.416901 & -1.605442 & -2.427605\end{array}$

C $4.903007 \quad-2.2255341 .100463$

H $4.434757 \quad-0.2554730 .411947$

C $3.322194 \quad-0.6218632 .204913$

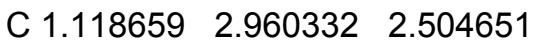

C $-0.0935913 .793542 \quad-2.366409$

H $-1.001633 .079578-0.573203$ 
C $-0.070598 \quad 4.948019-0.114558$

C $3.319573 .64925-1.607377$

H 2.5696124 .290350 .300771

C $3.9284692 .658308 \quad 0.609358$

C -3.660989 $-0.554747 \quad 0.769594$

$\mathrm{H}-2.424917-0.0223692 .242644$

C - $0.547276-3.0343381 .903566$

C $-0.229202 \quad 0.668715 \quad 3.2355$

C -2.062042 $0.960342 \quad-3.002867$

H $-2.440662 \quad 1.389534 \quad-0.954662$

H $0.061225-1.413735-4.195604$

C $-1.3083880 .203252 \quad-3.927626$

H 2.77709 -4.230128 0.392973

C $1.123445 \quad-4.742438 \quad 1.637148$

H $1.93136-2.420366-3.307668$

H $1.684787-3.765086-2.182187$

H $3.018576-3.808978-3.337328$

H $4.951605-2.213836-3.164304$

H $5.161598-1.170224-1.757119$

H $3.927575-0.785398-2.961422$

H $5.659701-1.8808411 .813866$

H $5.431176-2.5307770 .195682$

H $4.429259-3.1069521 .538998$

H $2.837584-1.4473552 .732769$

H $2.588606 \quad 0.174104 \quad 2.088731$

H $4.131017-0.2491052 .841847$

H $1.661925 \quad 3.858808 \quad 2.238474$

C $0.855683 \quad 2.722802 \quad 3.851373$

H $-1.044898 \quad 4.244045 \quad-2.668235$

H $0.011619 \quad 2.849377 \quad-2.902365$

H $0.6989194 .468385-2.696732$

H $0.824153 \quad 5.533351 \quad-0.345772$

$\mathrm{H}-0.1382654 .836544 \quad 0.968977$

H $-0.933195 \quad 5.541557 \quad-0.437313$

H $4.2873914 .157974 \quad-1.549197$

H $2.644664 \quad 4.293994 \quad-2.169013$

H $3.461164 \quad 2.727674 \quad-2.180321$

H $4.2747 \quad 1.762045 \quad 0.091692$

H $3.624292 .3745 \quad 1.616954$

H $4.7820493 .339135 \quad 0.695137$

C $-4.079263-1.578089-0.108568$

C $-4.568280 .484605 \quad 1.090467$

C $-0.070229-4.3080952 .203253$

H - 1.497753 -2.718495 2.31804

C $0.187587 \quad 1.560079 \quad 4.220764$

H $-0.730822 \quad-0.2393893 .557293$

$\mathrm{H}-2.799301 \quad 1.671365 \quad-3.359129$

$\mathrm{H}-1.4526120 .352332 \quad-4.992635$

H $1.497478 \quad-5.741377 \quad 1.834482$

H $1.183586 \quad 3.433533 \quad 4.602112$

C -5.35659-1.508485 -0.672862

C $-3.211714-2.770995-0.422068$

C $-5.8401720 .496998 \quad 0.516663$

C -4.176265 1.582372 .05105

$\mathrm{H}-0.637422-4.9606122 .858532$

$\mathrm{H}-0.0033461 .342473 \quad 5.266033$

H -5.661241 -2.302024 -1.349733
C -6.256261 $-0.482767-0.385167$

H - $2.319073-2.499323-0.989685$

H -6.520359 $1.303255 \quad 0.777689$

$\mathrm{H}-3.2571822 .089461 \quad 1.739281$

C $-7.617593-0.425085-1.037287$

H -7.998109 $-1.426507-1.254985$

H - $2.874081 \quad-3.2663310 .490709$

H -3.769653 $-3.497963-1.015886$

$\begin{array}{llll}\mathrm{H}-8.345529 & 0.080334 & -0.397351\end{array}$

H -7.582303 $0.122083 \quad-1.986483$

H -3.999508 $1.202725 \quad 3.065687$

H $-4.9670122 .331028 \quad 2.122999$

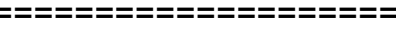

IS2-2

$\mathrm{Ni}-1.415872-0.226266-0.07491$

$\begin{array}{llll}\mathrm{P}-1.669035 & 1.786849 & -1.075063\end{array}$

P $-1.088655 \quad-0.3320782 .130536$

Si1.486735 0.8397350 .061396

C -2.628657 $-1.502674-0.607793$

C $0.940369-0.818504-0.644421$

C $0.284056-0.921618-1.890771$

C $-0.1197522 .60991-1.722396$

C - $2.8021881 .742484 \quad-2.602068$

C - $2.452466 \quad 3.242244 \quad-0.106228$

$\begin{array}{lll}\text { C } 0.128262 & 0.942761 \quad 2.753148\end{array}$

C $-0.311555-1.9110582 .882085$

C -2.603367 $-0.059963 \quad 3.240386$

N $3.272937 \quad 0.687858 \quad 0.228992$

C $1.1364592 .283039-1.152734$

C $1.113271 .425722 \quad 1.859712$

$\mathrm{N}-3.402824-2.340471-0.918322$

C $1.434339-2.025125-0.085181$

C $0.121209-2.161406-2.53023$

H $0.024096-0.027749-2.43629$

C - $0.1896593 .62348-2.690752$

C - $2.269897 \quad 0.814832 \quad-3.700969$

$\mathrm{H}-2.8502542 .755026 \quad-3.011446$

C $-4.2239721 .325437 \quad-2.195118$

C $-3.041447 \quad 4.404134 \quad-0.920958$

H -3.274322 $2.752128 \quad 0.42377$

C -1.479644 $3.804609 \quad 0.938634$

C $0.044164 \quad 1.488738 \quad 4.042891$

C $-0.877454-3.2042862 .275815$

H $0.722189-1.8182552 .538714$

C $-0.276541-1.9928164 .414298$

C -3.663195 -1.1505893 .015539$

H -2.263763 -0.1326764 .27755$

C -3.244032 $1.319204 \quad 3.03964$

C $4.203786-0.29406-0.198249$

H 3.5497611 .0268591 .139749

C $2.245607 \quad 3.067063 \quad-1.518656$

C $1.9402112 .476692 \quad 2.304615$

C - $4.29473-3.423744-1.247399$

C $1.268065-3.247706-0.713629$

H $2.002049-1.9923840 .837093$

H - $0.369673-2.200134-3.496817$ 
C $0.601944-3.321222-1.945756$ $\mathrm{H}-1.134323 .853448-3.166355$

$\begin{array}{llll}\text { C } 0.931068 & 4.364037 & -3.056945\end{array}$ H -2.212088 -0.21623-3.346305

$\mathrm{H}-1.280333 \quad 1.116502 \quad-4.053358$

$\mathrm{H}-2.946364 \quad 0.837122 \quad-4.562511$

H $-4.8736391 .311479 \quad-3.077155$

H -4.671426 $2.007421 \quad-1.468529$

H -4.221826 $0.325273-1.757966$

H -3.509724 $5.117774 \quad-0.233769$

$\mathrm{H}-3.8074124 .093216-1.632388$

$\mathrm{H}-2.265158 \quad 4.947115 \quad-1.465134$

H $-0.666981 \quad 4.355136 \quad 0.457291$

$\mathrm{H}-1.0336983 .028271 .556803$

$\mathrm{H}-2.0082964 .502028 \quad 1.597044$

H $-0.6999041 .123036 \quad 4.738971$

C $0.878711 \quad 2.523215 \quad 4.459141$

H $-0.215945-4.0439912 .515992$

$\mathrm{H}-0.963203-3.136681 .191909$

$\mathrm{H}-1.86281-3.4444312 .681156$

$\mathrm{H}-1.277266-2.1063824 .840695$

H $0.193514-1.1185134 .868416$

H $0.303406-2.8708034 .720423$

$\mathrm{H}-4.553875-0.9212483 .610378$

H -3.318055 -2.140874 3.313137

H -3.960532 -1.1961041 .965264$

$\mathrm{H}-3.666958 \quad 1.403409 \quad 2.036328$

H -2.539429 2.138682 3.183394

H -4.062037 $1.450777 \quad 3.756375$

C $4.443095-0.579422-1.560639$

$\begin{array}{lll}\text { C } 4.964339 & -0.975922 & 0.783868\end{array}$

$\begin{array}{llll}C & 2.152727 & 4.101866 & -2.445302\end{array}$

H $3.2094962 .839999-1.078566$

C $1.827318 \quad 3.029807 \quad 3.577714$

H $2.6776982 .906496 \quad 1.633572$
C -4.296488 $-3.608058-2.776142$

C $-3.791851-4.702473-0.55204$

C $-5.705848-3.059931-0.749604$

H $1.678144-4.145098-0.263176$

H $0.489159-4.276293-2.447773$

H $0.8440395 .146258 \quad-3.803648$

H 0.7753652 .9360895 .456863

C $5.381199-1.560667-1.895514$

C $3.7662090 .179481-2.674483$

C $5.904671-1.931546 \quad 0.396449$

$\begin{array}{llll}\text { C } 4.774561 & -0.679118 & 2.252708\end{array}$

H $3.032164 \quad 4.683415 \quad-2.701028$

H $2.4696553 .853167 \quad 3.870859$

H -4.639712 $-2.698444-3.273553$

H $-4.965195-4.427807-3.05075$

H -3.292705 $-3.842657-3.136101$

H - $2.781367-4.948695-0.884339$

H - 4.452762 -5.53939-0.792011

H $-3.776589-4.5710880 .531818$

H $-5.706266-2.9086690 .331885$

$\mathrm{H}-6.40211-3.86804-0.987414$

H -6.060073 - $2.144695-1.228671$

H $5.548819-1.769201-2.948694$

$\begin{array}{llll}\text { C } 6.122547 & -2.257472 & -0.942106\end{array}$

H $2.682423 \quad 0.053746 \quad-2.671704$

H $6.475852 \quad-2.4405011 .168274$

H $3.732718-0.8052292 .565768$

$\begin{array}{lll}\text { C } 7.105356 & -3.3327 & -1.34178\end{array}$

H $7.5444 \quad-3.12696-2.321557$

H $7.920644-3.417908-0.618533$

H $6.620531-4.313995-1.402919$

H $5.391597 \quad-1.3421312 .861645$

H $5.058883 \quad 0.3494292 .509127$

H $3.9652621 .250886-2.600085$

H $4.134092-0.165092-3.643247$ 


\section{References}

(1) Herbert, D. E.; Lara, N. C.; Agapie, T. Chem. Eur. J. 2013, 19, 16453-16460.

(2) Braunschweig, H.; Gross, M.; Radacki, K. Organometallics 2007, 26, 6688-6690.

(3) Frisch, M. J.; Trucks, G. W.; Schlegel, H. B.; Scuseria, G. E.; Robb, M. A.; Cheeseman, J. R.; Scalmani, G.; Barone, V.; Mennucci, B.; Petersson, G. A.; Nakatsuji, H.; Caricato, M.; Li, X.; Hratchian, H. P.; Izmaylov, A. F.; Bloino, J.; Zheng, G.; Sonnenberg, J. L.; Hada, M.; Ehara, M.; Toyota, K.; Fukuda, R.; Hasegawa, J.; Ishida, M.; Nakajima, T.; Honda, Y.; Kitao, O.; Nakai, H.; Vreven, T.; Montgomery, Jr., J. A.; Peralta, J. E.; Ogliaro, F.; Bearpark, M.; Heyd, J. J.; Brothers, E.; Kudin, K. N.; Staroverov, V. N.; Keith, T.; Kobayashi, R.; Normand, J.; Raghavachari, K.; Rendell, A.; Burant, J. C.; Iyengar, S. S.; Tomasi, J.; Cossi, M.; Rega, N.; Millam, J. M.; Klene, M.; Knox, J. E.; Cross, J. B.; Bakken, V.; Adamo, C.; Jaramillo, J.; Gomperts, R.; Stratmann, R. E.; Yazyev, O.; Austin, A. J.; Cammi, R.; Pomelli, C.; Ochterski, J. W.; Martin, R. L.; Morokuma, K.; Zakrzewski, V. G.; Voth, G. A.; Salvador, P.; Dannenberg, J. J.; Dapprich, S.; Daniels, A. D.; Farkas, O.; Foresman, J. B.; Ortiz, J. V.; Cioslowski, J.; Fox, D. J. Gaussian 09, Revision D.01; Gaussian, Inc.: Wallingford CT, 2013.

(4) Becke, A. D. Density-Functional Thermochemistry. III. The Role of Exact Exchange. J. Chem. Phys. 1993, $98,5648-5652$.

(5) Lee, C.; Yang, W.; Parr, R. G. Development of the Colic-Salvetti Correlation-Energy Formula into a Functional of the Electron Density. Phys. Rev. B 1988, 37, 785-789.

(6) Vosko, S. H.; Wilk, L.; Nusair, M. Accurate Spin-Dependent Electron Liquid Correlation Energies for Local Spin Density Calculations: A Critical Analysis. Can. J. Phys. 1980, 58, 1200-1211. 